\title{
A phylogenetic revision of Penicillium sect. Exilicaulis, including nine new species from fynbos in South Africa
}

\author{
Cobus M. Visagie ${ }^{1,2,3}$, Keith A. Seifert ${ }^{1}$, Jos Houbraken², Robert A. Samson², and Karin Jacobs ${ }^{3}$
}

1Biodiversity (Mycology), Agriculture and Agri-Food Canada, Ottawa, ON K1A0C6, Canada; corresponding author e-mail: cobusvisagie9@gmail. com

${ }^{2}$ CBS-KNAW Fungal Biodiversity Centre, Uppsalalaan 8, NL-3584 CT Utrecht, The Netherlands

${ }^{3}$ Department of Microbiology, University of Stellenbosch, Private Bag X1, Stellenbosch 7600, South Africa

\begin{abstract}
A survey of the fynbos biome in South Africa resulted in the isolation of 61 Penicillium species from Protea repens infructescences, air, and soil samples. Fourteen of these belong to Penicillium sect. Exilicaulis and therefore we considered it an opportunity to re-evaluate the taxonomy of the section. Phylogenetic comparisons of the ITS, $\beta$-tubulin, calmodulin and RPB2 gene regions of the 76 section Exilicaulis species, revealed 52 distinct species, including nine new species from fynbos. Morphological comparisons confirmed the novelty for most of these, however, new species closely related to $P$. rubefaciens did not show significant or consistent morphological differences and we thus placed a bias on phylogenetic data applying the Genealogical Concordance Phylogenetic Species Recognition (GCPSR) concept. In this paper we describe the nine new species and update the accepted species list and resolve synonyms in the section. Importantly, we reveal that $P$. citreosulfuratum is the correct name for the clade previously considered to represent $P$. toxicarium fide Serra et al. (2008).

The nine new species are: Penicillium atrolazulinum, P. consobrinum, P. cravenianum, P. hemitrachum, P. pagulum, $P$. repensicola, P. momoii, P. subturcoseum, and $P$. xanthomelinii spp. nov.
\end{abstract}

Article info: Submitted: 12 November 2015; Accepted: 15 May 2016; Published: 1 June 2016.

\section{INTRODUCTION}

Pitt (1980) introduced the name Penicillium sect. Exilicaulis, typified by $P$. restrictum, for species with monoverticillate conidiophores and non-vesiculated stipes. In the recent re-classification of Penicillium based on phylogenetic data (Houbraken \& Samson 2011), section Exilicaulis was redefined to include biverticillate species such as $P$. corylophilum, $P$. melinii, and $P$. raciborskii. With a limited strain and species sampling, Houbraken \& Samson (2011) separated species into two main clades. This was also supported by a more comprehensive RPB2 (RNA polymerase II second largest subunit) phylogeny revealing a well-supported main $P$. corylophilum clade (containing biverticillate species), whereas monoverticillate species resolved in several basal clades.

Section Exilicaulis has received some taxonomic attention in recent years, including the introduction of new species (Langlois et al. 2014, Peterson et al. 1999, 2011), but more importantly species and their metabolites were shown to be toxic and to affect human health (Kremer et al. 1989, Lyratzopoulos et al. 2002, McMullin et al. 2014, Miyake 1940, Ohnishi et al. 2002, Peterson et al. 1999, Rosa et al. 2010, Unoura et al. 2011, Yoshida et al. 1992). Serra et al. (2008) studied the diversity of Penicillium species in cork bark with a focus on some species of sect. Exilicaulis, stressing the importance of applying the Genealogical Concordance Phylogenetic Species Recognition (GCPSR) concept (Taylor et al. 2000) to Penicillium. This approach to species delineation has become standard in Penicillium, especially in studies focused on resolving the taxonomy of specific sections (Houbraken \& Samson 2011, Houbraken et al. 2011, 2014, Peterson et al. 2011, Rivera \& Seifert 2011, Visagie et al. 2013). This concept was also applied in section Exilicaulis, but the focus was mostly on a subset of species from the P. parvum-clade (Langlois et al. 2014, Peterson et al. 1999, 2011) and the $P$. citreonigrum complex (Serra et al. 2008), but did not include all species belonging to these clades.

During a survey of species diversity in the fynbos biome, situated in the Western Cape of South Africa, Penicillium was one of the dominant genera isolated, with roughly 61 species found. Fourteen belong to section Exilicaulis and nine of those represent new species. Here we compare the morphology of these new species with all others previously classified in section Exilicaulis and apply GCPSR using phylogenies of the rDNA region (ITS), $\beta$-tubulin (BenA), calmodulin (CaM), and RPB2 genes. This paper follows previous papers reporting new Penicillium species isolated during this survey belonging to sections Aspergilloides (Houbraken et al. 2014), Citrina (Visagie et al. 2014c), Lanata-Divaricata (Visagie et al. 2015), Sclerotiora (Visagie et al. 2013), and Torulomyces (Visagie et al. 2016a).

() 2016 International Mycological Association

You are free to share - to copy, distribute and transmit the work, under the following conditions:

Attribution: $\quad$ You must attribute the work in the manner specified by the author or licensor (but not in any way that suggests that they endorse you or your use of the work).

Non-commercial: $\quad$ You may not use this work for commercial purposes.

No derivative works: You may not alter, transform, or build upon this work.

For any reuse or distribution, you must make clear to others the license terms of this work, which can be found at http://creativecommons.org/licenses/by-nc-nd/3.0/legalcode. Any of the above conditions can be waived if you get permission from the copyright holder. Nothing in this license impairs or restricts the author's moral rights. 


\section{MATERIAL AND METHODS}

\section{Sampling and isolation}

Soil, air and Protea repens infructescence samples were taken

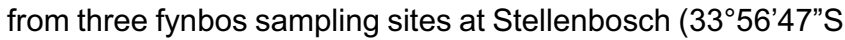

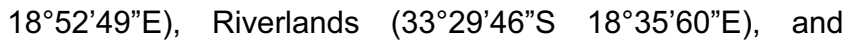
Struisbaai $\left(33^{\circ} 45^{\prime} 06^{\prime \prime} S 18^{\circ} 58^{\prime} 59^{\prime \prime E}\right)$. Potato dextrose agar (PDA) containing streptomycin (100 ppm), chloramphenicol $(50 \mathrm{ppm})$ and dichloran $(0.002 \mathrm{~g} / \mathrm{L})$ was used as isolation medium. Isolation plates were incubated at $25{ }^{\circ} \mathrm{C}$ for $7 \mathrm{~d}$, after which colonies resembling Penicillium were transferred to oatmeal agar (OA).

Isolations from soil were made using a dilution series by adding $5 \mathrm{~g}$ soil to $100 \mathrm{~mL} \mathrm{dH}_{2} \mathrm{O}$ and diluting this tenfold three times resulting in suspensions $10^{-1}, 10^{-2}$ and $10^{-3}$ of the original. In triplicate, $1 \mathrm{~mL}$ from each dilution was spread onto PDA plates. Isolations from Protea repens infructescences included sampling from bracts and mite populations. Firstly, infructescences were cut open and the mites shaken onto $1 \%$ water agar plates with chloroform used to temporarily sedate mites. Mites were transferred onto PDA plates using a fine needle and incubated on a lab bench $\left( \pm 21^{\circ} \mathrm{C}\right)$ on soapy water traps to prevent cross contamination by awaken mites roaming on plates. Roughly 15 bracts from infructescences were suspended in $100 \mathrm{~mL} \mathrm{dH}_{2} \mathrm{O}$. This solution was diluted tenfold six times resulting in suspensions $10^{-4}, 10^{-5}$ and $10^{-6}$ of the original and spread plates prepared similarly to the soil spread plates. Air was sampled close to the same sampled Protea repens bushes using a MAS-Eco ${ }^{\circledR}$ air sampler set to a volume of $50 \mathrm{~L}$.

\section{Reference strains}

Reference strains used for this study (Table 1), were obtained from the public collection of CBS (CBS-KNAW Fungal Biodiversity Centre, Utrecht) and cultures from the working collection of the Applied and Industrial Mycology group at the same institute (DTO). Strains isolated during this study are kept in a private collection at the department of Microbiology, Stellenbosch University, South Africa (CV). These strains were also deposited in DTO, with a subset of strains deposited into DAOMC (Canadian Collection of Fungal Cultures, Ottawa) and CBS.

\section{DNA extraction, sequencing and phylogenetic analysis}

DNA was extracted from strains grown on MEA for $7 \mathrm{~d}$ using the ZR Fungal/Bacterial DNA kit (ZymoResearch, $\mathrm{CA}$ ), with extracts stored at $4{ }^{\circ} \mathrm{C}$. Amplification of the ITS region, partial $\beta$-tubulin (BenA), calmodulin (CaM), and RNA polymerase II second largest subunit (RPB2) were made using primers and amplification profiles described in Visagie et al. (2014b). PCR and sequencing reactions followed similar methods to Visagie et al. (2013). For some strains, the partial $R P B 2$ gene was sequenced using internal sequencing primers $R P B 2$ Int $388 F$ and $R P B 2$ Int$527 R$ as described in Houbraken \& Samson (2011). Sequence contigs were assembled using CodonCode Aligner v. 4.0.1 (CodonCode Centerville, MA, USA). Newly generated sequences were deposited in GenBank with accession numbers provided in Table 1 .
Fynbos strains isolated during this study were added to a sequence database that includes all ex-type strains of species belonging to section Exilicaulis. All data sets were aligned using MAFFT v. 7.164b (Katoh \& Standley 2013) implementing the L-INS-i option. For multigene phylogenies, aligned data sets for each gene or region were concatenated in SeaView v. 4.4.1 (Gouy et al. 2010).

Phylogenies were constructed using both Maximum Likelihood (ML) and Bayesian tree inference $(\mathrm{BI})$ analyses. $\mathrm{ML}$ analyses were done using MEGA v. 6.06 (Tamura et al. 2013). The most suitable model for each data set was selected based on the lowest Akaike information criterion (AIC) value in MEGA. $\mathrm{ML}$ analyses were run by calculating an initial Bio-NeighbourJoining tree, followed by a Heuristic search with the NearestNeighbour-Interchange (NNI) option. Bootstrap analysis with 1000 replicates was used to represent support for nodes. For $\mathrm{BI}$, the most suitable model for each data set was selected in MrModeltest v. 2.3 (Nylander et al. 2004) based on the lowest AIC value. Subsequent analyses were run in MrBayes v. 3.2.1 (Ronquist \& Huelsenbeck 2003) using three sets of four chains (one cold and three heated) and stopped when the average standard of deviation for split frequencies reached 0.01 . The sample frequency was then set at 100 and $25 \%$ of trees were removed as burn-in. ML phylograms were used for representing phylogenies obtained from $\mathrm{ML}$ and $\mathrm{BI}$ analyses, with bootstrap values ( $\geq 80 \%$ ) and/or posterior probabilities ( $\geq$ $0.95 \mathrm{pp}$ ) both indicated above thickened branches.

\section{Morphology}

Fynbos strains were characterised on Czapek yeast autolysate agar (CYA), Blakeslee's malt extract agar (MEA; Bacto), yeast extract sucrose agar (YES), $25 \%$ glycerol nitrate agar (G25N), and creatine sucrose agar (CREA), incubated at 25 ${ }^{\circ} \mathrm{C}$ for $7 \mathrm{~d}$, with additional CYA plates incubated at 30 and 37 ${ }^{\circ} \mathrm{C}$. CREA contains bromocresol purple and is used as a rapid method for detecting acid production by colonies, indicated by a colour change in the medium from purple to yellow (Frisvad 1981). Media preparation, inoculation technique, incubation conditions and microscope preparations were standardised according to the recommended methods published in Visagie et al. (2014b). Colour names and codes used in descriptions are based on Kornerup \& Wanscher (1967). An Olympus SZX12 dissecting microscope and Olympus BX50 light microscope equipped with an Evolution MP digital microscope camera and ImagePro v. 6.0 software were used for microphotography. Photographic plates were prepared in Adobe Photoshop CS6.

\section{RESULTS}

\section{Sampling and isolations}

Penicillium sect. Exilicaulis was the dominant group isolated from the fynbos, with 740 isolates accounting for roughly $43 \%$ of the total obtained. Strains belonging to the section were sorted into morpho-groups based on colony characters on CYA and MEA, with a subset of strains from each group subsequently sequenced and compared with reference sequences. The dominance of $P$. citreosulfuratum (197 isolates in total, with only three each from air and soil) and 


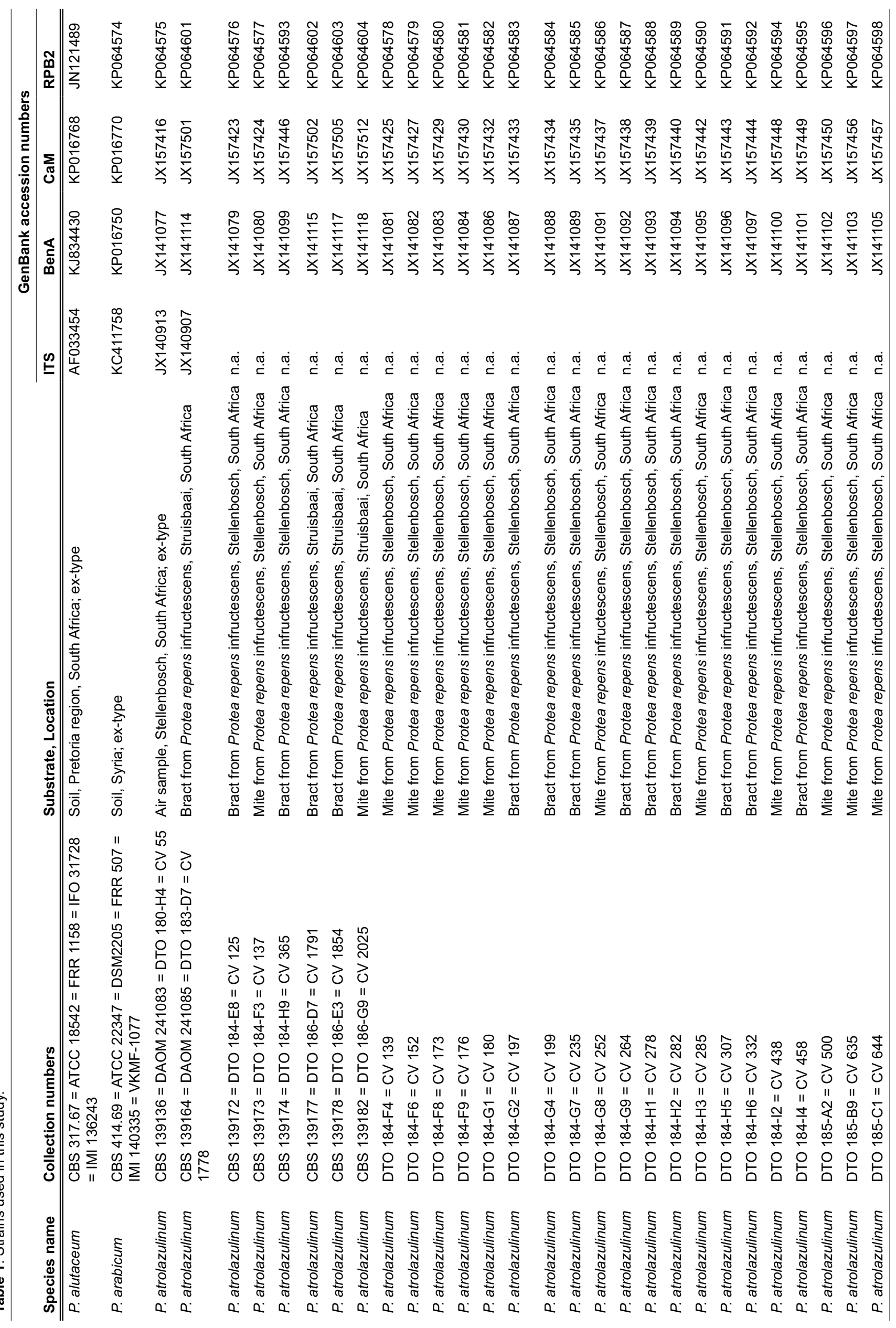




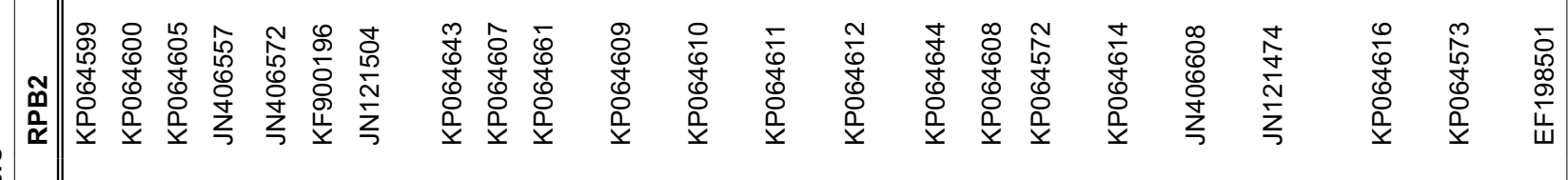
on

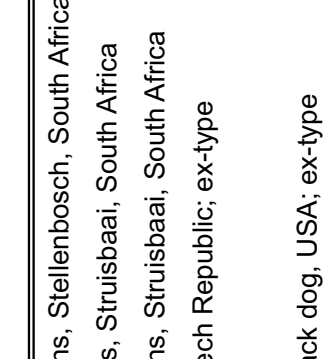

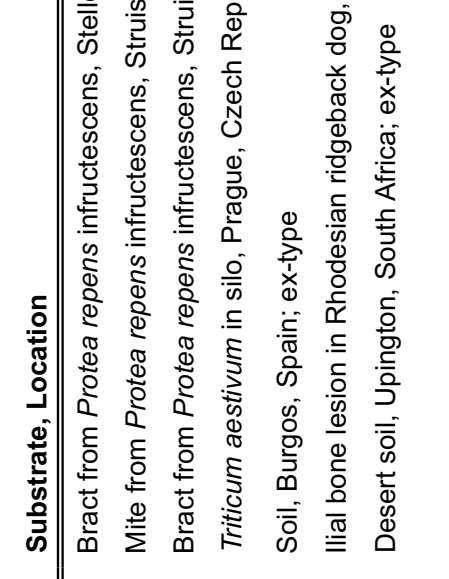

$\begin{array}{lllll} & & \\ 0\end{array}$

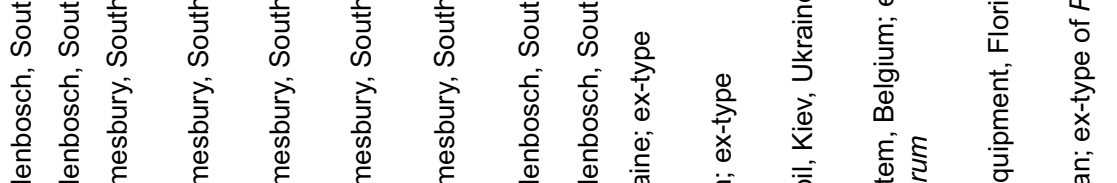

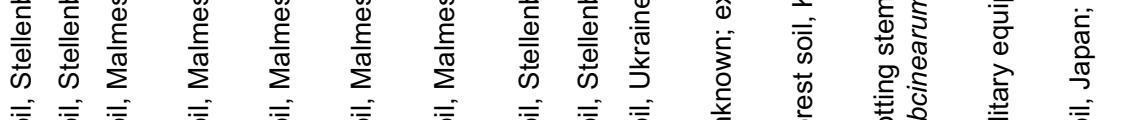

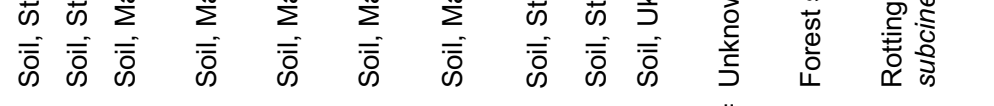

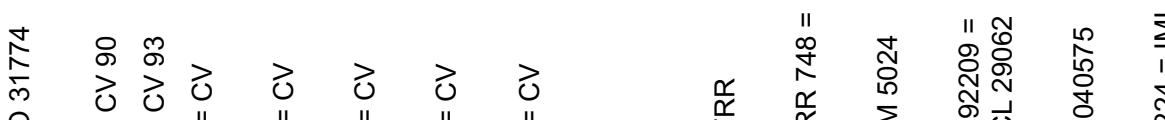

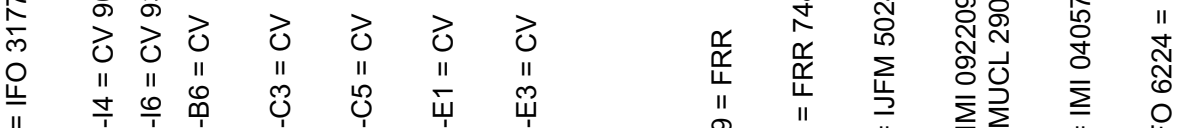

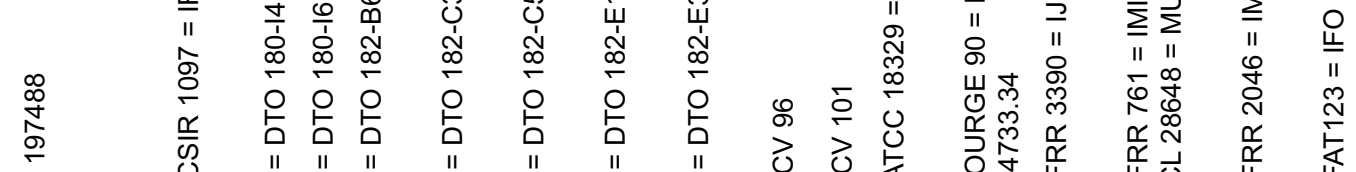
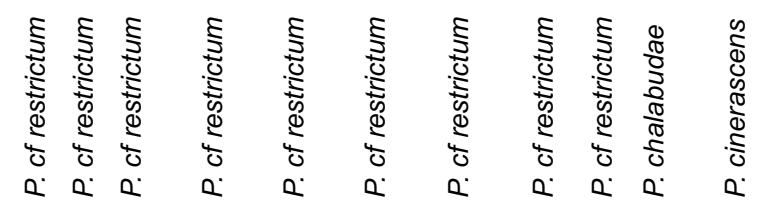


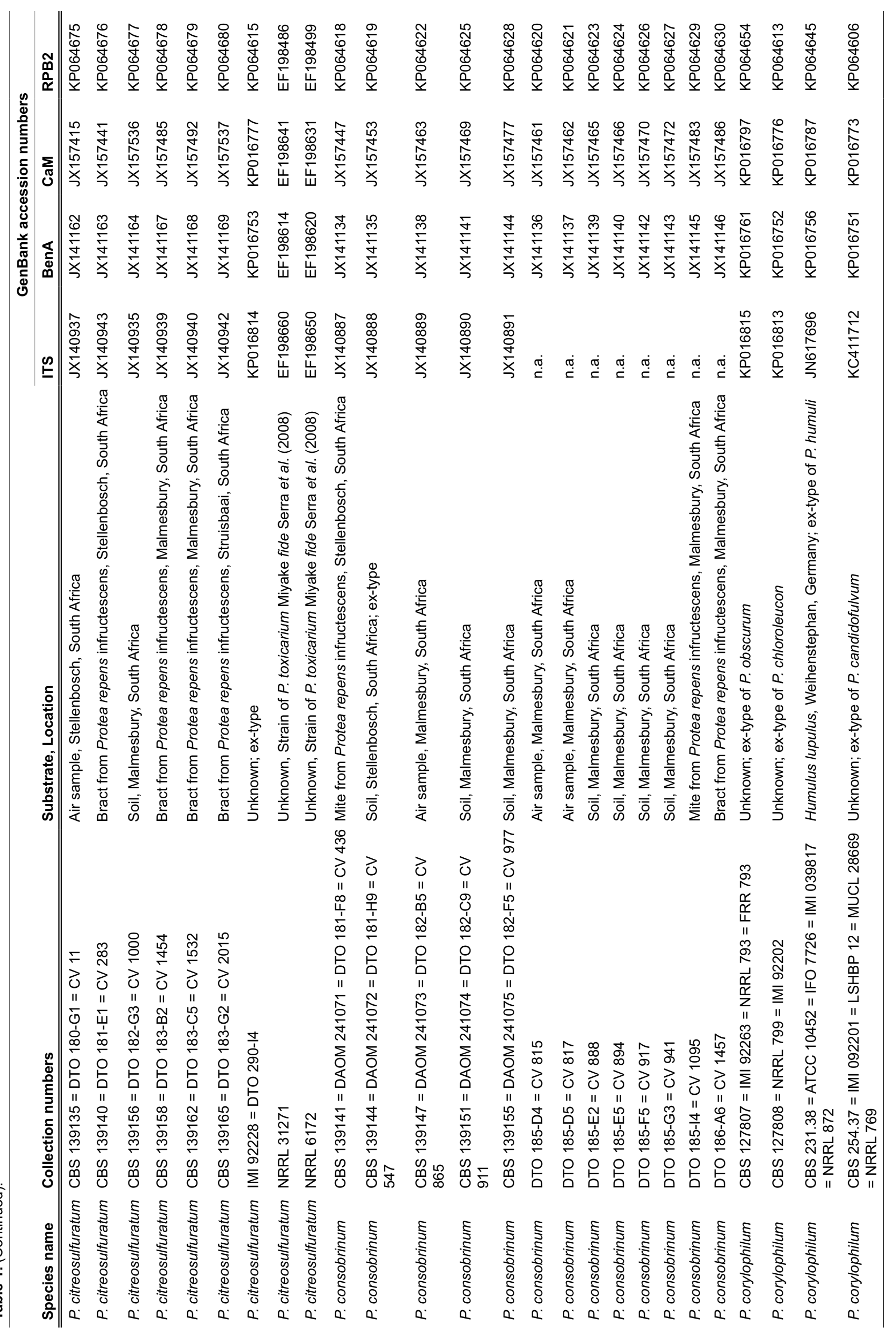




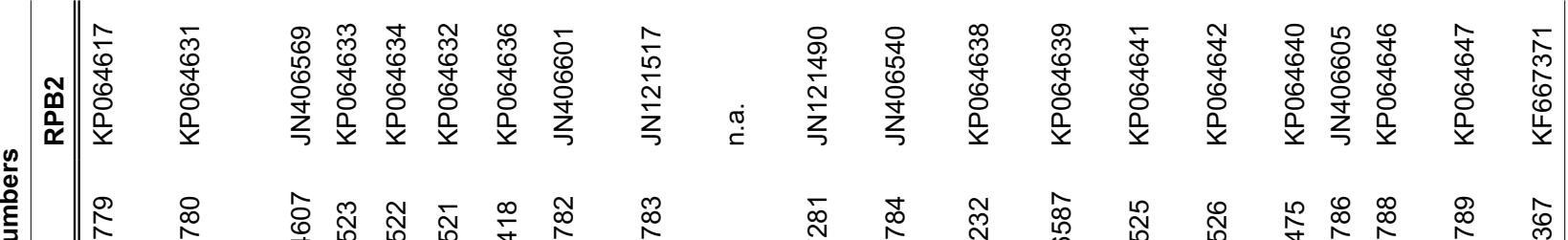

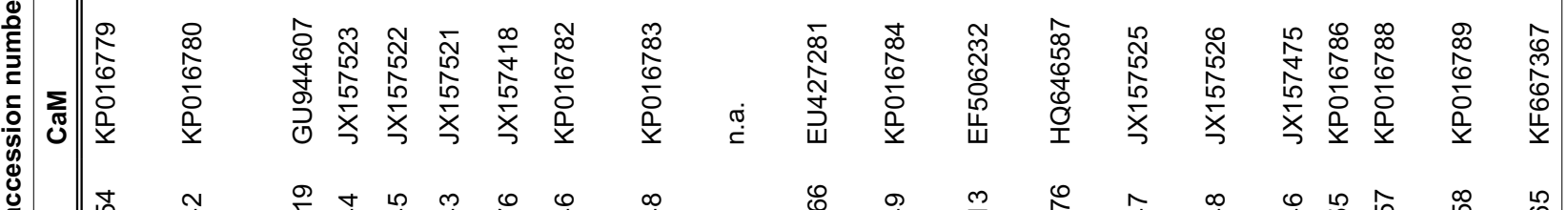

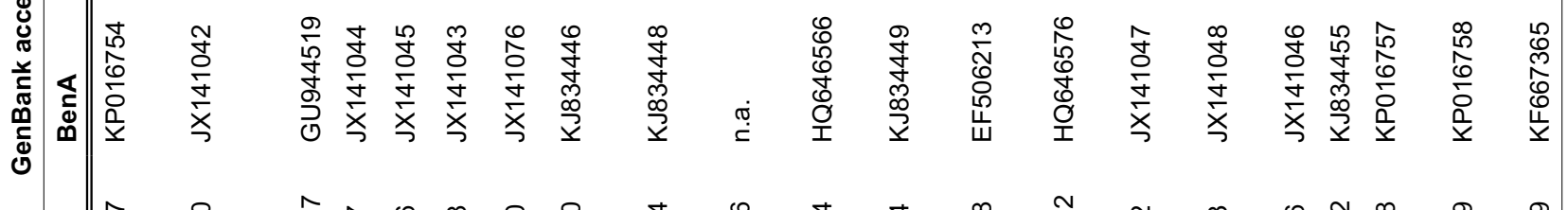

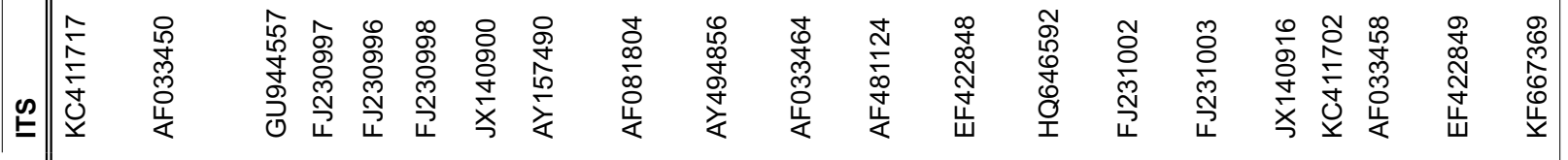

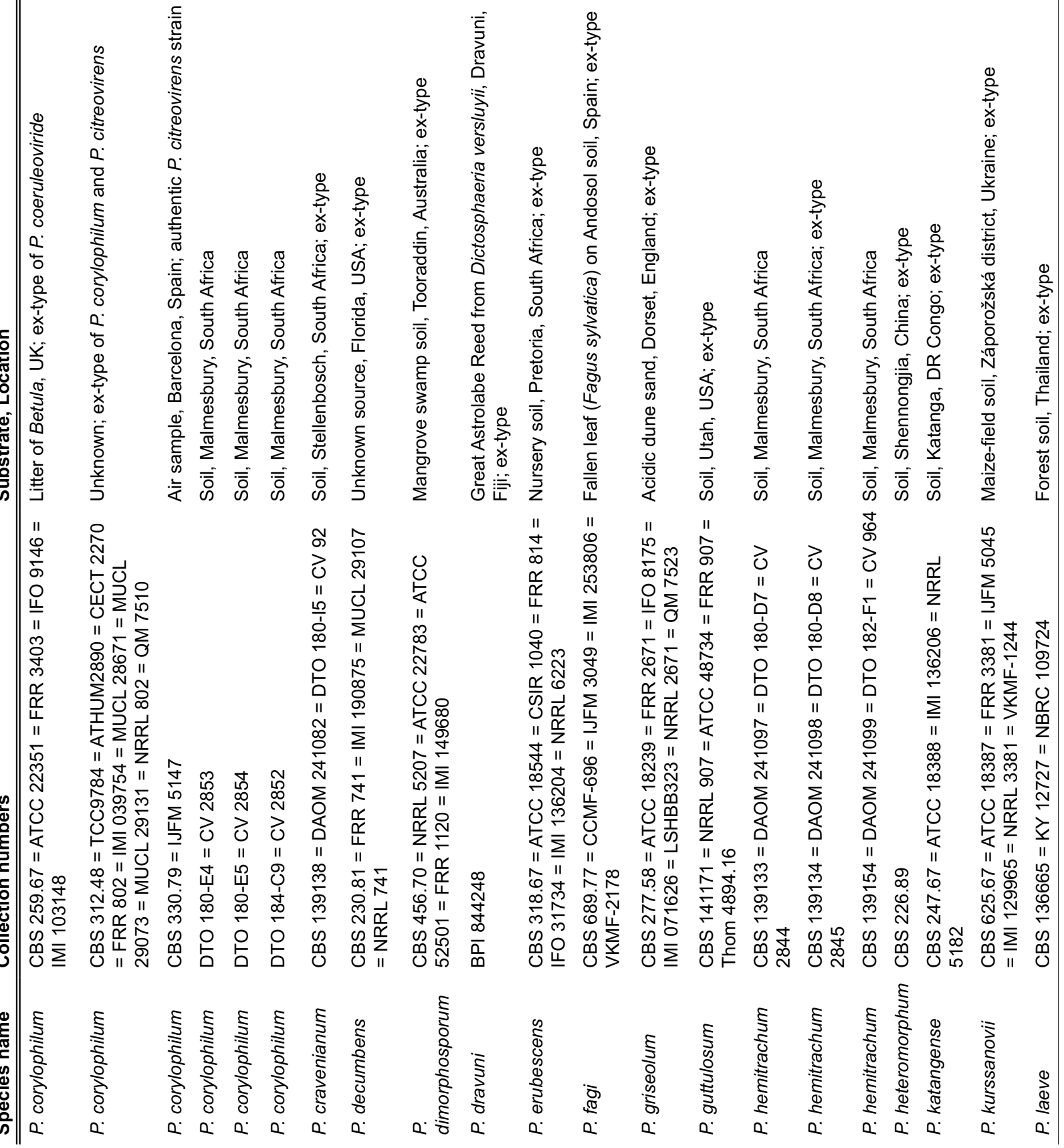




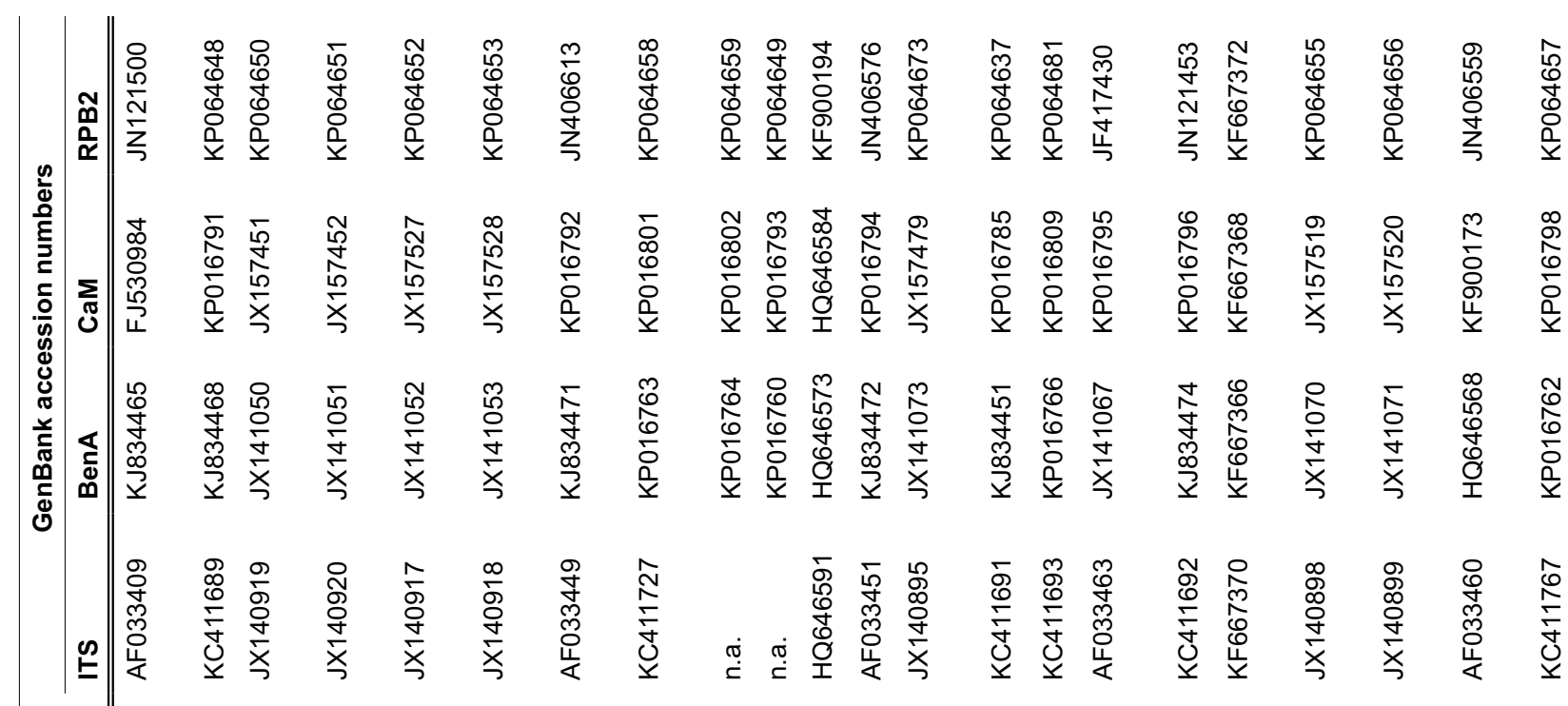

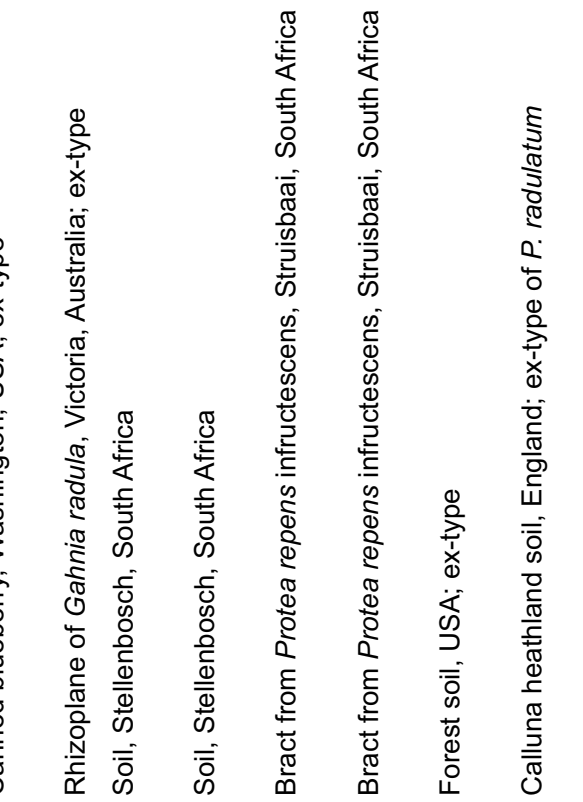

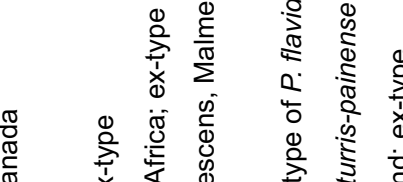

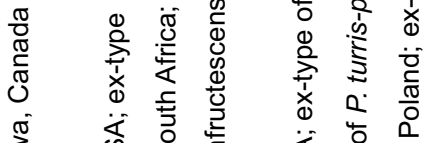

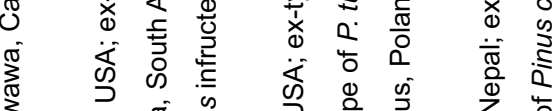<smiles>COC(=O)C(C)C</smiles>

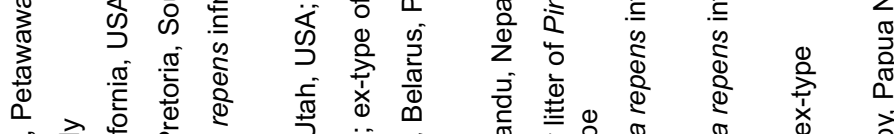

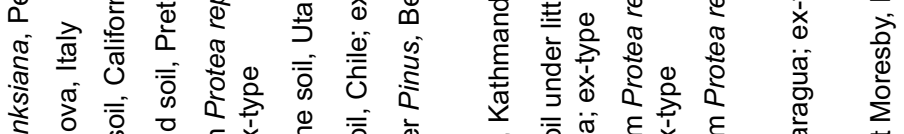

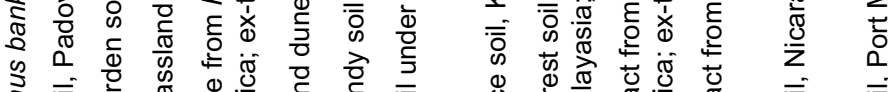
的

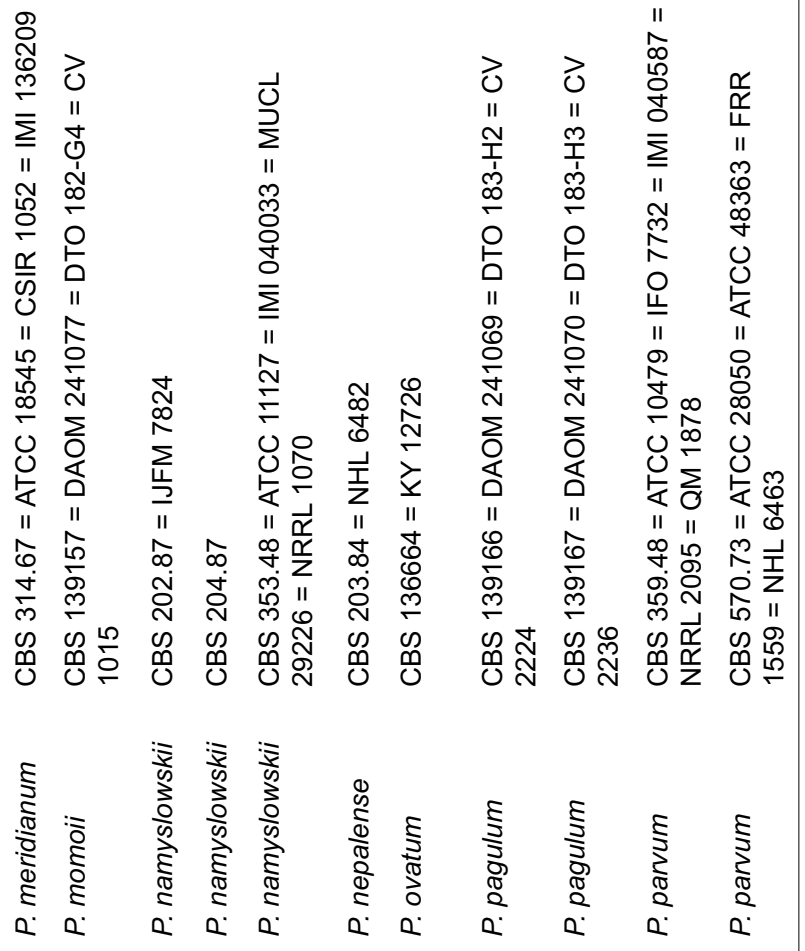

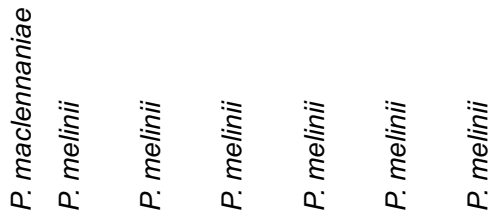

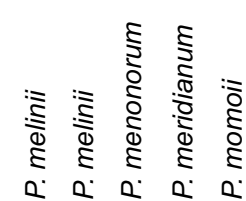




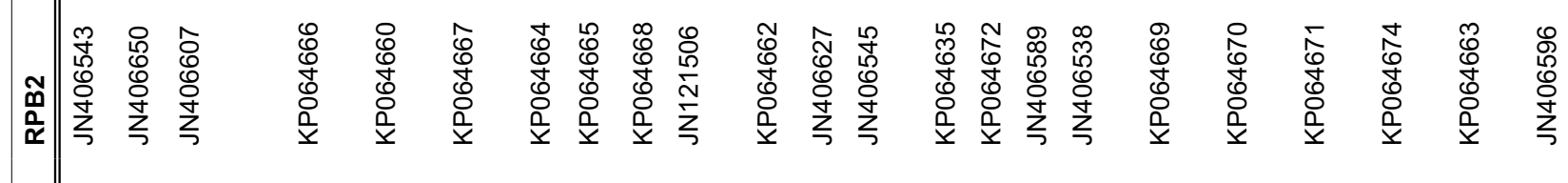

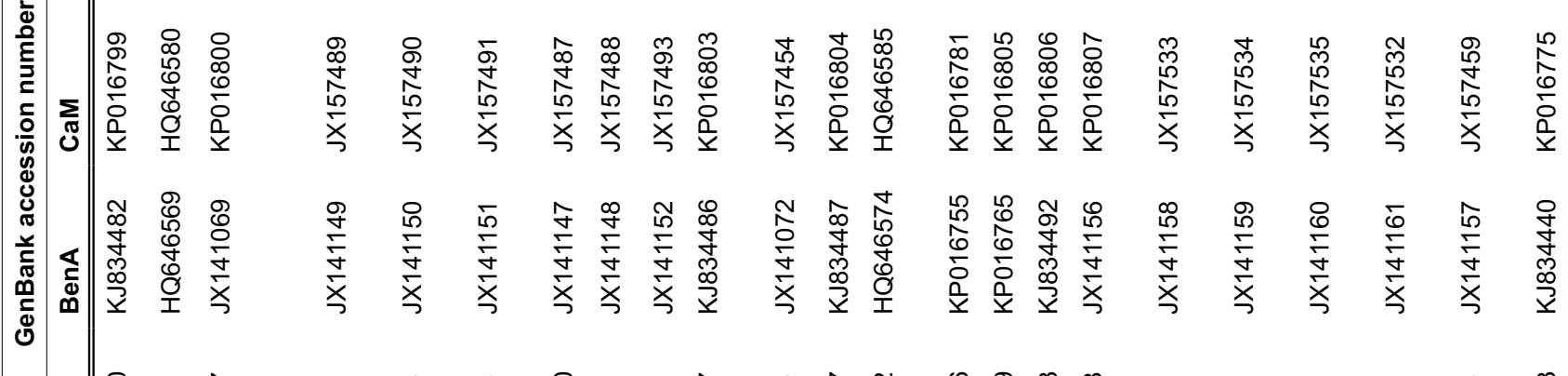

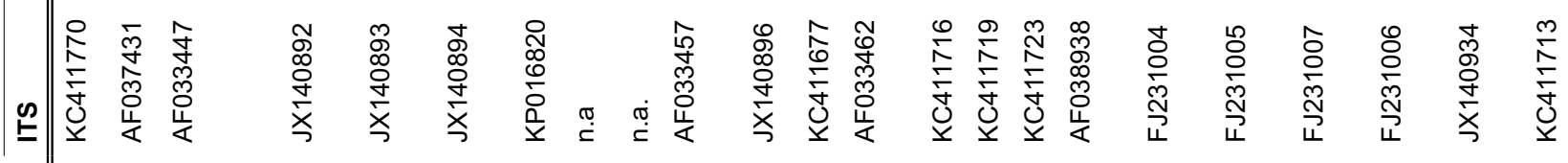

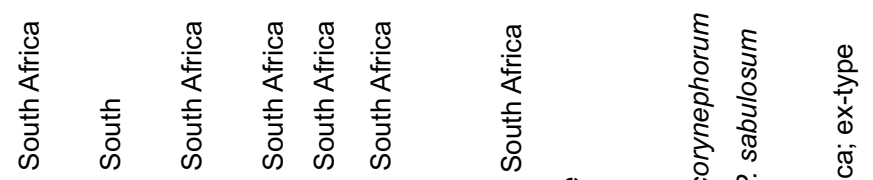

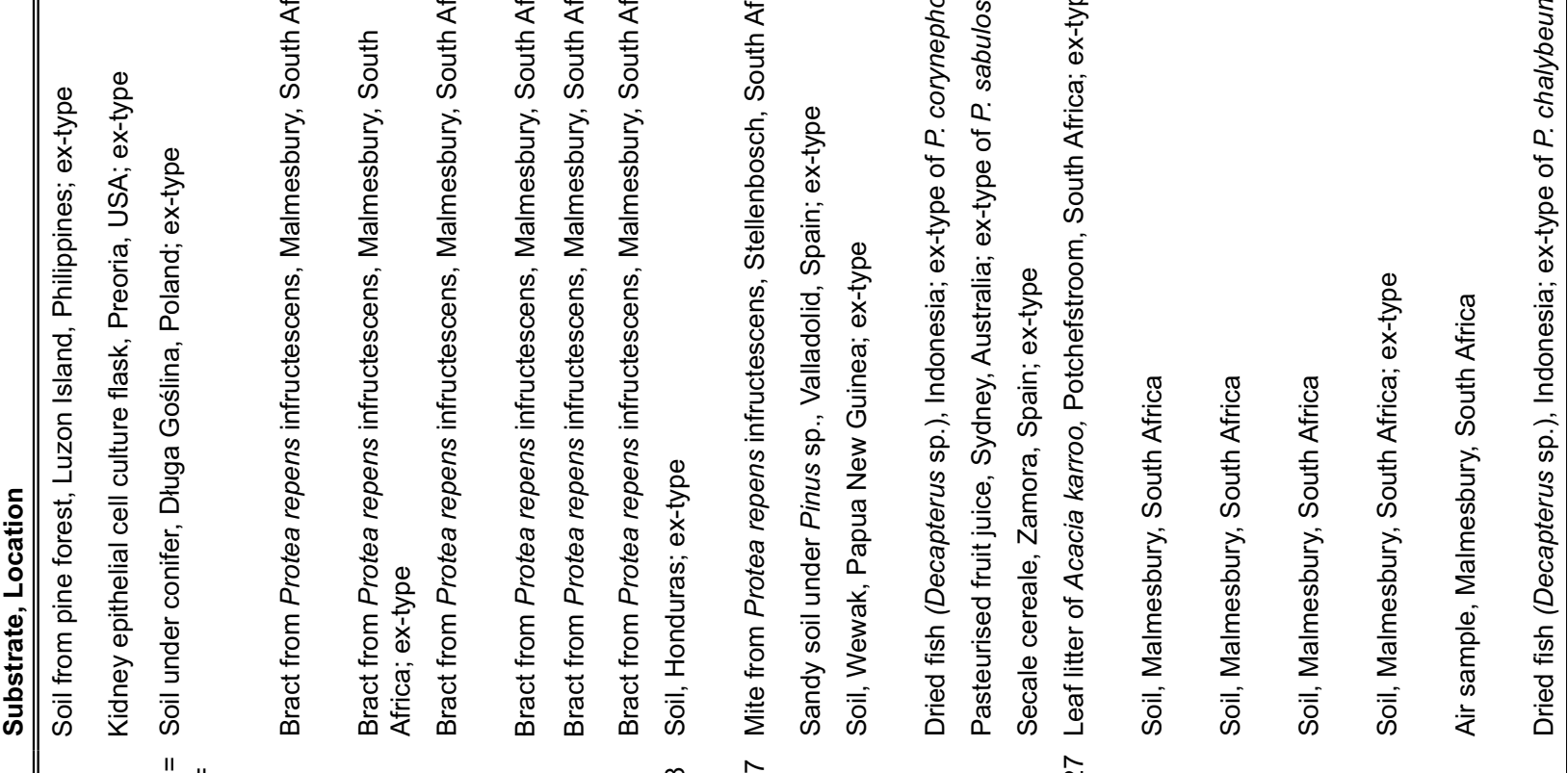

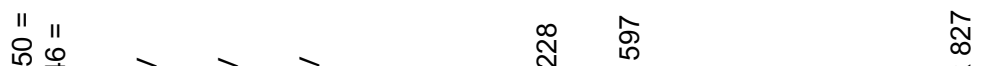

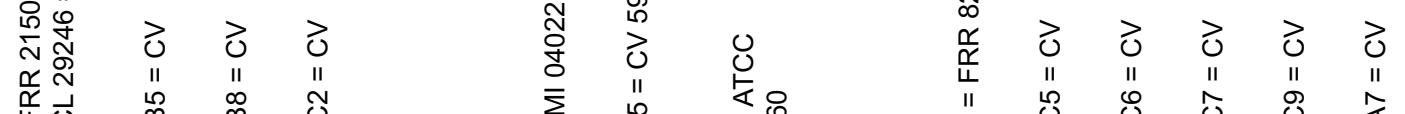

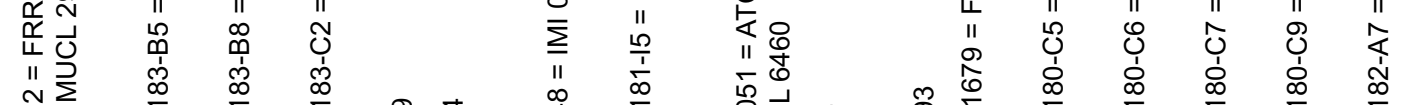

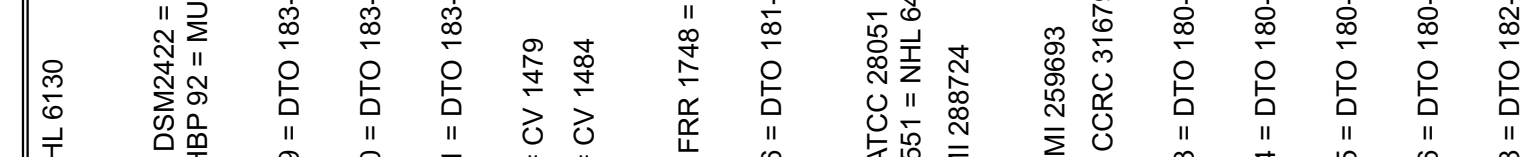

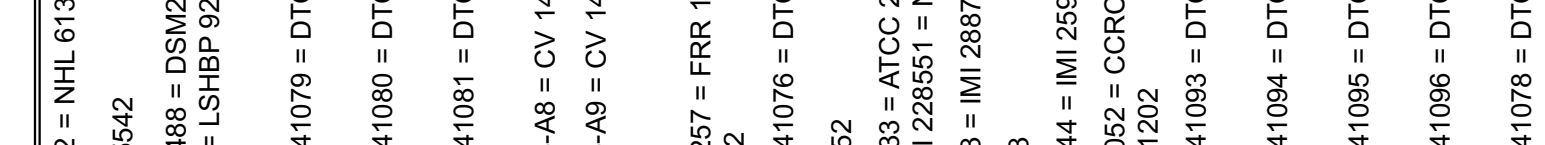

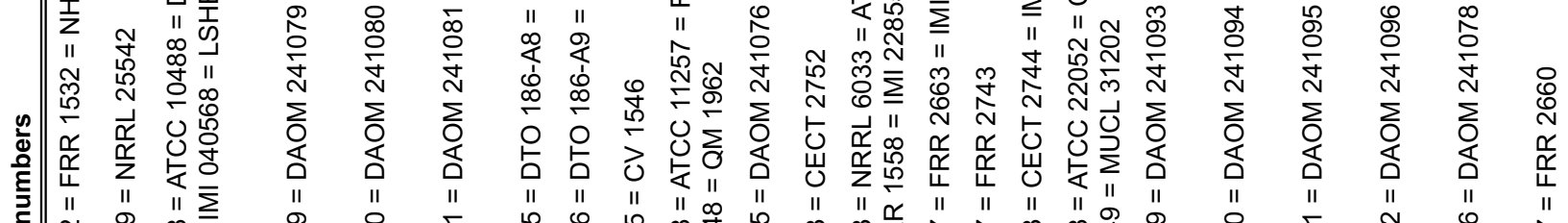

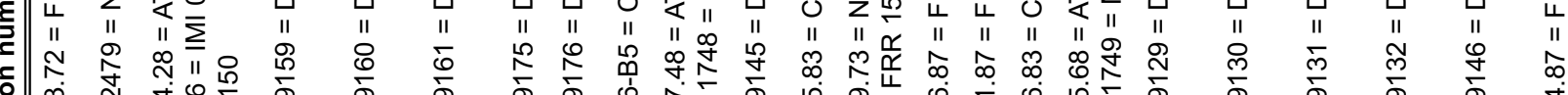

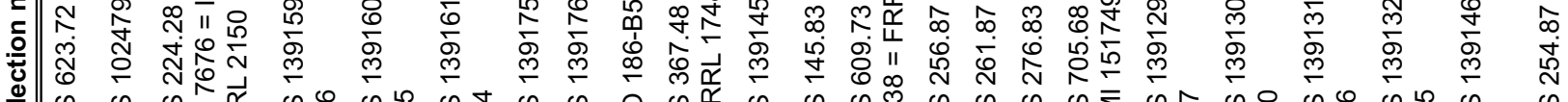

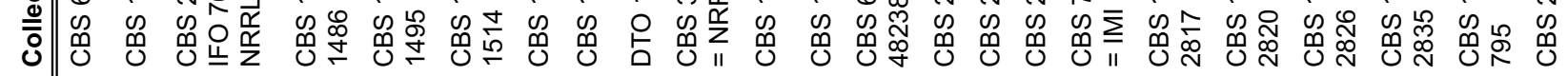
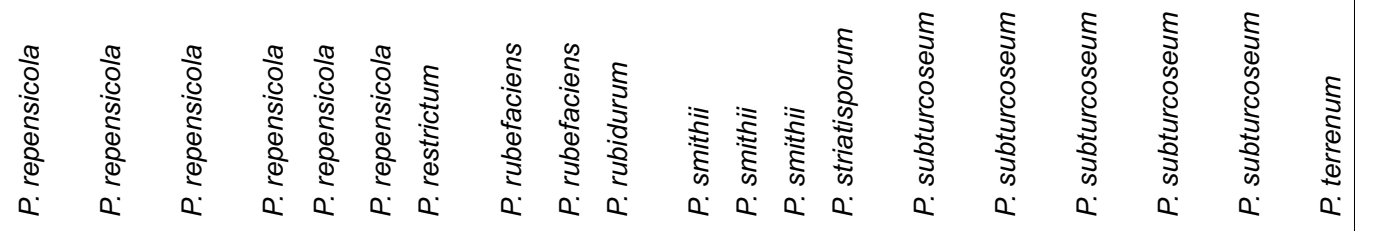


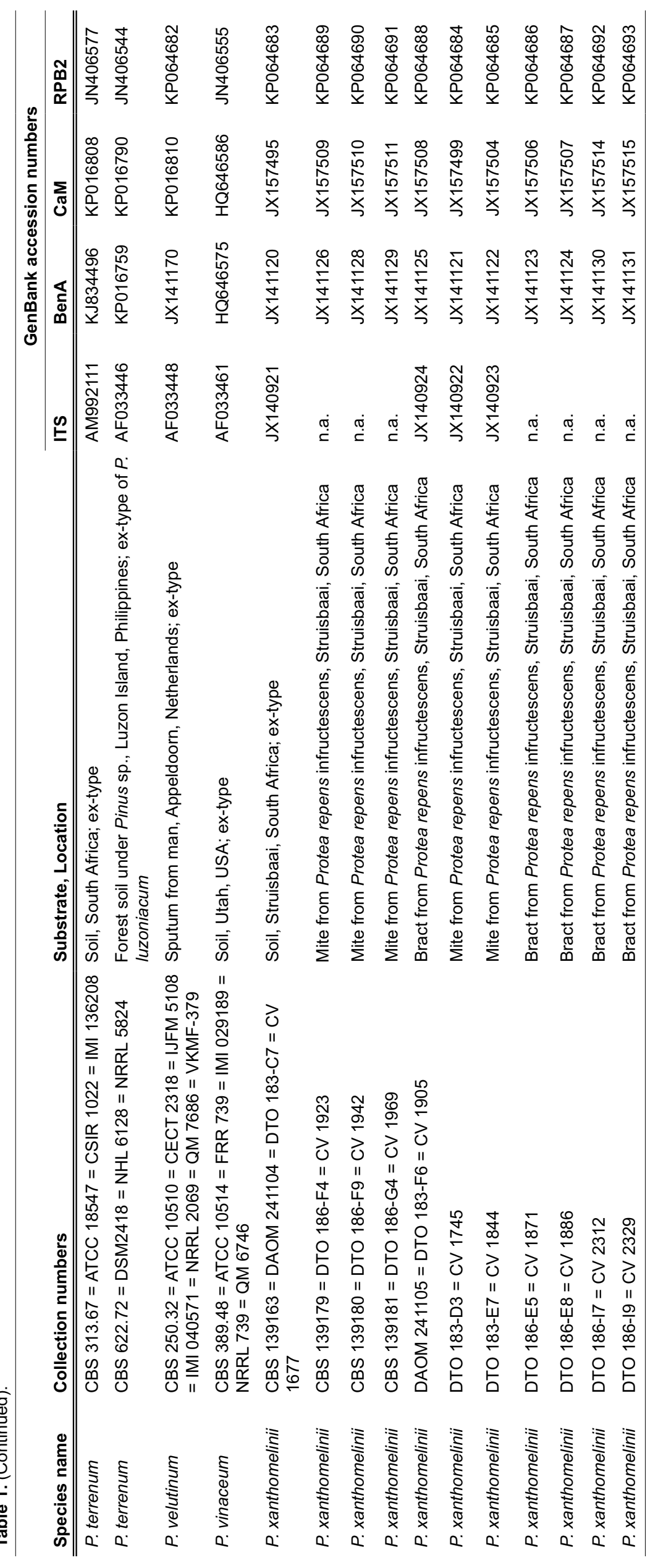


Table 2. Overiview and details used for phylogenetic analyses.

\begin{tabular}{|c|c|c|c|c|c|}
\hline & & \multicolumn{4}{|c|}{ Dataset } \\
\hline & & $P$. citreonigrum clade & P. corylophilum clade & P. melinii clade & P. parvum clade \\
\hline \multirow{3}{*}{ BenA dataset } & Length (bp) & 449 & 420 & 454 & 427 \\
\hline & Substitution model (BI) & $K 80+G$ & $S Y M+G$ & $H K Y+G$ & $S Y M+G$ \\
\hline & Substitution model (ML) & $\mathrm{K} 2+\mathrm{I}$ & $\mathrm{K} 2+\mathrm{G}$ & $\mathrm{K} 2+\mathrm{G}$ & $\mathrm{K} 2+\mathrm{G}$ \\
\hline \multirow{3}{*}{ CaM dataset } & Length (bp) & 495 & 504 & 490 & 530 \\
\hline & Substitution model (BI) & $\mathrm{K} 80+\mathrm{I}$ & $S Y M+I$ & $\mathrm{GTR}+\mathrm{G}$ & $G T R+1+G$ \\
\hline & Substitution model (ML) & $\mathrm{K} 2+\mathrm{G}$ & $\mathrm{K} 2+\mathrm{G}$ & $\mathrm{K} 2+\mathrm{G}$ & $\mathrm{K} 2+\mathrm{G}$ \\
\hline \multirow{3}{*}{$R P B 2$ dataset } & Length (bp) & 877 & 695 & 838 & 888 \\
\hline & Substitution model (BI) & GTR+I+G & $S Y M+1+G$ & $S Y M+G$ & $\mathrm{GTR}+1+\mathrm{G}$ \\
\hline & Substitution model (ML) & $\mathrm{K} 2+\mathrm{G}$ & $\mathrm{K} 2+\mathrm{G}$ & $\mathrm{TN} 93+\mathrm{G}$ & $\mathrm{K} 2+\mathrm{G}$ \\
\hline \multirow{3}{*}{ Concatenated dataset } & Length (bp) & 1821 & 1619 & 1782 & 1845 \\
\hline & Substitution model (BI) & $\mathrm{GTR}+\mathrm{G}$ & $S Y M+I+G$ & $\mathrm{GTR}+1+\mathrm{G}$ & $\mathrm{GTR}+1+\mathrm{G}$ \\
\hline & Substitution model (ML) & $\mathrm{K} 2+\mathrm{I}$ & TN93+G & $\mathrm{K} 2+\mathrm{G}$ & $\mathrm{T} 92+1+\mathrm{G}$ \\
\hline
\end{tabular}

P. atrolazulinum (321 total, six each from air and soil) in Protea repens infructescences and from their mite communities was especially apparent.

\section{Phylogeny}

An ITS phylogeny (Fig. 1) was used to compare all ex-type strains from section Exilicaulis species and sort them into smaller clades for the BenA, CaM, RPB2 and combined phylogenies (Figs 2-6). The aligned ITS barcode data set was 526 bp long with $\mathrm{K} 2+\mathrm{G}+\mathrm{I}$ and $\mathrm{GTR}+\mathrm{G}+\mathrm{I}$ the most suitable substitution models for $\mathrm{ML}$ and $\mathrm{BI}$ respectively. Table 2 summarises the most suitable substitution models used for the remaining phylogenies. The ITS phylogeny distributed strains into six main clades, similar to the results of Houbraken \& Samson (2011). Species with biverticillate conidiophores were resolved in a well-supported clade distinct from monoverticillate species. We also divided the biverticillate clade into subclades, referred to here as the $P$. melinii- and $P$. corylophilum-clades, to present the data concisely. The monoverticillate species resolved in four clades, referred to here as the P. restrictum-, P. citreonigrum-, $P$. decumbens-, and $P$. parvum-clades. ITS performs poorly as a DNA barcode marker in the $P$. melinii-, $P$. corylophilum-, and $P$. restrictum-clades, although it still can be used to identify the important $P$. corylophilum. On the other hand, ITS can successfully identify all species from the $P$. citreonigrum-, $P$. decumbens-, and $P$. parvum-clades.

Penicillium melinii-clade (Fig. 2) - Phylogenetic data divided the fynbos strains into three clades, two representing new species described below as $P$. hemitrachum and $P$. xanthomelinii. The remaining strains were placed in a clade with $P$. melinii and $P$. radulatum. Although there is sequence variation within the clade, phylogenies were in conflict and following GCPSR we identified our strains as $P$. melinii and consider $P$. radulatum a synonym. Ex-type strains of $P$. terrenum, $P$. chalybeum, and $P$. luzoniacum were included in a coherent clade and we consider them to represent one species with $P$. terrenum the oldest name. Similarly, we consider $P$. flavidostipitatum and $P$. turris-painense synonyms of $P$. namyslowskii, while $P$. corynephorum and $P$. sabulosum are synonyms of $P$. smithii.

Penicillium corylophilum-clade (Figs 3-4) - The largest group of fynbos isolates belong to this clade, with nine species identified. GCPSR revealed seven coherent clades distinct from known species, which are introduced as new species below. Penicillium repensicola and $P$. subturcoseum strains were consistently resolved in coherent clades as close relatives. Penicillium momoii and $P$. cravenianum were represented by single isolates that shifted phylogenetic positions depending on the gene used. However, the combined phylogeny places $P$. cravenianum as a close relative to $P$. pagulum, while $P$. momoii seems most similar to $P$. rubefaciens. Sequence variation was observed in the clades described as $P$. atrolazulinum, $P$. consobrinum and $P$. pagulum. However, no coherent clades were observed within strains of $P$. atrolazulinum and $P$. consobrinum. For $P$. pagulum, only two strains were isolated and although they exhibited sequence variation, they were morphologically identical. Remaining fynbos isolates were identified as $P$. corylophilum and $P$. rubefaciens. Ex-type strains of $P$. candidofulvum, $P$. coeruleoviride, $P$. corylophilum, $P$. chloroleucon, $P$. citreovirens, $P$. humuli, and $P$. obscurum clustered together. Again, this clade displays sequence variation, but the only consistent branch is the one encompassing the entire clade. As a result, we accept $P$. corylophilum as the correct name for the clade and consider the remaining species synonyms.

Penicillium citreonigrum-clade (Fig. 5) - Based on the multigene analyses, we accept three species in this clade, namely $P$. citreonigrum, $P$. cinerascens and $P$. citreosulfuratum. Penicillium toxicarium (CBS 351.51) was recently shown to be synonymous with $P$. trzebinskii (Houbraken et al. 2014). On the other hand, Serra et al. (2008) pointed out that $P$. 
AM992111 P. terrenum CBS $313.67^{\top}$

JX140921 P. xanthomelinii CBS $139163^{\top}$

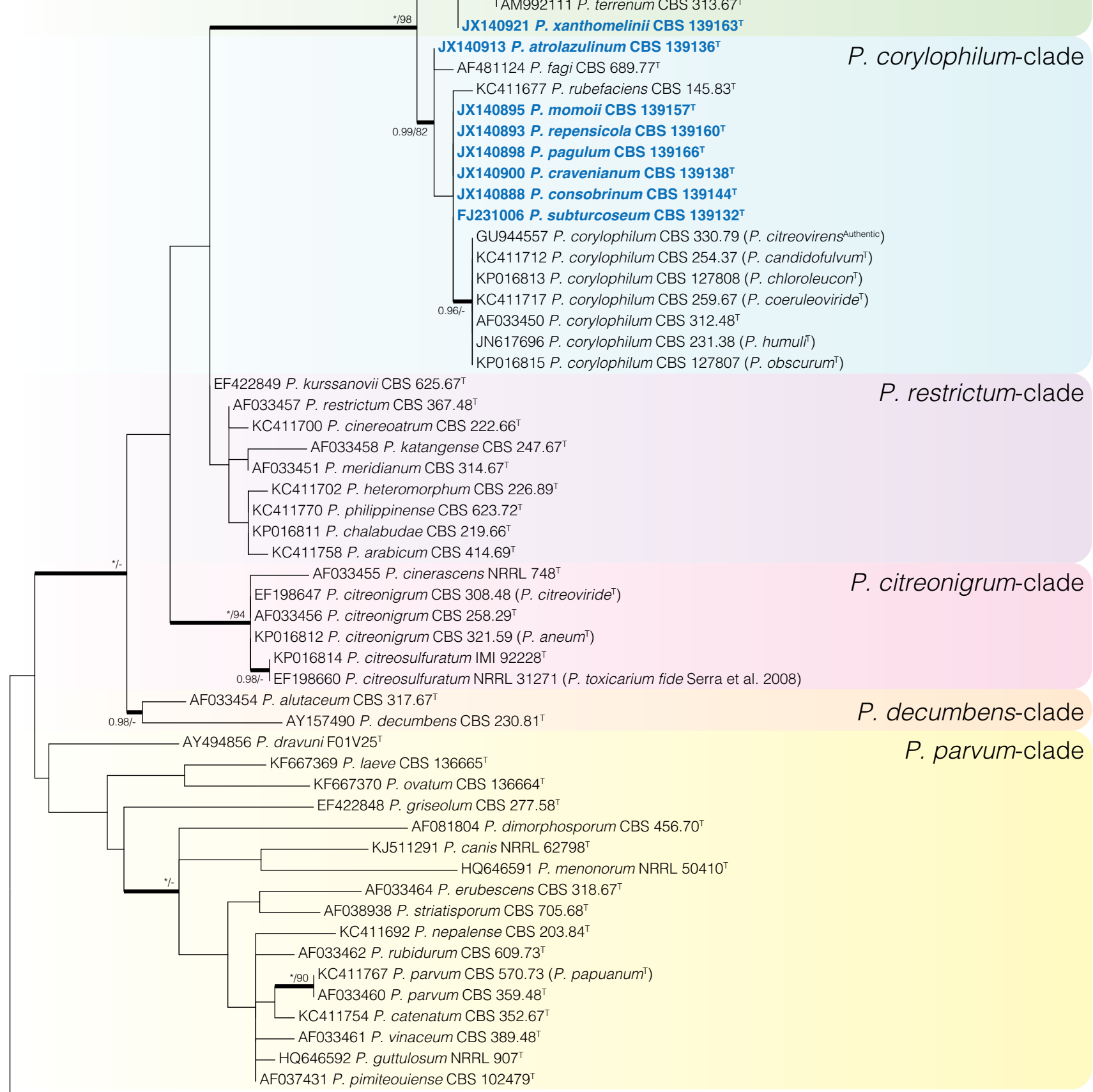

EF422849 P. kurssanovii CBS 625.67

AF033457 P. restrictum CBS 367.48

- KC411702 P heteromorphum CBS 226.89

KC411770 P. philippinense CBS 623.72

KP016811 P. chalabudae CBS 219.66

P. arabicum CBS 414.69

P. 3455 P. cinerascens NRRL 748

EF198647 P. citreonigrum CBS 308.48 (P. citreoviride')

$P$. citreonigrum-clade

KP016812 P. citreonigrum CBS 321.59 ( $P$. aneum ${ }^{\top}$

KP016814 P. citreosulfuratum IMI 92228

198660 P. citreosulfuratum NRRL 31271 ( $P$. toxicarium fide Serra et at. 2008)

\section{P. parvum-clade}

AF037431 P. pimiteouiense CBS 102479

Fig. 1. Phylogenetic tree of Penicillium sect. Exilicaulis species ex-type strains using the ITS region. Penicillium toxicarium (CBS 351.51 ) was chosen as outgroup. Posterior probabilities (pp) and/or bootstrap values (bs) higher than 0.95 and 80 respectively, are given above thickened branches. Names in bold indicate fynbos strains, names in blue indicate new species strains, $T=$ ex-type strain. 

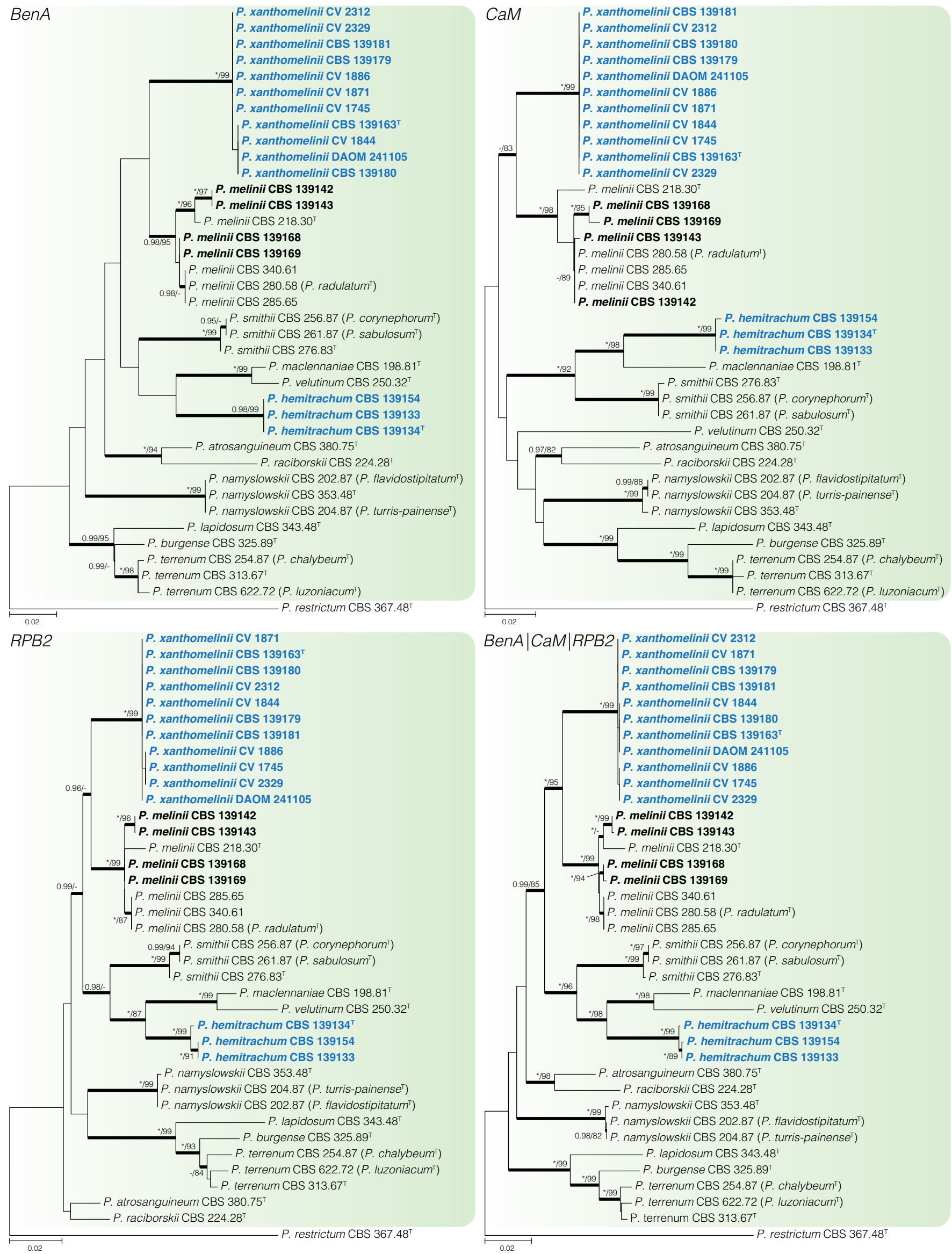

Fig. 2. Phylogenetic tree of Penicillium sect. Exilicaulis species belonging in the P. melinii-clade using BenA, CaM, RPB2 and combined data sets. Penicillium restrictum was chosen as outgroup. Posterior probabilities (pp) and/or bootstrap values (bs) higher than 0.95 and 80 respectively, are given above thickened branches. Names in bold indicate fynbos strains, names in blue indicate new species strains, $T=$ ex-type strain. 

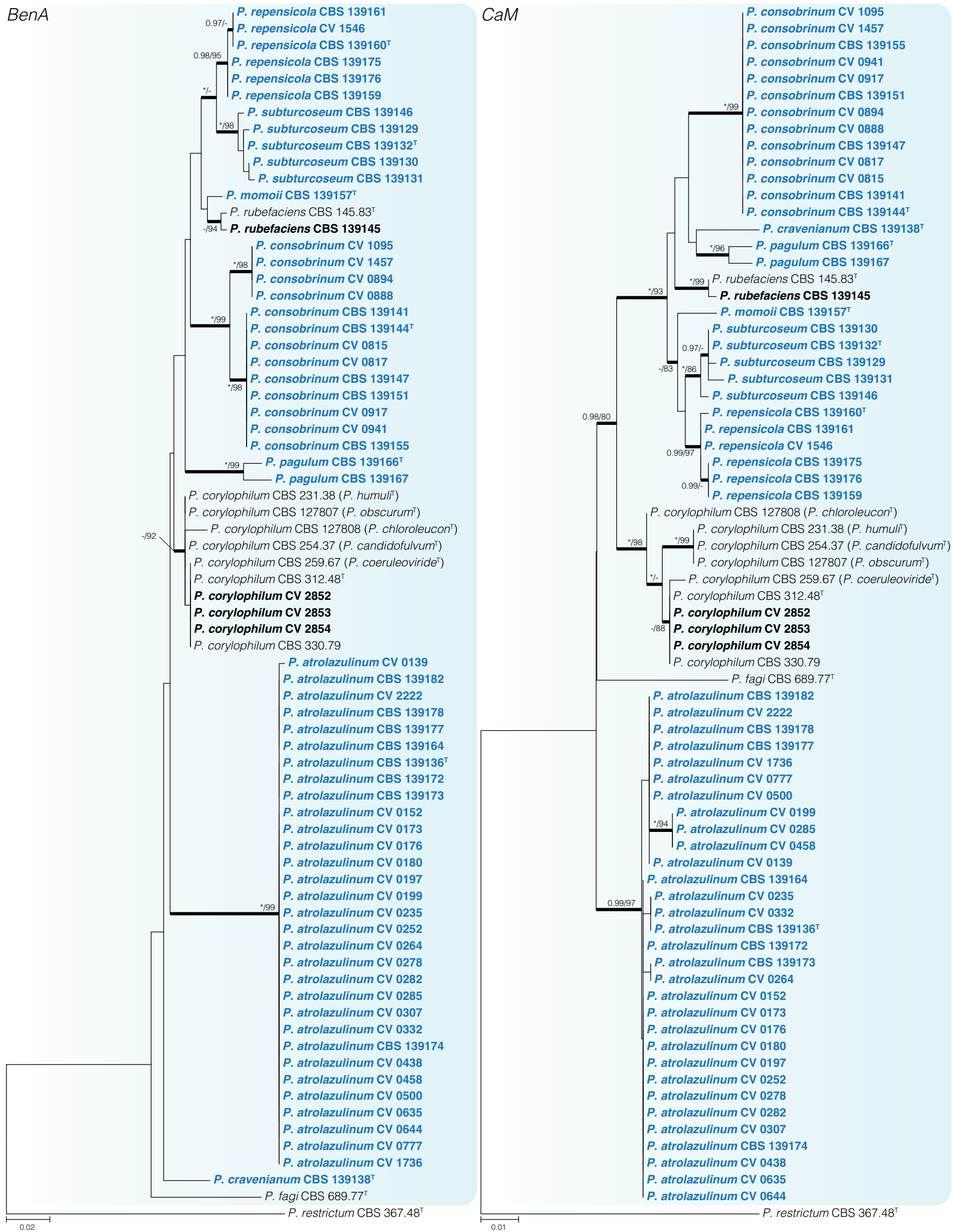

ii CBS 139157

befaciens CBS 13914

P. consobrinum CBS 13914

P. consobrinum CBS $139144^{\top}$

consobrinum CV 0815

P. consobrinum CV 0917

P. consobrinum CV 0941

consobrinum CBS 139155

P. pagulum CBS 139166

P. corylophilum CBS $231.38(P$. humuli $)$

corylophilum CBS 127807 ( $P$. obscurum $)$

corylophilum CBS 254.37 (P. candidofulvum ${ }^{\top}$ )

corylophilum CBS 259.67 ( $P$. coeruleoviride')

P. atrolazulinum CV 0139

P. atrolazulinum CBS 139182

Ptrolazulinum CV 2222

(azulinum CV 0152

P. atrolazulinum CV 0278

atrolazulinum CV 0307

zulinum CV 0332

azulinum CBS 139174

P. atrolazulinum CV 0777

Fig. 3. Phylogenetic tree of Penicillium sect. Exilicaulis species belonging in the $P$. corylophilum-clade using BenA and CaM. Penicillium restrictum was chosen as outgroup. Posterior probabilities (pp) and/or bootstrap values (bs) higher than 0.95 and 80 respectively, are given above thickened branches. Names in bold indicate fynbos strains, names in blue indicate new species strains, $T=$ ex-type strain. 


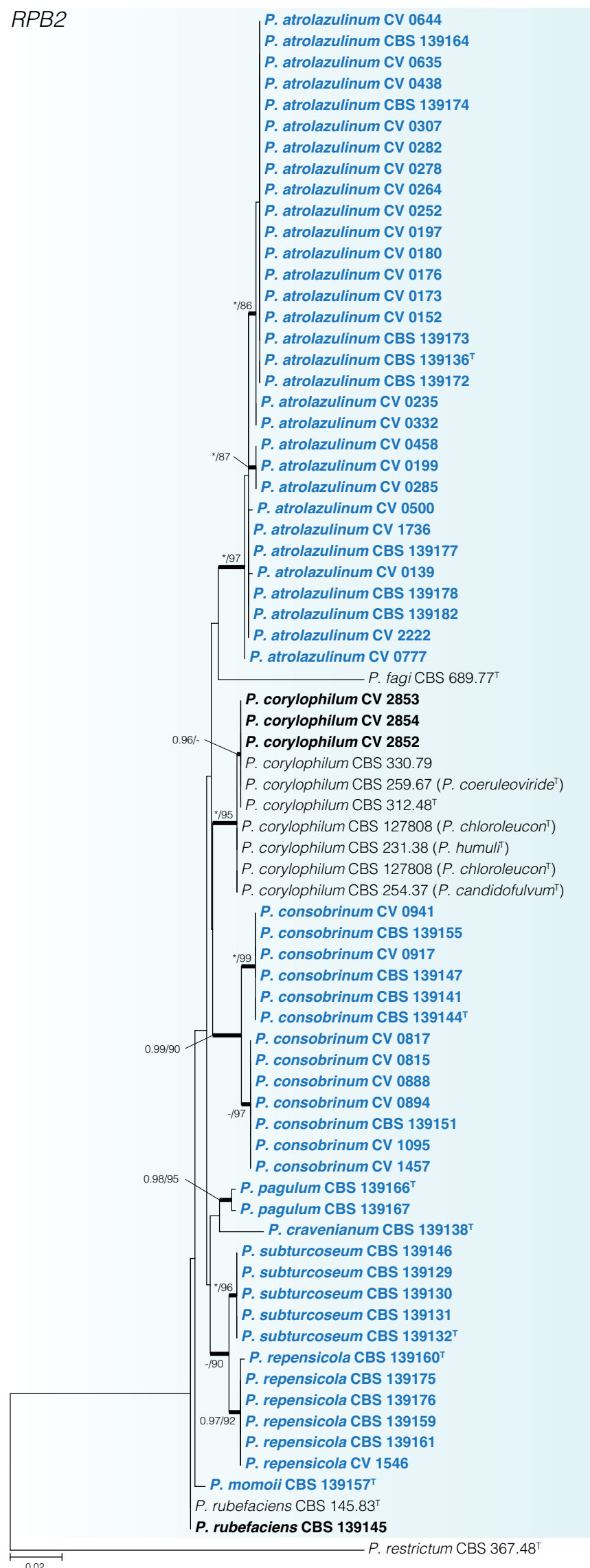

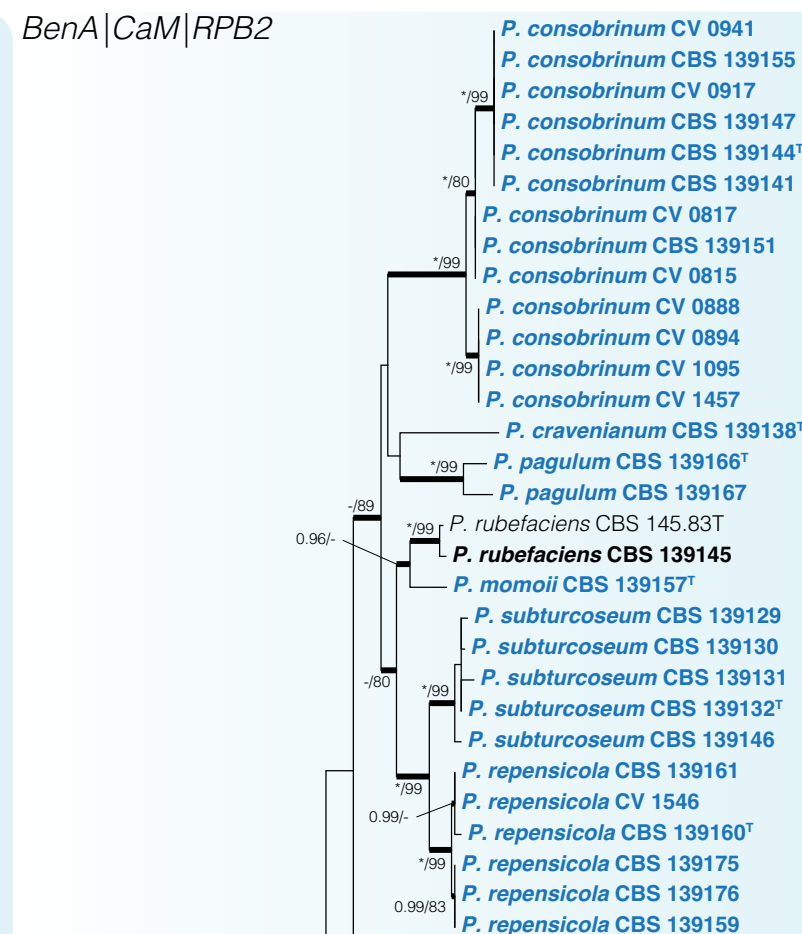

- P. corylophilum CBS 127808 ( $P$. chloroleucon $\left.{ }^{\top}\right)$

P. corylophilum CBS 127808 ( $P$. chloroleucon ${ }^{\top}$ )

P. corylophilum CBS 254.37 (P. candidofulvum ${ }^{\top}$ )

${ }_{198} 1$ P. corylophilum CBS 231.38 ( $P$. humuli $)$

P. corylophilum CBS $312.48^{\top}$

P. corylophilum CV 2852

P. corylophilum CV 2853

*/95 P. corylophilum CV 2854

P. corylophilum CBS 259.67 (P. coeruleoviride ${ }^{\top}$ )

P. corylophilum CBS 330.79

P. atrolazulinum CV 0285

*/98 P. atrolazulinum CV 0458

P. atrolazulinum CV 0199

P. atrolazulinum CV 0777

P. atrolazulinum CV 0139

P. atrolazulinum CV 2222

P. atrolazulinum CBS 139182

P. atrolazulinum CBS 139178

P. atrolazulinum CBS 139177

P. atrolazulinum CV 1736

$P$. atrolazulinum CV 0500

P. atrolazulinum CV 0235

P. atrolazulinum CV 0332

P. atrolazulinum CBS $139136^{\top}$

P. atrolazulinum CBS 139164

P. atrolazulinum CV 0644

P. atrolazulinum CV 0635

P. atrolazulinum CBS 139172

$P$. atrolazulinum CV 0152

P. atrolazulinum CV 0173

P. atrolazulinum CV 0176

P. atrolazulinum CV 0180

P. atrolazulinum CV 0197

P. atrolazulinum CV 0252

P. atrolazulinum CV 0278

$P$. atrolazulinum CV 0282

P. atrolazulinum CV 0307

P. atrolazulinum CBS 139174

P. atrolazulinum CV 0438

P. atrolazulinum CBS 139173

P. atrolazulinum CV 0264 P. fagi CBS $689.77^{\circ}$ P. restrictum CBS 367.48

Fig. 4. Phylogenetic tree of Penicillium sect. Exilicaulis species belonging in the $P$. corylophilum-clade using RPB2 and combined data sets. Penicillium restrictum was chosen as outgroup. Posterior probabilities (pp) and/or bootstrap values (bs) higher than 0.95 and 80 respectively, are given above thickened branches. Names in bold indicate fynbos strains, names in blue indicate new species strains, $T=$ ex-type strain. 

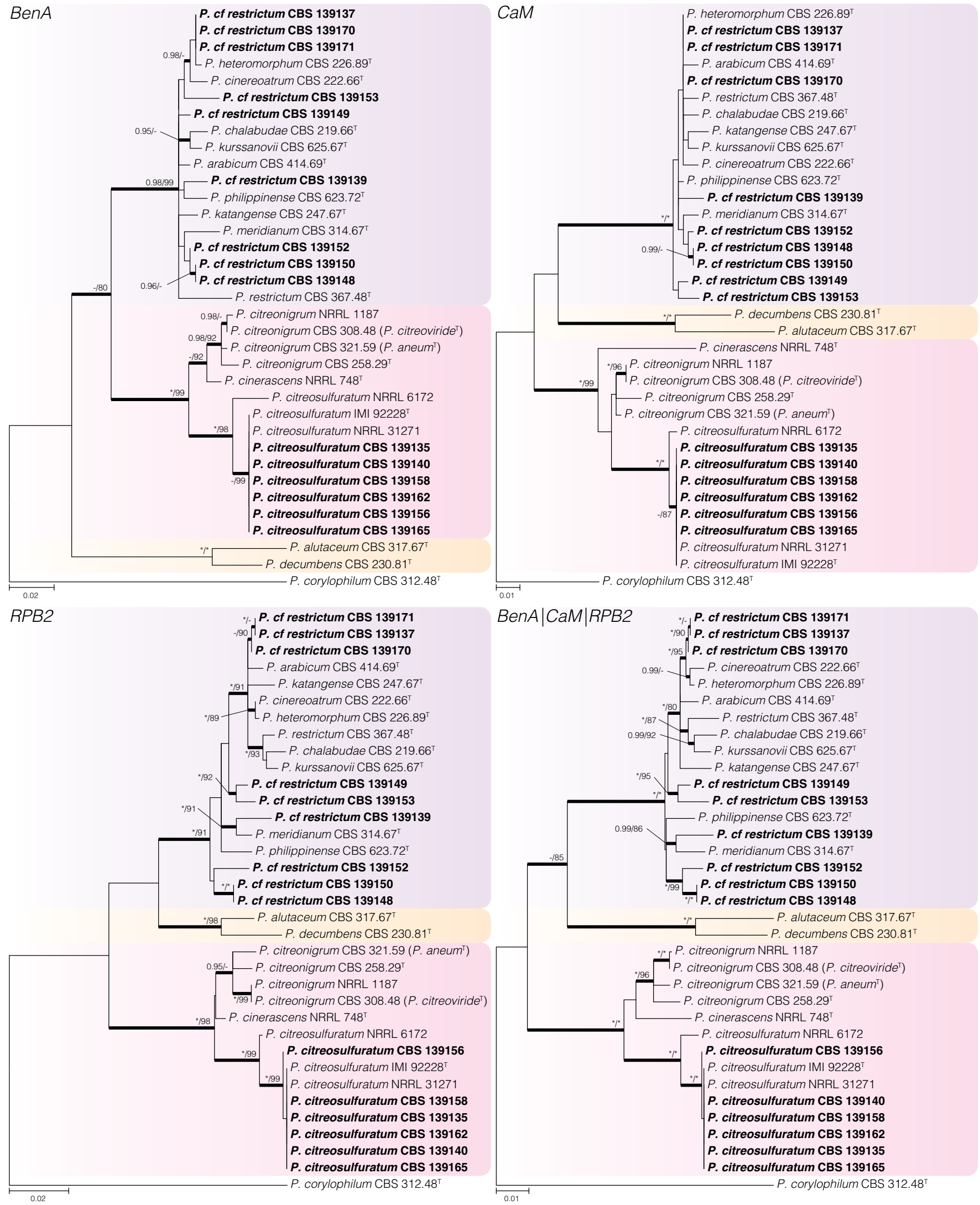

Fig. 5. Phylogenetic tree of Penicillium sect. Exilicaulis species belonging in the $P$. citreonigrum-, $P$. decumbens- and $P$. restrictum-clades using BenA, CaM, RPB2 and combined data sets. Penicillium corylophilum was chosen as outgroup. Posterior probabilities (pp) and/or bootstrap values (bs) higher than 0.95 and 80 respectively, are given above thickened branches. Names in bold indicate fynbos strains, $T=$ ex-type strain. 

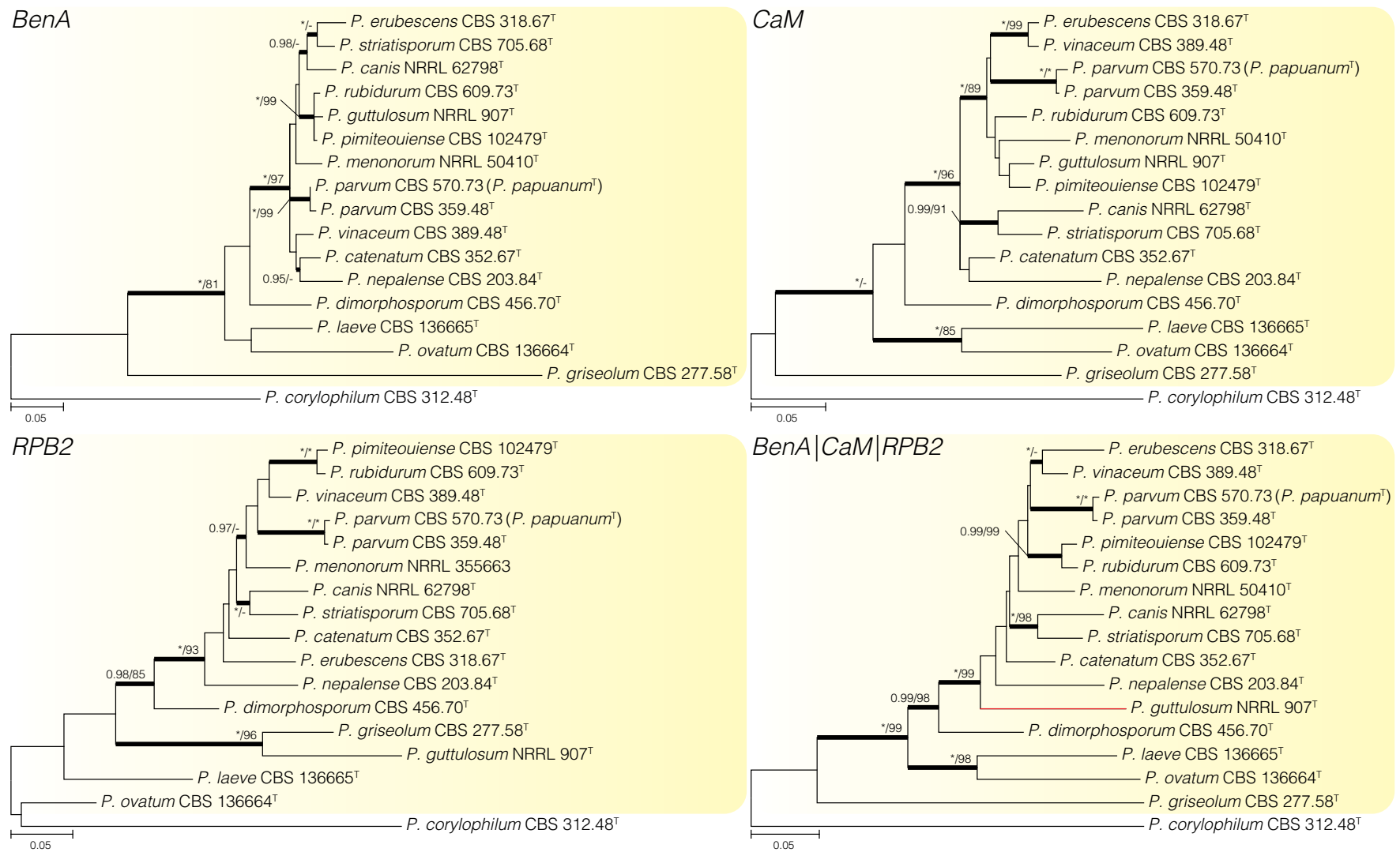

Fig. 6. Phylogenetic tree of Penicillium sect Exilicaulis species belonging in the $P$. parvum-clade using BenA, CaM, RPB2 and combined data sets. Penicillium corylophilum was chosen as outgroup. Posterior probabilities (pp) and/or bootstrap values (bs) higher than 0.95 and 80 respectively, are given above thickened branches. Names in bold indicate fynbos strains, names in blue indicate new species, $T=$ ex-type strain. In the combined phylogeny, the RPB2 sequence (KF900194) for P. menonorum (NRRL 50410) was too short for the phylogeny and was replaced with a representative sequence (KF900193) from different strain (NRRL 35663).

toxicarium should be considered a distinct species and closely related to $P$. citreonigrum; unfortunately, they did not identify which strain in their analysis represented the ex-type. Here we show that strains they named as $P$. toxicarium cluster in a clade with $P$. citreosulfuratum, the latter representing the oldest and thus correct name for the species. Penicillium cinerascens is also shown to be a distinct species and the closest relative of $P$. citreonigrum.

Penicillium decumbens-clade (Fig. 5) - Penicillium decumbens and $P$. alutaceum were resolved as close relatives in a distinct clade and all genes easily distinguish between the two.

Penicillium restrictum-clade (Fig. 5) - This clade includes nine previously described species in a complex and fynbos isolates may represent as many as six new species. However, strains isolated from house dust identified as " $P$. restrictum" (Visagie et al. 2014a) potentially also represent new species and from discussions with colleagues we are aware that there may be even more. Therefore we temporarily identify the fynbos strains as $P$. cf. restrictum, with the suggestion that this complex needs a comprehensive revision. Penicillium arabicum (syn. P. decumbens fide Pitt 1980), P. albocinerascens (syn. P. adametzii fide Pitt 1980), and $P$. kurssanovii (syn. P. restrictum fide Pitt 1980) were not included in the list of accepted species by Visagie et al. (2014b). From our phylogenies, it is clear that they represent distinct species within the species concept currently adopted in this particular clade.

Penicillium parvum-clade (Fig. 6) - None of the fynbos strains resolved in this clade. Our phylogeny confirms the results of Houbraken \& Samson (2011), who considered $P$. papuanum a synonym of $P$. parvum.

\section{Morphology}

Based on morphological characters, Penicillium sect. Exilicaulis can be split into two parts that correlate with the two main groups in the phylogenies. Species from the $P$. corylophilum and $P$. melinii clades typically produce biverticillate conidiophores with metulae on one conidiophore which are often of unequal length. The remaining clades contain species that typically produce non-vesiculate monoverticillate conidiophores with short stipes.

Fynbos isolates of the biverticillate clades typically produce conidiophores with rough stipes (but are smooth in $P$. corylophilum), and metulae and conidia which are roughened. Morphological differences observed were consistently supported by the phylogenies. Strains identified as $P$. melinii produce typical divergent, slender conidiophores with spinose, globose conidia. The rough walled phialides observed in fynbos isolates have not previously been observed in this species. All strains examined in this study produced a brownish colony reverse on CYA and sometimes produced brownish to red exudates on top 
of the colonies. Strains identified as $P$. corylophilum had smooth walled stipes, the only biverticillate species in this section with this character state. The strain identified as $P$. rubefaciens (CBS 139145) was identical with the ex-type strain (CBS 145.83). Penicillium rubefaciens sporulates poorly and grows rather restrictedly on CYA at $25^{\circ} \mathrm{C}$ attaining $15-23 \mathrm{~mm}$ diam, recalling the observations of Quintanilla (1982). The species is morphologically similar to $P$. consobrinum, $P$. repensicola, $P$. momoii, and $P$. subturcoseum, but can be distinguished by the colony growth rates as discussed below.

Monoverticillate fynbos isolates were identified as $P$. citreosulfuratum and $P$. cf. restrictum. Penicillium citreosulfuratum was one of the most commonly isolated species from inside Protea repens infructescences, but was also obtained from soil and air samples. Because of its prevalence, and the confusion surrounding the species concept and name, which was also commonly found in cork from Portugal but at the time identified as $P$. toxicarium (Serra et al. 2008), we provide a description for the species below. As mentioned, phylogenetically strains identified as $P$. cf. restrictum probably represent new species, but the morphological differences are minor and inconsistent between strains, e.g. CBS 139150, CBS 139170 and CBS 139171 which have both smooth and spiny conidia. Therefore, with currently available data there is not enough evidence to introduce new species names amongst this limited set of strains here.

\section{TAXONOMY}

Penicillium atrolazulinum Visagie \& K. Jacobs, sp. nov.

MycoBank MB811001

(Figs 7-8)

Etymology: Latin, atrolazulinum, dark blue. Named in reference to the characteristic dark blue colony reverse on CYA, MEA and YES.

Diagnosis: Differs from close relatives in having dark colony reverses, while conidiophores have relatively long stipes and metulae that often have a green pigmentation.

Type: South Africa: Stellenbosch, isol. ex air sample, Mar. 2009, isol. C. M. Visagie (CBS H-22043 [dried culture] holotype; CBS 139136 = DAOMC $241083=$ DTO 180-H4 = CV 55 - ex-type cultures).

ITS barcode: JX140913. Alternative identification markers:

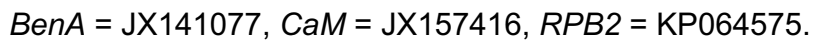

Colony diam, 7 d (in mm): CYA 20-50; CYA $30{ }^{\circ} \mathrm{C}$ 12-27; CYA $37^{\circ} \mathrm{C}$ no growth, rarely up to 5; MEA 30-50; YES 28-47; G25N 6-15; CREA 18-25.

Colony characters: CYA, $25^{\circ} \mathrm{C}, 7 \mathrm{~d}$ : Colonies low to moderately deep, plane to very faintly sulcate; margins low, narrow to wide $(1-5 \mathrm{~mm})$, entire to somewhat irregular; mycelia white, sometimes green when embedded; texture mostly velutinous, floccose regions present; sporulation moderately dense, conidia en masse dull to greyish green (24D4-6; 25D4-6; 25E4-26E4); exudate clear to almost a hazy yellow, sometimes absent; soluble pigment mostly absent, sometimes yellowish orange; reverse dark blue to dark turquoise (23F8-24F8), pale yellow (1A3-2A3) at margin, some strains dark green (27F6-7) at centre, pastel yellow (3A4) at margin. MEA, 25 ${ }^{\circ} \mathrm{C}, 7 \mathrm{~d}$ : Colonies low, plane; margins low to almost subsurface, wide $(4-5 \mathrm{~mm})$, entire; mycelia white, sometimes green when embedded; texture velutinous, floccose areas present; sporulation dense to moderately dense, conidia en masse dull green to greyish green (26E4-6) at centre, greyish green (25D6) near margin, some strains greyish turquoise (24C6E4-5); exudate absent; soluble pigment absent; reverse dark blue to dark turquoise (24F8-25F8), greyish green (29C3) near margin, some strains dark green (28F6-7) at centre, (30C5) near margin. YES, $25{ }^{\circ} \mathrm{C}, 7 \mathrm{~d}$ : Colonies low, to moderately deep, random furrows and ridges present; margins low, wide, regular, entire; mycelia white, sometimes green when embedded; texture velutinous, with loosely funiculose mycelia present; sporulation moderately dense, conidia en masse similar to CYA; exudate absent; soluble pigment absent, some strains yellowish orange; reverse dark blue to dark turquoise (24F8-25F8), pale yellow (1A3-2A3) at margin, some strains dark green $(25 \mathrm{~F} 8)$ at centre, greyish yellow to yellow (3B53B8) near margin. CREA, $25^{\circ} \mathrm{C}, 7 \mathrm{~d}$ : Acid not produced.

Micromorphology: Conidiophores typically borne from green mycelia embedded in medium, stipes mostly hyaline but sometimes green, mostly biverticillate, infrequently terverticillate, monoverticillate side branches sometimes present; Stipes very short to very long, typically rough, a minor proportion smooth to finely rough, $40-400 \times 2.5-4 \mu \mathrm{m}$; Branches two when present, $13-39.5 \times 2.5-4 \mu \mathrm{m}$; Metulae mostly 3-5 per stipe, sometimes only two, divergent 38-69 ${ }^{\circ}$ (52.8 \pm 9.2$), 11-23 \times 2.5-4 \mu \mathrm{m}(15.4 \pm 2.7 \times 3.1 \pm 0.4)$, vesicle 3.5-6 $\mu \mathrm{m}(4.7 \pm 0.5)$; Phialides ampulliform, 6-9 per metula, sometimes up to sixteen, $7-9.5 \times 2.5-4 \mu \mathrm{m}(8.3 \pm 0.6 \times$ $3 \pm 0.3$ ); Conidia smooth, globose to subglobose, $2-3 \times 2-2.5$ $\mu \mathrm{m}(2.3 \pm 0.2 \times 2.2 \pm 0.1)$, average width/length $=0.95, n=32$.

Notes: Penicillium atrolazulinum typically produces conidiophores with roughened stipes borne from greenish to blue submerged mycelia. This green pigmentation is present in some conidiophores, although most are hyaline, and gives this species its most striking feature, the dark turquoise to green colony reverse observed on most media. Although these characters are consistent, infraspecific variation is observed among strains, especially in colony diameter and appearance on CYA. Some strains, for instance, produce yellowish orange soluble pigments, which mask the characteristic reverse colour. Although a large degree of morphological variation is observed, the phylogenies confirm this as one species (Figs 3-4). Morphologically, P. fagi closely resembles the new species, producing dark colony reverses and similar conidiophores (Martinez \& Ramírez 1978). In general, $P$. atrolazulinum produces longer stipes and metulae than $P$. fagi. Also, the green conidiophore stipes sometimes observed in the new species are unreported for $P$. fagi. Phylogenetically, $P$. atrolazulinum is distinct from all previously described species (Figs 3-4). 

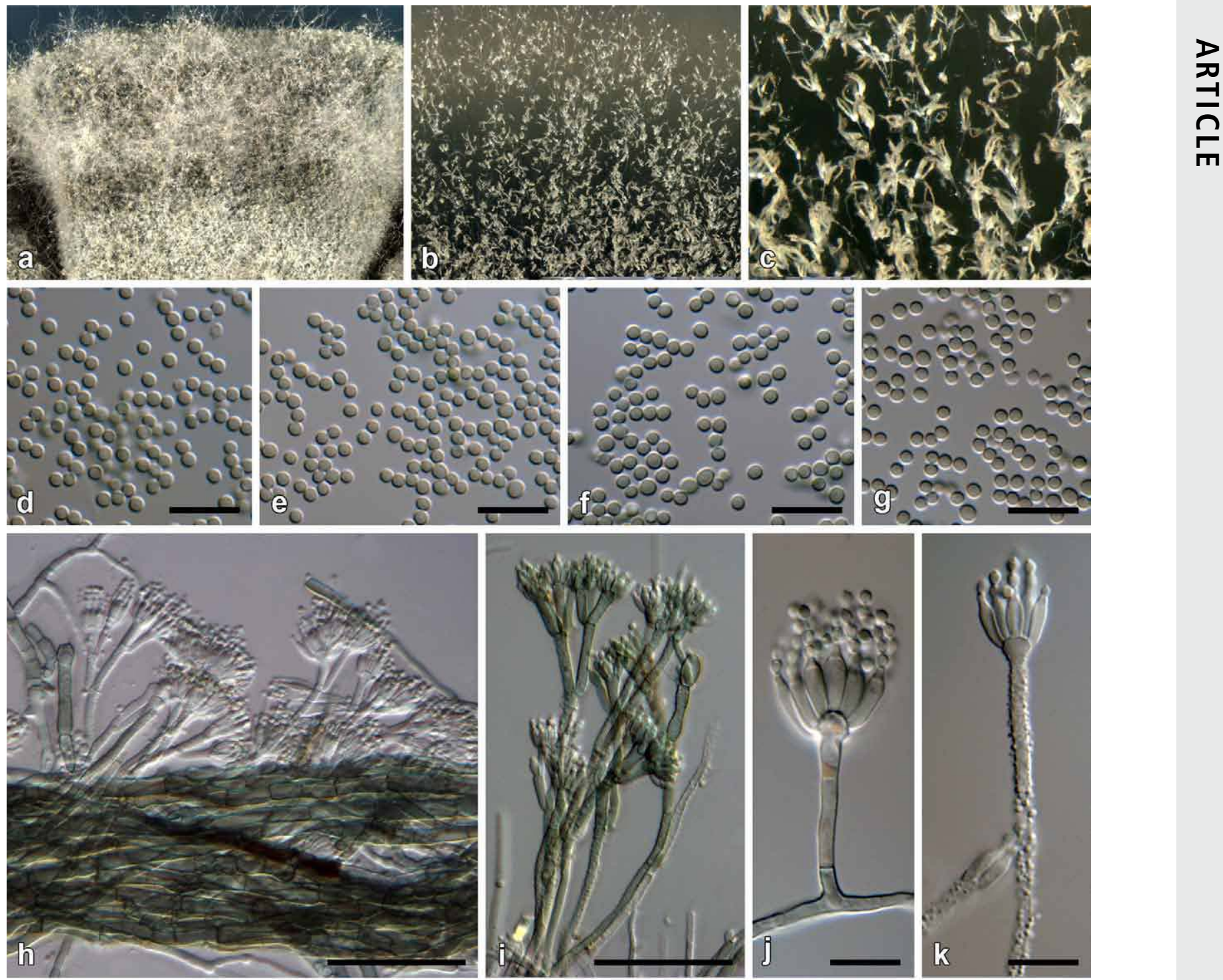

8204

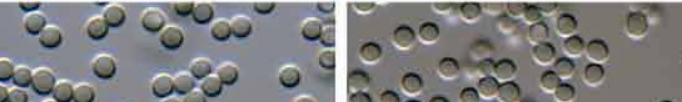

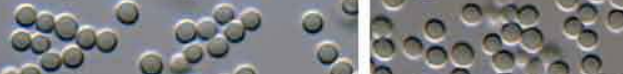

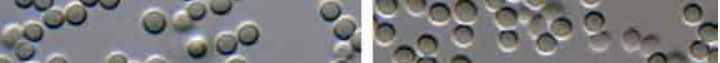
0.00 कo of

205 3900000

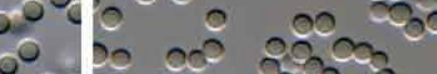

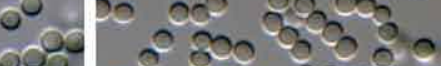

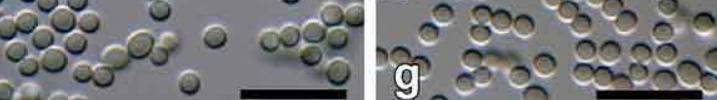
1500000
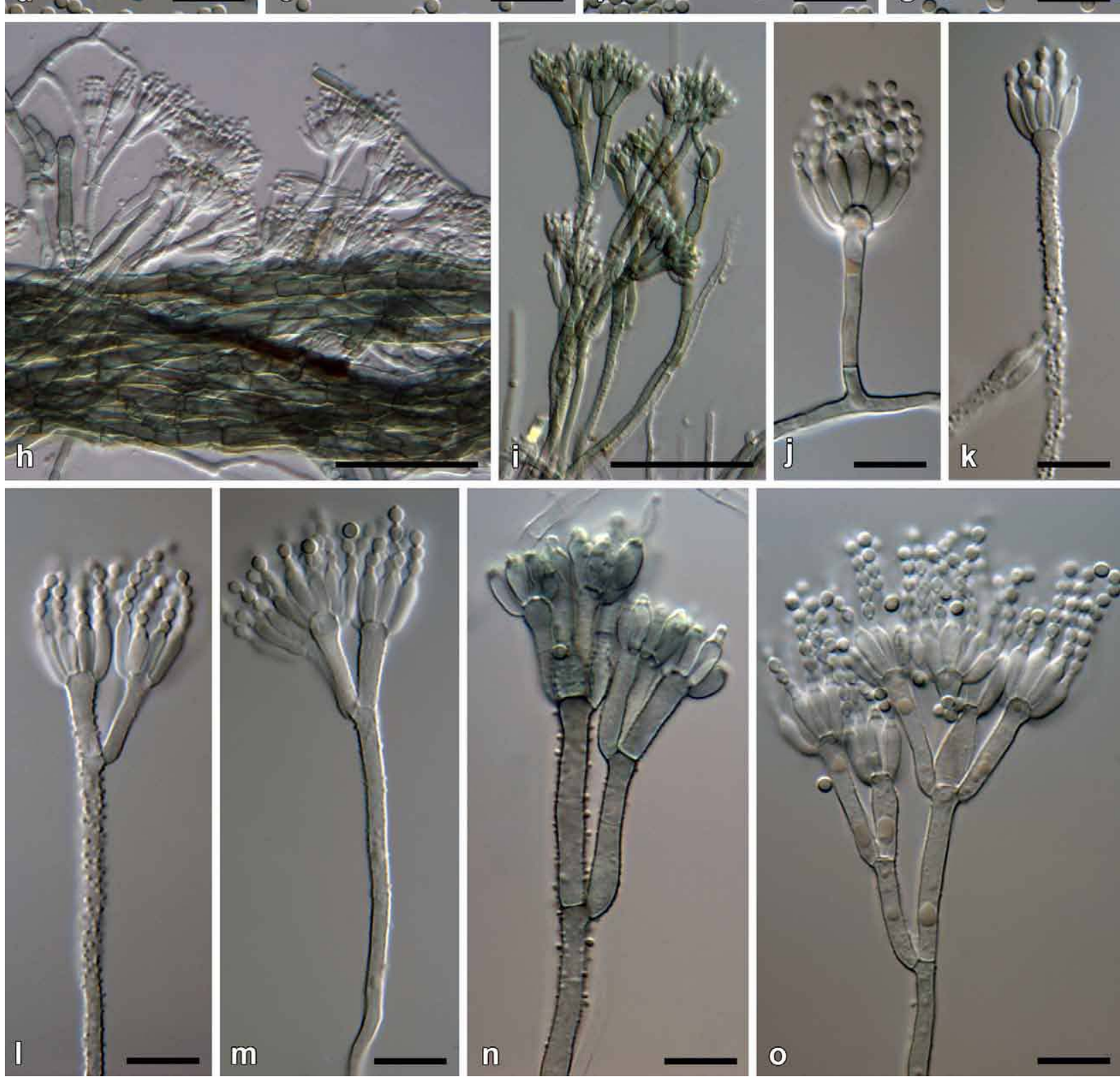

Fig. 8. Penicillium atrolazulinum conidiophores. a. Texture on CYA. b, c. Texture on MEA. d-g. Conidia (d. CBS 139136. e. CV 120. f. CBS 139164. g. CV 1776). h. Green mycelia embedded in medium, from where conidiophores are borne. $\mathrm{i}-\mathrm{o}$. Conidiophores. Bars $\mathrm{h}-\mathrm{i}=50 \mathrm{\mu m} ; \mathrm{d}-\mathrm{g}$, $1-0=10 \mu \mathrm{m}$. 


\section{Penicillium burgense Quintan. ex Visagie, sp. nov. MycoBank MB816641}

Synonym: Penicillium burgense Quintan., Av. Aliment Majora Anim. 30: 176 (1990); nom. inval. (Art. 40.7).

Diagnosis: Differs from its close relatives, $P$. lapidosum and $P$. terrenum, by lacking ascospore production and typically more complexly branched conidiophores. Also, faster growth reported at $37^{\circ} \mathrm{C}$ (Quintanilla 1990).

Type: Spain: highlands north of Burgos, isol. ex soil, date unknown, isol. J.A. Quintanilla (CBS H-22567 [dried culture] - holotype; CBS 325.89 - ex-type cultures).

ITS barcode: KC411736. Alternative identification markers: BenA $=\mathrm{KJ} 834437, \mathrm{CaM}=\mathrm{KP016772}, \mathrm{RPB2}=\mathrm{JN} 406572$.

Notes: Penicillium burgense was invalidly described by Quintanilla (1990) because no collection or herbarium was specified with his "1538" type designation (Art. 40.7). His original strain was sent to the CBS collection (CBS 325.89), the Netherlands. A dried specimen of his strain was used here to correct the typification and application of the name.

\section{Penicillium chalabudae Visagie, nom. nov. MycoBank MB816642}

Replaced name: Penicillium albocinerascens Chalab., Not. Syst. Crypt. Inst. bot. Acad. Sci. USSR 6: 166 (1950); nom. illegit. (Art. 53.1).

Non Penicillium albocinerascens (Maublanc) Biourge, Cellule 33: 100 (1923) fide (Pitt 1980).

Diagnosis: Morphologically this species has all the characters associated with the $P$. restrictum species complex. Multigene analysis confirms the species as unique.

Etymology: Latin, chalabudae, named after T.V. Chalabuda.

Type: Ukraine: Kiev, ex soil sample, isol. T.V. Chalabuda (CBS H-15439 [dried culture] - holotype; CBS $219.66=$ ATCC $18322=$ ATCC $18329=$ FRR $3393=$ VKM F-1037 ex-type cultures).

ITS barcode: KP016811. Alternative identification markers: $B e n A=\mathrm{KP} 016748, \mathrm{CaM}=\mathrm{KP} 016767, \mathrm{RPB2}=\mathrm{KP} 064572$.

Notes: Chalabuda (1950) used the epithet "albocinerascens" for his new species, not realising that Biourge (1923) already used the name. As such, Chalabuda's species is illegitimate (Art. 53.1) and we introduce the new name $P$. chalabudae for $\mathrm{CBS} \mathrm{H}-15439$. The species phylogenetically belongs in the $P$. restrictum species complex and has unique sequences.

Penicillium citreosulfuratum Biourge, Cellule 33: 285 (1923).

MycoBank MB260947

(Fig. 9)

Synonym: Penicillium toxicarium I. Miyake, Rep. Res. Inst. Rice Improvement: 1 (1940); nom. inval. (Art. 39.1).

Non Penicillium toxicarium I. Miyake ex C. Ramírez, Man.
Atlas Penicillia: 125 (1982) = Penicillium trzebinskii K.M. Zalessky 1927 fide (Houbraken et al. 2014).

Type: France: source unknown, Biourge, Cellule 33: fig. 86 n 21 (- lectotype designated here, MBT203135; K(M) IMI 92228 [dried culture] - epitype designated here, MBT203136; IMI 92228 = DTO 290-I4 = Biourge 21 = MUCL 29785 - ex-type cultures).

ITS barcode: KP016814. Alternative identification markers: BenA = KP016753, CaM = KP016777, RPB2 = KP064615.

Colony diam, $7 d$ (in $\mathrm{mm}$ ): CYA 20-25; CYA $30{ }^{\circ} \mathrm{C}$ 20-25; CYA $37{ }^{\circ} \mathrm{C}$ 3-7; MEA 15-18; YES 27-33; G25N 12-15; CREA 10-13.

Colony characters: CYA, $25{ }^{\circ} \mathrm{C}, 7 \mathrm{~d}$ : Colonies radially and concentrically sulcate, moderately deep; margins low, narrow, entire; mycelia yellow; texture floccose; sporulation sparse to moderate, conidia en masse greyish to dark green (25D6F6-26E6); exudate clear, absent in some strains; soluble pigment yellow; reverse yellow (3A6-3A8) to orange-yellow (4B8). MEA, $25^{\circ} \mathrm{C}, 7 \mathrm{~d}$ : Colonies low, plane; margins low, narrow, entire; mycelia white at margins, yellow elsewhere; texture floccose; sporulation moderately dense, conidia en masse dull to greyish green (25E4-26E5); exudate absent; soluble pigment yellow; reverse greenish yellow (1A6) at point of inoculation, fading into greyish yellow (1B6-2B6). YES, $25{ }^{\circ} \mathrm{C}, 7 \mathrm{~d}$ : Colonies low to moderately deep, radially and concentrically sulcate; margins low, very narrow, entire; mycelia white at edges, yellow centrally; texture floccose; sporulation sparse, conidia en masse greenish white (27A2), dull green (27E4) in more dense areas; exudate absent; soluble pigment absent; reverse light yellow to yellow (3A46) to deep yellow (4A8) in some isolates. CREA, $25^{\circ} \mathrm{C}, 7 \mathrm{~d}$ : Acid not produced.

Micromorphology: Conidiophores mostly monoverticillate but sometimes with very short stipes that could be interpreted as subterminal branches, with biverticillate conidiophores sometimes present; Stipes/branches smooth, when terminal $70-290 \times 2-2.5 \mu \mathrm{m}$, when borne sub-terminally $18-90 \times$ 2-2.5 $\mu \mathrm{m}$; Metulae typically 2 , sometimes 3, divergent, 8-29 $\times 1.5-2.5 \mu \mathrm{m}(18 \pm 4.5 \times 2.1 \pm 0.2)$, vesicle $2.5-4 \mu \mathrm{m}(3.4 \pm 0.3)$; Phialides ampulliform, 5-10 per metula, 5-9 × 2-3 $\mu \mathrm{m}$ $(6.7 \pm 0.9 \times 2.4 \pm 0.2)$; Conidia smooth, globose, $1.5-2 \times 1.5-2$ $\mu \mathrm{m}(1.8 \pm 0.1 \times 1.8 \pm 0.1)$, average width/length $=0.98, n=50$.

Notes: Penicillium citreosulfuratum is distinguished by the compact yellow colonies and the soluble pigments produced on most media. The conidiophores are typically smooth walled, short, and slender and sometimes can be interpreted as either monoverticillate with very short stipes or as irregularly biverticillate (divaricate). In the strains examined, most conidiophores were strictly monoverticillate. Penicillium citreosulfuratum is closely related to $P$. citreonigrum and $P$. cinerascens (Figs 1, 5). These three species are morphologically difficult to distinguish. Ex-type strains of $P$. citreonigrum and $P$. cinerascens do not grow at $37{ }^{\circ} \mathrm{C}$, whereas all $P$. citreosulfuratum strains studied had 

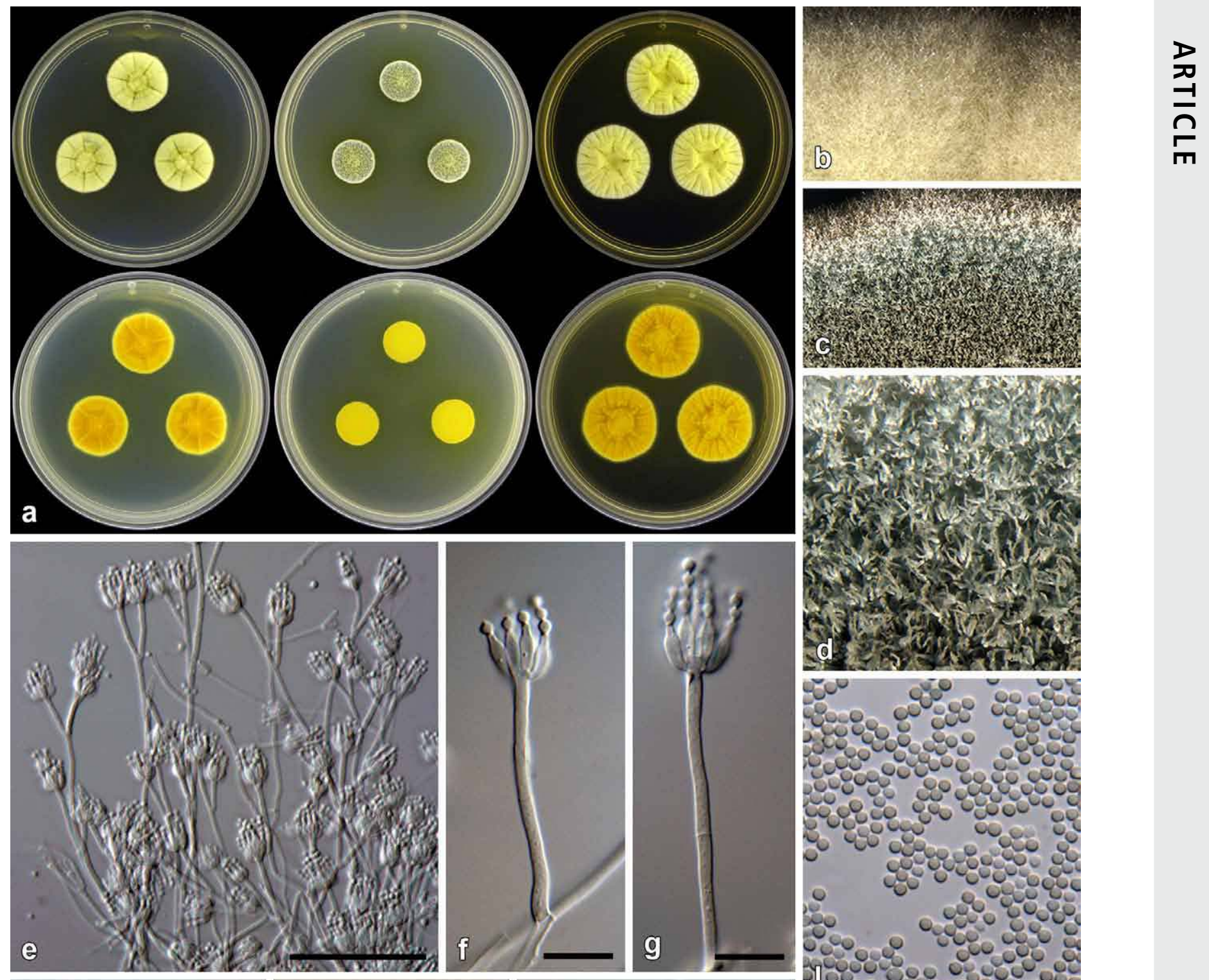

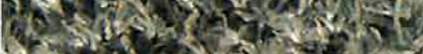

4.

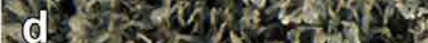

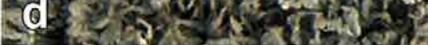

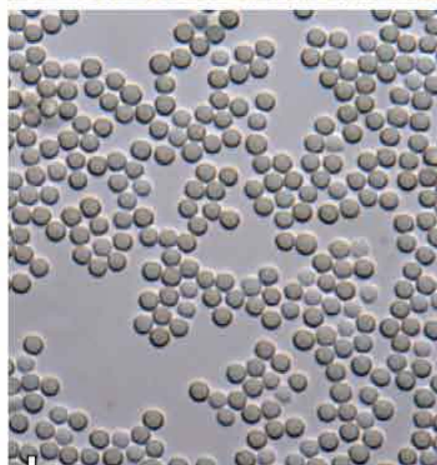
- cos yeojus es

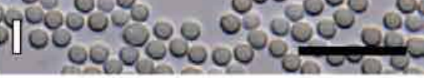
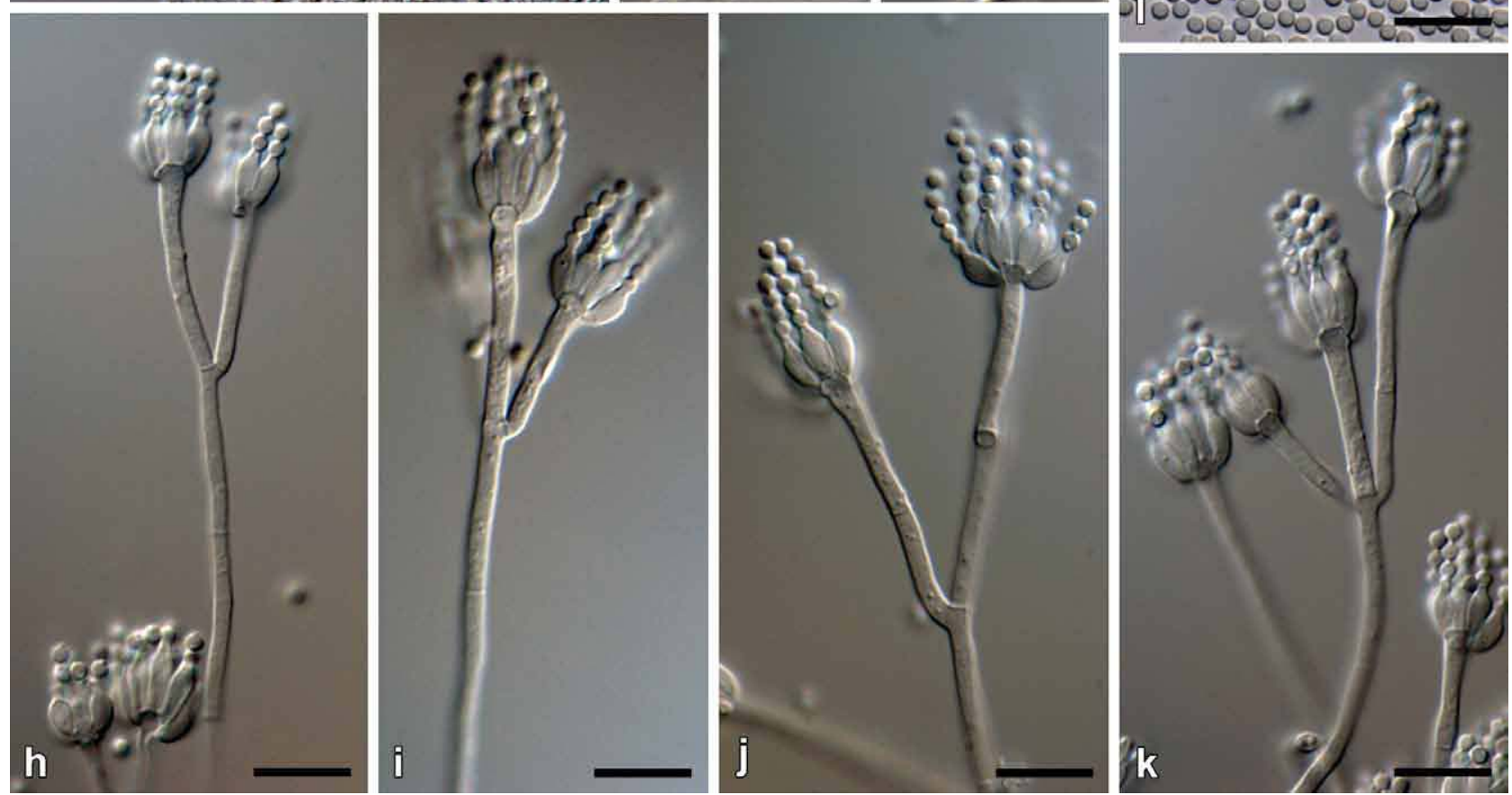

Fig. 9. Penicillium citreosulfuratum (CBS 139135). a. Colonies (top row, left to right: CYA, MEA, YES; bottom row, left to right: CYA reverse, MEA reverse, YES reverse). b. Texture on CYA. c-d. Texture on MEA. e-k. Conidiophores. I. Conidia. Bars e $=50 \mu \mathrm{m}, \mathrm{f}-\mathrm{I}=10 \mu \mathrm{m}$. 
at least restricted growth at that temperature. For accurate identification of these species, we suggest the use of ITS or BenA sequences. Penicillium toxicarium became the informal name for this species following the publication of Serra et al. (2008), but they did not provide information on the type material of that species. $P$. citreosulfuratum is, however, clearly the correct name for this species because it is the oldest name and type material is available.

\section{Penicillium consobrinum Visagie \& K. Jacobs, sp. nov.}

MycoBank MB811002

(Fig. 10)

Etymology: Latin, consobrinum, cousin, named in reference to its close relationship with other species in the $P$. corylophilum clade.

Diagnosis: Differs from close relatives by showing no growth on CYA at $37^{\circ} \mathrm{C}$, but colonies on MEA 48-52 mm.

Type: South Africa: Stellenbosch, isol. ex soil sample, Mar. 2009, isol. C. M. Visagie (CBS H-22045 [dried culture] holotype; CBS 139144 = DAOMC $241072=$ DTO 181-H9 = CV547 - ex-type cultures).

ITS barcode: JX140888. Alternative identification markers: BenA $=\mathrm{JX} 141135, \mathrm{CaM}=\mathrm{JX} 157453, \mathrm{RPB2}=\mathrm{KP} 064619$.

Colony diam, 7 d (in mm): CYA 29-34; CYA $30{ }^{\circ} \mathrm{C} 25-32$; CYA $37^{\circ} \mathrm{C}$ no growth; MEA 48-52; YES 40-45; G25N 11-15; CREA 17-20.

Colony characters: CYA, $25{ }^{\circ} \mathrm{C}, 7 \mathrm{~d}$ : Colonies low to moderately deep, faintly radially sulcate, often having sterile areas at centre, giving colony a greyish green colour; margins low, very narrow (1 mm), entire; mycelia white; texture mostly velutinous, floccose at colony centre; sporulation moderately dense, conidia en masse greyish green (25E6-7) and greyish turquoise (24B3-4); exudate mostly absent, sometimes clear exudate present; soluble pigment absent; reverse brown (5F7-6F7) at centre, becoming pale yellow (4A3) near margin. MEA, $25{ }^{\circ} \mathrm{C}, 7 \mathrm{~d}$ : Colonies low, plane; margins low to subsurface, wide (4 mm), entire; mycelia white; texture velutinous; sporulation dense, conidia en masse greyish green (25E6-7); exudate absent; soluble pigment absent; reverse greyish yellow (2B5) at centre, fading into greyish green to greyish yellow (30B3-C3-4). YES, $25^{\circ} \mathrm{C}, 7 \mathrm{~d}$ : Colonies low to moderately deep, radially and faintly concentrically sulcate, random furrows present; margins low, narrow, entire; mycelia white; texture mostly velutinous, floccose areas present; sporulation moderately dense to dense in regions, conidia en masse similar to CYA; exudate absent; soluble pigment absent; reverse dark green (25F8) at centre, fading into greyish green (28C4) to greyish yellow (1B4) near margin. CREA, $25^{\circ} \mathrm{C}, 7 \mathrm{~d}$ : Acid not produced.

Micromorphology: Conidiophores biverticillate with a minor proportion terverticillate and having subterminal branches; Stipes rough, $75-400 \times 2.5-3.5 \mu \mathrm{m}$; Branches 2 when present, $16-70 \times 2.5-3.5 \mu \mathrm{m}$; Metulae 2-5 per stipe, divergent, sometimes slightly appressed, $25-76^{\circ}(46 \pm 10.4)$, $12-25 \times 2.5-3.5 \mu \mathrm{m}(17 \pm 2.3 \times 2.9 \pm 0.3)$, vesicle $3.5-5.5 \mu \mathrm{m}$ (4.5 \pm 0.5 ); Phialides ampulliform, 12-16 per metula, 6.5-10 $\times$ 2-3.5 $\mu \mathrm{m}(8.3 \pm 0.6 \times 2.8 \pm 0.3)$; Conidia finely roughened, globose, $2-3 \times 2-3 \mu \mathrm{m}(2.3 \pm 0.2 \times 2.3 \pm 0.2)$, average width/ length $=0.97, n=79$.

Notes: Penicillium consobrinum typically produces colonies with a dark green reverse on YES and a brown reverse on CYA. Conidiophores are rough walled with finely roughened, globose conidia. Colony characters closely resemble those of $P$. corylophilum, but the latter produces smooth walled conidiophores. Phylogenetically, $P$. consobrinum belongs to a clade with $P$. cravenianum, $P$. pagulum, $P$. repensicola, $P$. rubefaciens, $P$. momoii, and $P$. subturcoseum. In that clade, $P$. cravenianum is distinguished from other species by the slow growth on MEA (16-20 mm). Penicillium pagulum, on the other hand, grows poorly on CYA at $25^{\circ} \mathrm{C}(15-20 \mathrm{~mm})$. Penicillium rubefaciens does not grow on CYA at $37^{\circ} \mathrm{C}$, whereas $P$. pagulum has restricted growth $(1-5 \mathrm{~mm})$ at that temperature. Penicillium consobrinum also does not grow at $37^{\circ} \mathrm{C}$, but on MEA grows faster than $P$. rubefaciens (48-52 vs. 25-30 mm). The remaining three species, $P$. repensicola $(7-15 \mathrm{~mm}), P$. momoii $(5-8 \mathrm{~mm})$, and $P$. subturcoseum (1-2 mm), can grow on CYA at $37^{\circ} \mathrm{C}$, with $P$. repensicola strains consistently growing fastest, while $P$ momoii grew faster (only based on the one strain) than $P$. subturcoseum. The sporulation of $P$. repensicola strains is also consistently much denser on CYA at $25{ }^{\circ} \mathrm{C}$ than other species in this clade. Penicillium momoii grows only slightly more restrictedly on MEA and G25N compared to $P$. subturcoseum. Morphological differences are sometimes minor, which makes identification difficult, but these differences correlate with the phylogenies applying GCPSR (Figs 3-4).

Penicillium cravenianum Visagie \& K. Jacobs, sp. nov. MycoBank MB811003

(Fig. 11)

Etymology: Latin, cravenianum, named after Danie Craven, former president of the South African Rugby Board and exstudent of Stellenbosch University. The species was isolated from the foot of the mountain next to the Danie Craven Rugby Stadium.

Diagnosis: Differs from close relatives by showing restricted growth on MEA at $25^{\circ} \mathrm{C}(16-20 \mathrm{~mm})$.

Type: South Africa: Stellenbosch, isol. ex soil sample, Mar. 2009, isol. C. M. Visagie (CBS H-22044 [dried culture] holotype; CBS 139138 = DAOMC 241082 = DTO 180-15 = CV 92 - ex-type cultures).

ITS barcode: JX140900. Alternative identification markers: BenA $=$ JX141076, CaM = JX157418, RPB2 = KP064636.

Colony diam, 7 d (in mm): CYA 28-30; CYA $30{ }^{\circ} \mathrm{C} 25-28$; CYA $37^{\circ} \mathrm{C}$ no growth; MEA 16-20; YES 28-34; G25N 8-11; CREA 19-22. 

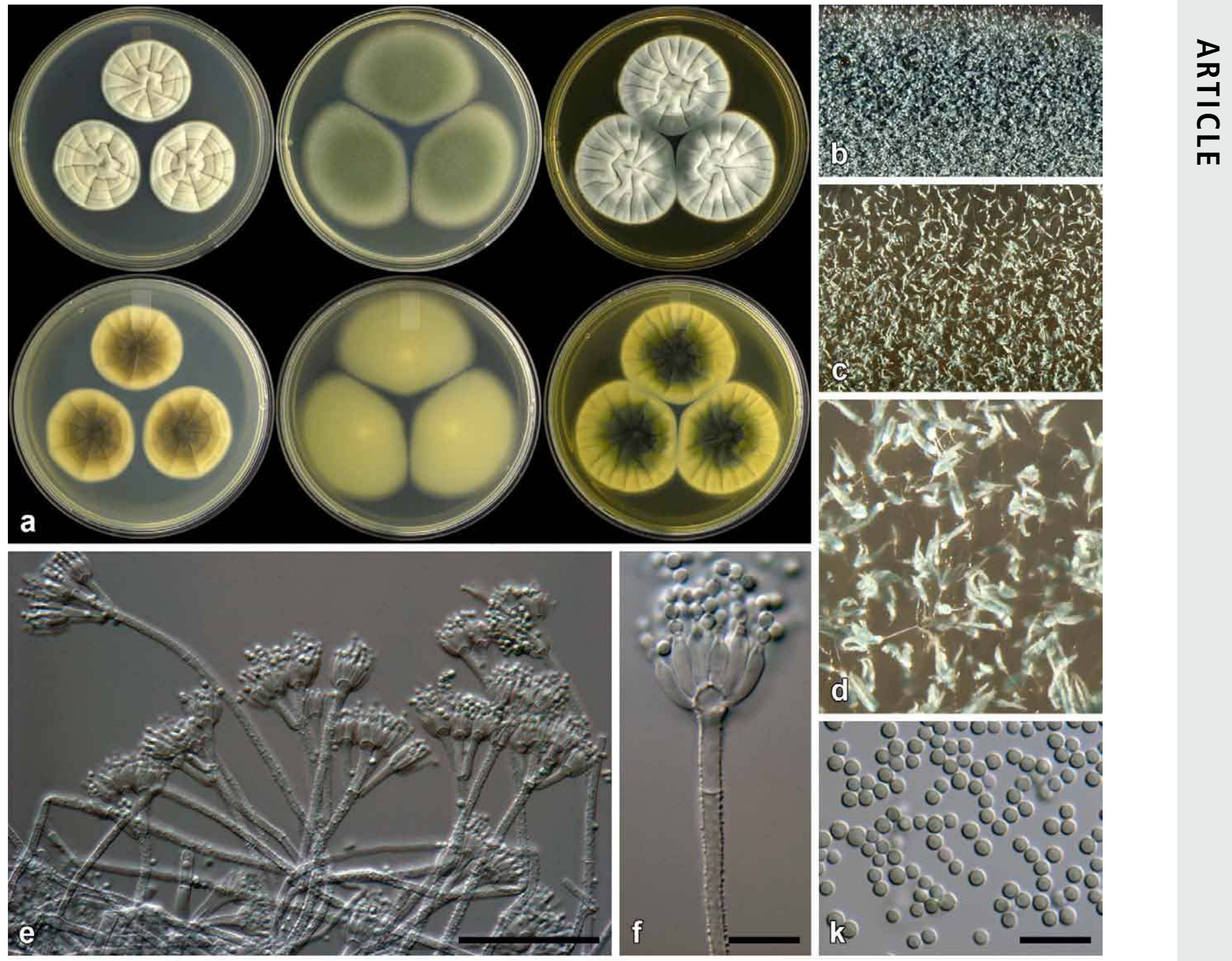

$3 x+1+4=2+\frac{1}{2}$

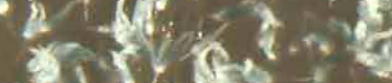

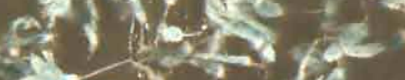

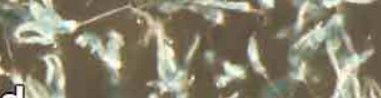

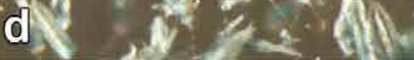

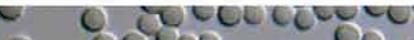
Q 9888800080 cos oog ofoc

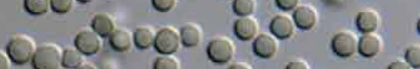
- 85 g o 80 d - 809 o 880 is oco gogos

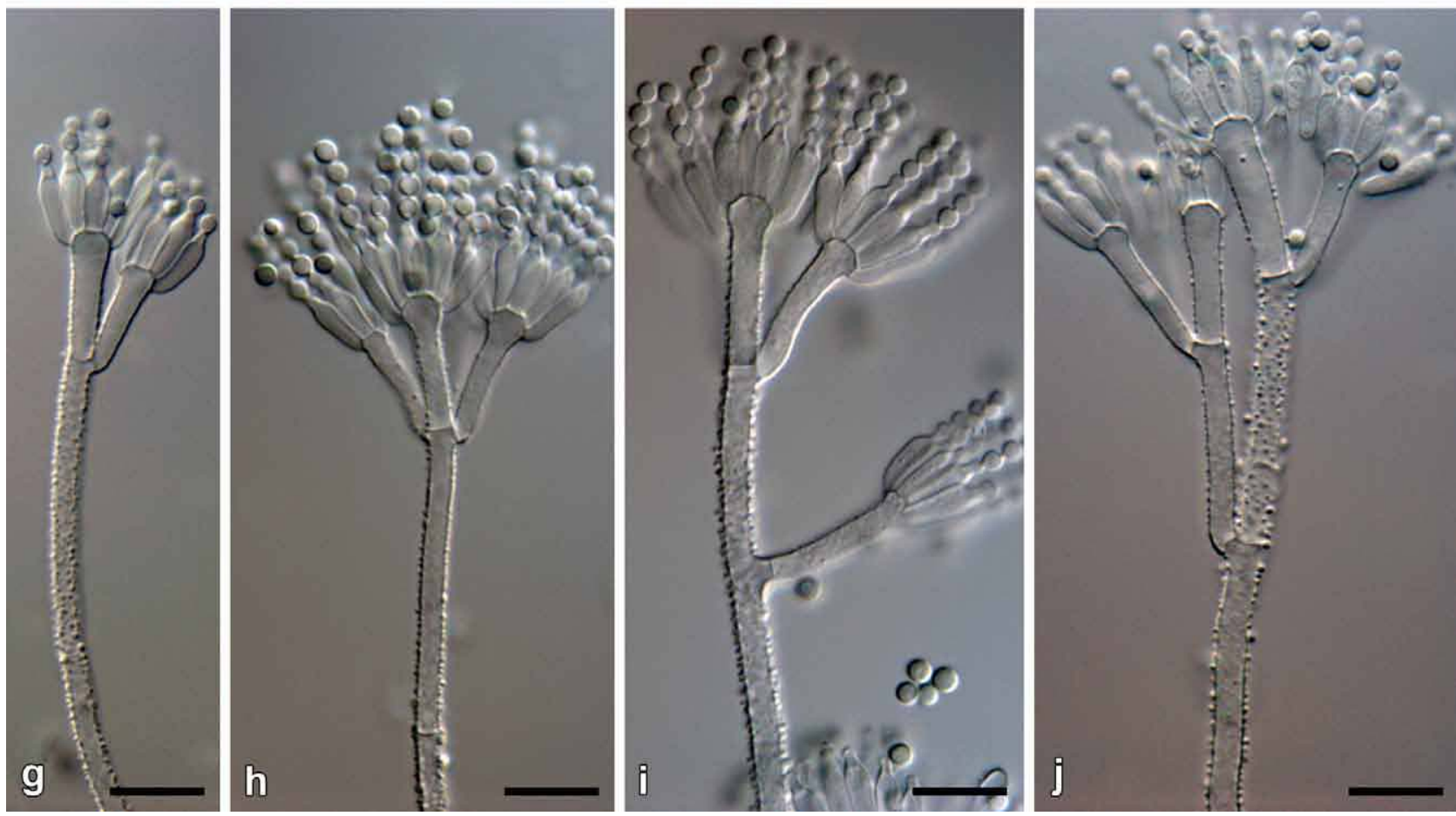

Fig. 10. Penicillium consobrinum (CBS 139144). a. Colonies (top row, left to right: CYA, MEA, YES; bottom row, left to right: CYA reverse, MEA reverse, YES reverse). b. Texture on CYA. c-d. Texture on MEA. e-j. Conidiophores. k. Conidia. Bars e $=50 \mu \mathrm{m}, \mathrm{f}-\mathrm{k}=10 \mu \mathrm{m}$. 

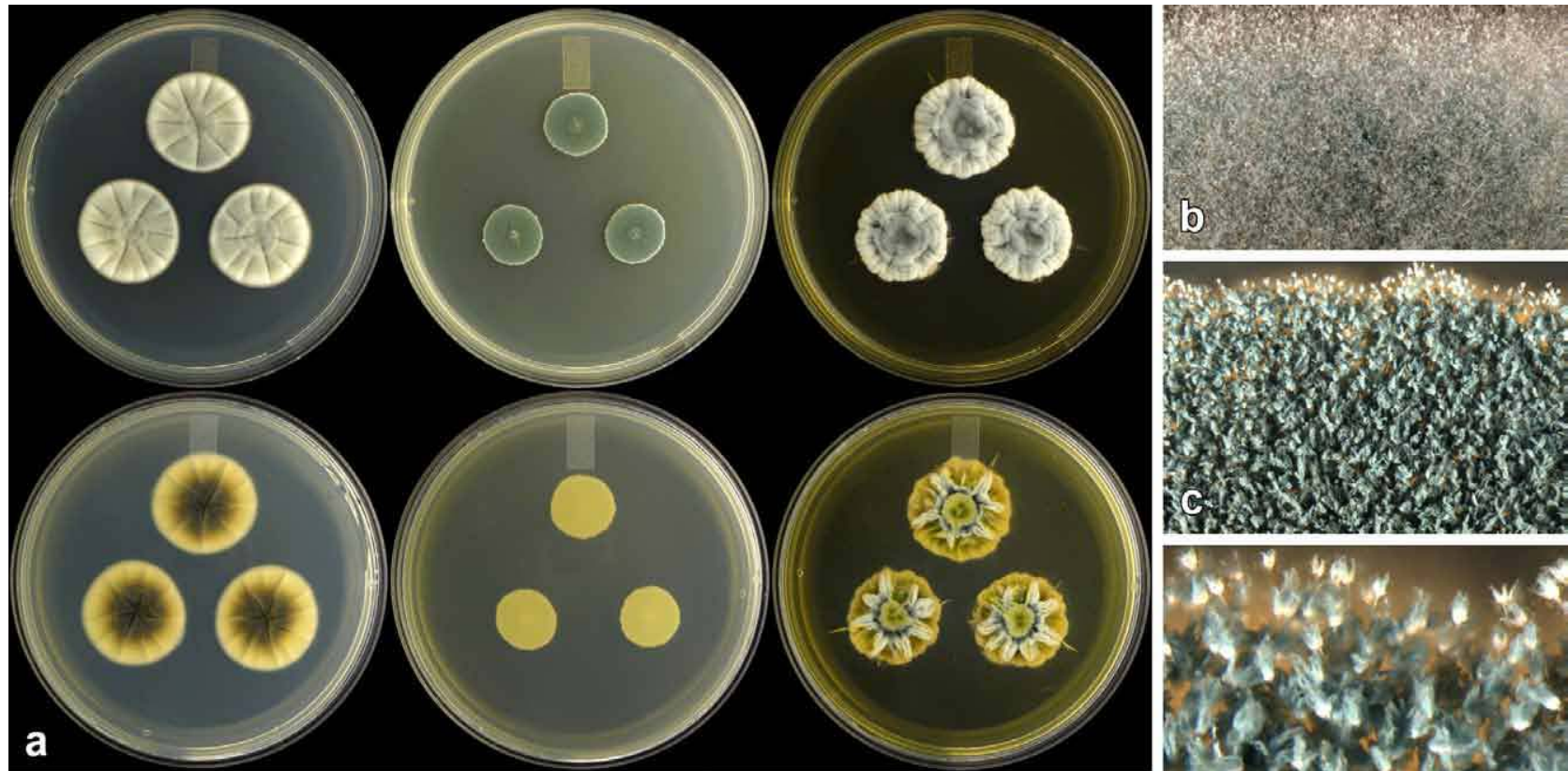

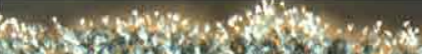

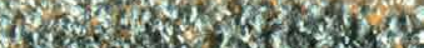

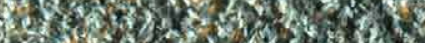

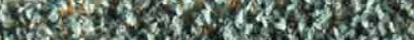

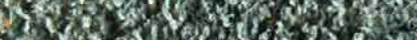
3. $\mathrm{s}$. G

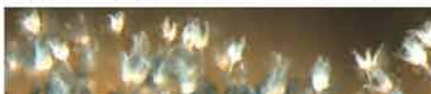

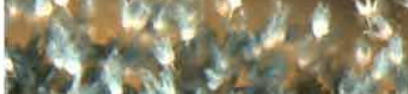

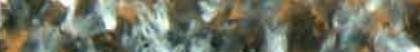

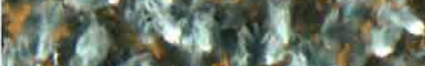

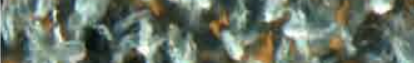

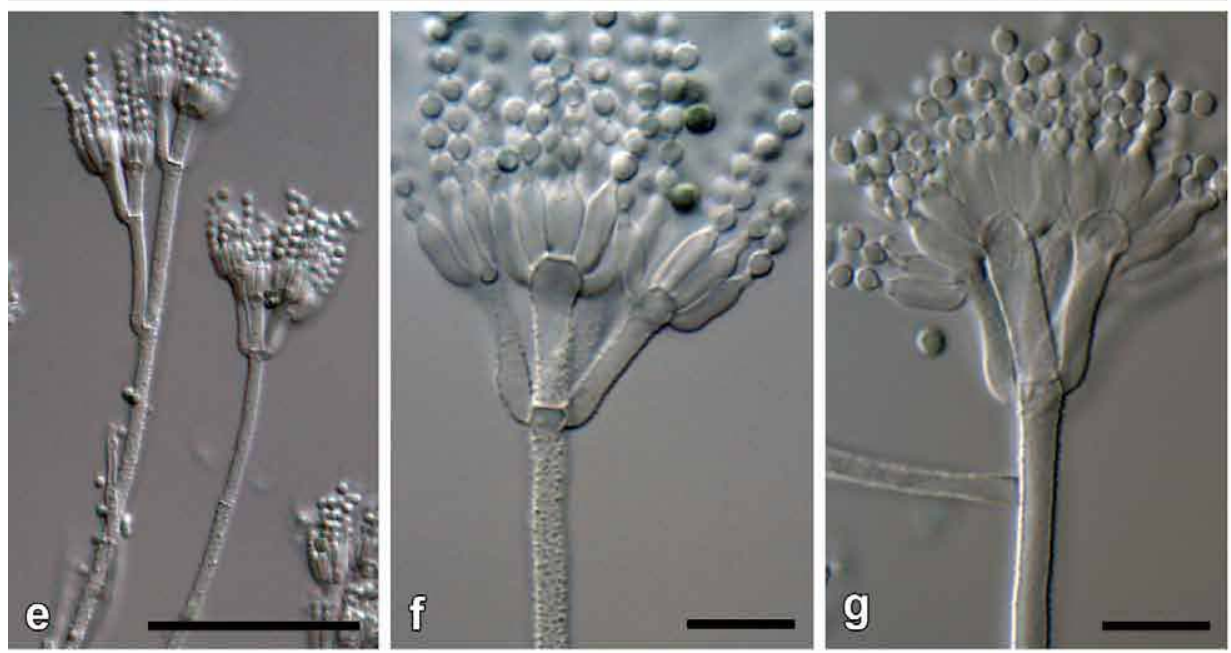

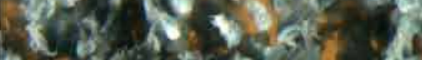

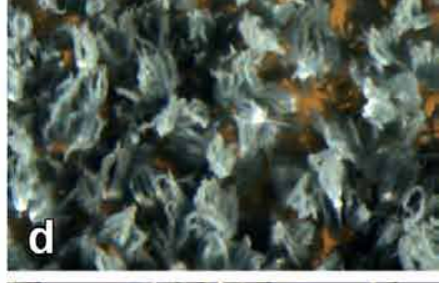

d $(2) \sqrt{2}$, and
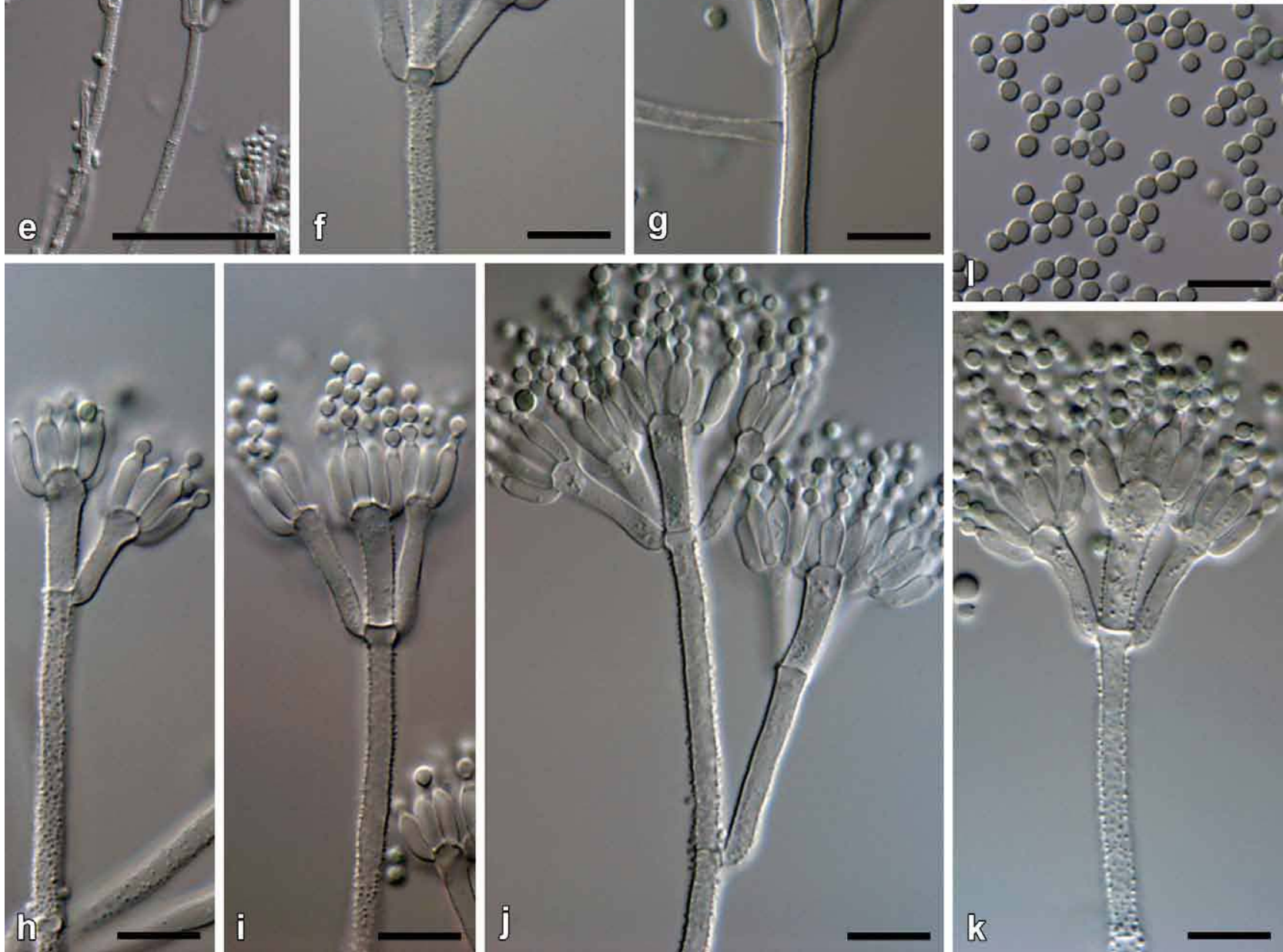

\section{()}

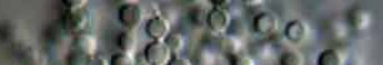

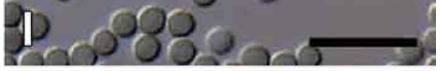

x)
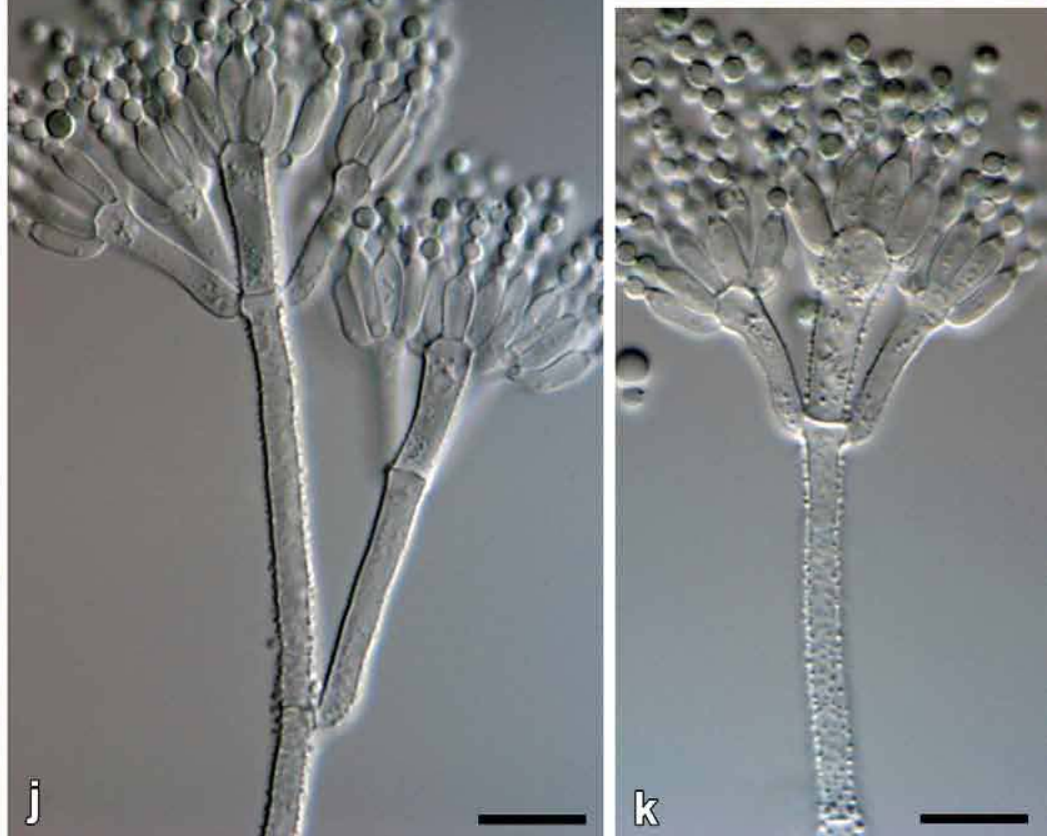

Fig. 11. Penicillium cravenianum (CBS 139138). a. Colonies (top row, left to right: CYA, MEA, YES; bottom row, left to right: CYA reverse, MEA reverse, YES reverse). b. Texture on CYA. c-d. Texture on MEA. e-k. Conidiophores. I. Conidia. Bars e $=50 \mu \mathrm{m}, \mathrm{f}-\mathrm{I}=10 \mu \mathrm{m}$. 
Colony characters: CYA, $25^{\circ} \mathrm{C}, 7 \mathrm{~d}$ : Colonies low to moderately deep, radially and concentrically sulcate; margins low, narrow, entire; mycelia white; texture velutinous; sporulation sparse to sometimes moderate, conidia en masse dull green (30E3-4) at centre, dull green to greyish green (25D4-5) elsewhere; exudate absent; soluble pigment absent; reverse dark green to olive (30F5-8-1F5-8) at centre, pale yellow (2A3-3A3) near edge. MEA, $25^{\circ} \mathrm{C}, 7 \mathrm{~d}$ : Colonies low, plane; margins subsurface, narrow, entire; mycelia white; texture velutinous, floccose near centre; sporulation dense, conidia en masse dark green (25F826F8); exudate absent; soluble pigment absent; reverse yellow (3B8) at centre, fading into dull yellow (3B4) margin. YES, 25 ${ }^{\circ} \mathrm{C}, 7 \mathrm{~d}$ : Colonies low, radially and concentrically sulcate, with randomly raised furrows; margins narrow, entire; mycelia white; texture floccose; sporulation sparse to sometimes moderately dense, conidia en masse similarly coloured as CYA; exudate absent; soluble pigment absent; reverse greyish green (28D6E6) at centre, light to greyish yellow (3A5-B5) at margin. CREA, $25^{\circ} \mathrm{C}, 7 \mathrm{~d}$ : Acid not produced.

Micromorphology: Conidiophores mostly biverticillate, some terverticillate; stipes rough, 120-325 × 2.5-4 $\mu \mathrm{m}$; Branches 2 when present, $16-48 \times 2.5-4 \mu \mathrm{m}$; Metulae $2-6$ per stipe, slightly divergent, $31-73^{\circ}(51 \pm 10.3), 11-22 \times 2.5-4.5 \mu \mathrm{m}$ $(15.4 \pm 2.1 \times 3.1 \pm 0.4)$, vesicle $3.5-5.5 \mu \mathrm{m}(4.7 \pm 0.5)$; Phialides ampulliform, 8-12 per metula, 7-9.5 × 2.5-3.5 $\mu \mathrm{m}(8.1 \pm 0.6$ $\times 3 \pm 0.2$ ); Conidia finely rough, globose to broadly ellipsoidal, $2.5-3 \times 2-3 \mu \mathrm{m}(2.6 \pm 0.2 \times 2.4 \pm 0.1)$, average width/length $=$ $0.93, n=112$.

Notes: Penicillium cravenianum characteristically produces colonies with restricted growth on MEA, somewhat resembling those of $P$. brevicompactum, and conidiophores with rough walls. These characters distinguish it from all close relatives in the $P$. corylophilum clade (Figs 3-4). Penicillium brevicompactum produces smooth walled conidiophores. See also under $P$. consobrinum.

\section{Penicillium hemitrachum Visagie \& K. Jacobs, sp. nov. \\ MycoBank MB811004}

(Fig. 12)

Etymology: Latin, meaning half rough walled, named in reference to the rough and smooth walled metulae produced on the same conidiophore.

Diagnosis: Differs from close relatives by producing fast growing colonies especially on CYA at $30{ }^{\circ} \mathrm{C}$; conidiophores have a brownish to green pigmentation, have rough walled stipes, while different metulae from the same conidiophore can be rough or smooth; conidia globose $2-2.5 \mu \mathrm{m}$.

Type: South Africa: Malmesbury, isol. ex air sample, Feb. 2007, isol. C. M. Visagie (CBS H-22042 [dried culture] holotype; CBS 139134 = DAOMC 241098 = DTO 180-D8 = CV 2845 - ex-type cultures).

ITS barcode: FJ231003. Alternative identification markers: BenA $=\mathrm{JX} 141048, \mathrm{CaM}=\mathrm{JX} 157526, \mathrm{RPB2}=\mathrm{KP} 064642$.
Colony diam, 7 d (in $\mathrm{mm}$ ): CYA 54-58; CYA $30{ }^{\circ} \mathrm{C}$ 60-62; CYA $37{ }^{\circ} \mathrm{C}$ 4-9; MEA 68-70; YES 68-70; G25N 10-18; CREA 20-25.

Colony characters: CYA, $25^{\circ} \mathrm{C}, 7 \mathrm{~d}$ : Colonies low, radially sulcate, concentrically sulcate in fresh cultures, grey sterile hyphae present covering some conidial areas; margins low, narrow, entire; mycelia white; texture velutinous, some floccose areas present; sporulation dense, conidia en masse dull to greyish green (26E3-6), lighter dull green (26D3) near margin; exudate absent; soluble pigment yellow sometimes inconspicuous; reverse olive (3F6-7) in central areas, dull green (28D4-E4) elsewhere. MEA, $25{ }^{\circ} \mathrm{C}, 7 \mathrm{~d}$ : Colonies $\mathrm{mm}$, low, plane; margins low to subsurface, wide; mycelia white; texture velutinous, some floccose mycelia present; sporulation dense, conidia en masse dull green to greyish green (28F4-F6); exudate absent; soluble pigment yellow; reverse greyish yellow (1B6) at point of inoculation, greyish green (1D6) elsewhere. YES, $25{ }^{\circ} \mathrm{C}, 7 \mathrm{~d}$ : Colonies low, randomly sulcate; margins low, narrow; mycelia white; texture velutinous, floccose mycelia present near centre; sporulation dense, conidia en masse similar to CYA; exudate absent; soluble pigment absent; reverse similar to CYA. CREA, 25 ${ }^{\circ} \mathrm{C}, 7 \mathrm{~d}$ : Acid not produced.

Micromorphology: Conidiophores mostly biverticillate, sometimes monoverticillate, having a brownish to green pigment, smooth and rough metulae commonly present on the same conidiophore; Stipes rough, conidiophores with short stipes often smooth, 20-180 × 2-3.5 $\mu \mathrm{m}$; Metulae 2-4 per stipe, divergent, $38-67^{\circ}(52 \pm 8.8), 9.5-18 \times 2-3.5 \mu \mathrm{m}(13.6 \pm 1.9 \times$ $2.9 \pm 0.3)$, vesicle $3-6 \mu \mathrm{m}(4.2 \pm 0.6)$; Phialides ampulliform, mostly 8-12 per metula, some only 4-6, 7-9 × 2-3.5 $\mu \mathrm{m}$ $(7.7 \pm 0.5 \times 2.8 \pm 0.2)$; Conidia finely rough, globose, $2-2.5 \times$ $2-2.5 \mu \mathrm{m}(2.4 \pm 0.1 \times 2.4 \pm 0.1)$, average width/length $=0.98$, $n=108$.

Notes: Penicillium hemitrachum produces fast growing colonies, especially on CYA at $30^{\circ} \mathrm{C}$, but grows restrictedly at $37^{\circ} \mathrm{C}$. Colony appearance on MEA can easily be confused with Aspergillus fumigatus. Conidiophores often have a green pigmentation and are borne from green mycelia. Interestingly, some conidiophores have smooth and rough walled metulae on the same conidiophore. Phylogenetically the new species is related to $P$. maclennaniae, $P$. smithii and $P$. velutinum (Fig. 2). The fast growth rate of $P$. hemitrachum, $P$. maclennaniae and $P$. velutinum distinguish them from $P$. smithii. However, $P$. maclennaniae produces conidiophores lacking pigment and produces larger conidia (3.8-4.0 $\mu \mathrm{m}$ fide Yip 1981) than $P$. hemitrachum, while $P$. velutinum has smooth non-pigmented stipes and grows slightly slower on CYA at $25^{\circ} \mathrm{C}$.

Penicillium momoii Visagie \& K. Jacobs, sp. nov. MycoBank MB811007

(Fig. 13)

Etymology: Latin, momoii, named for Momo the dog, from the hide and seek book Find Momo by Andrew Knapp (Knapp 2014), reflecting the challenge of locating a single specimen 

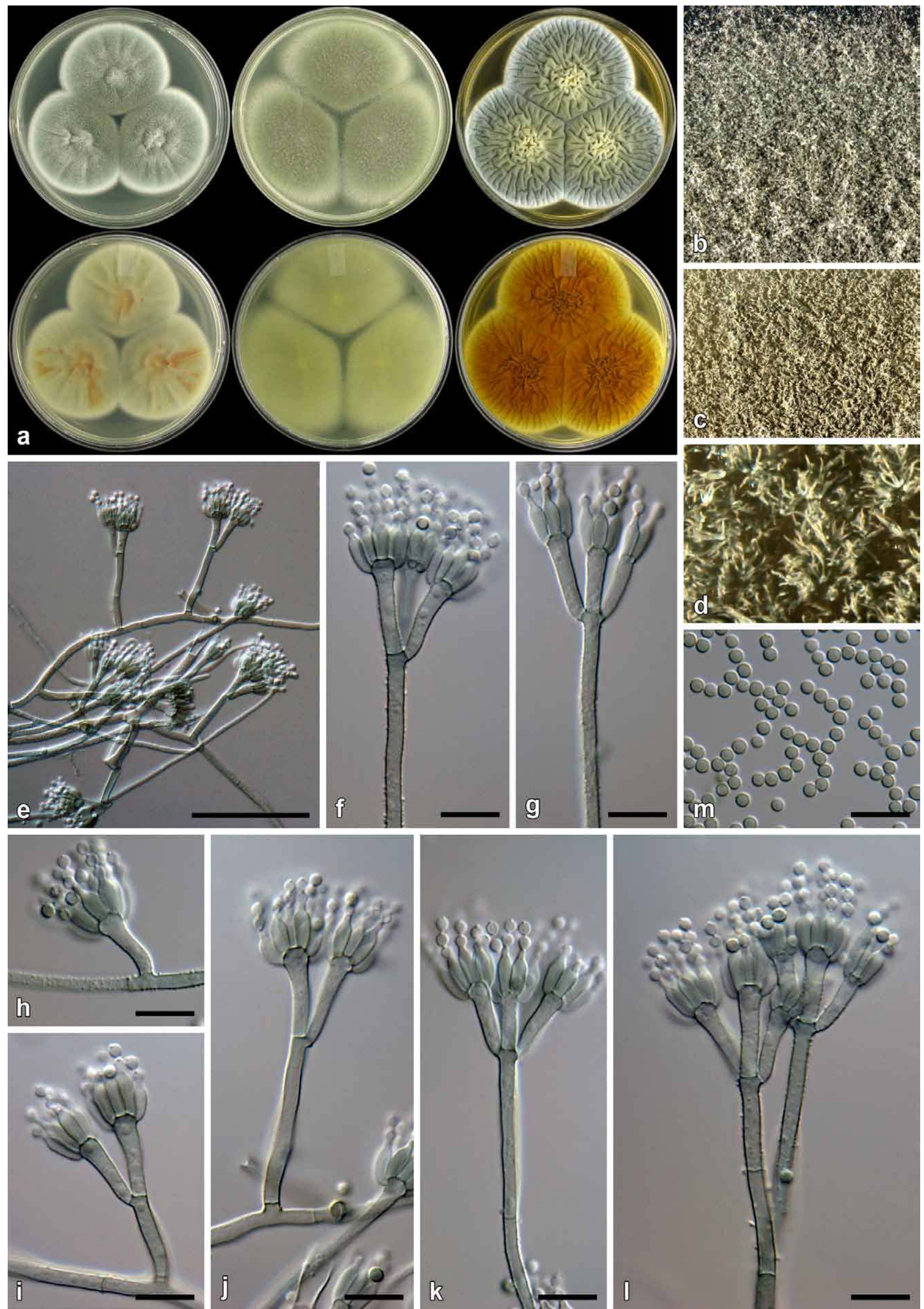

Fig. 12. Penicillium hemitrachum (CBS 139134). a. Colonies (top row, left to right: CYA, MEA, YES; bottom row, left to right: CYA reverse, MEA reverse, YES reverse). b. Texture on CYA. c-d. Texture on MEA. e-l. Conidiophores. $m$. Conidia. Bars e $=50 \mu \mathrm{m}, \mathrm{f}-\mathrm{m}=10 \mu \mathrm{m}$. 

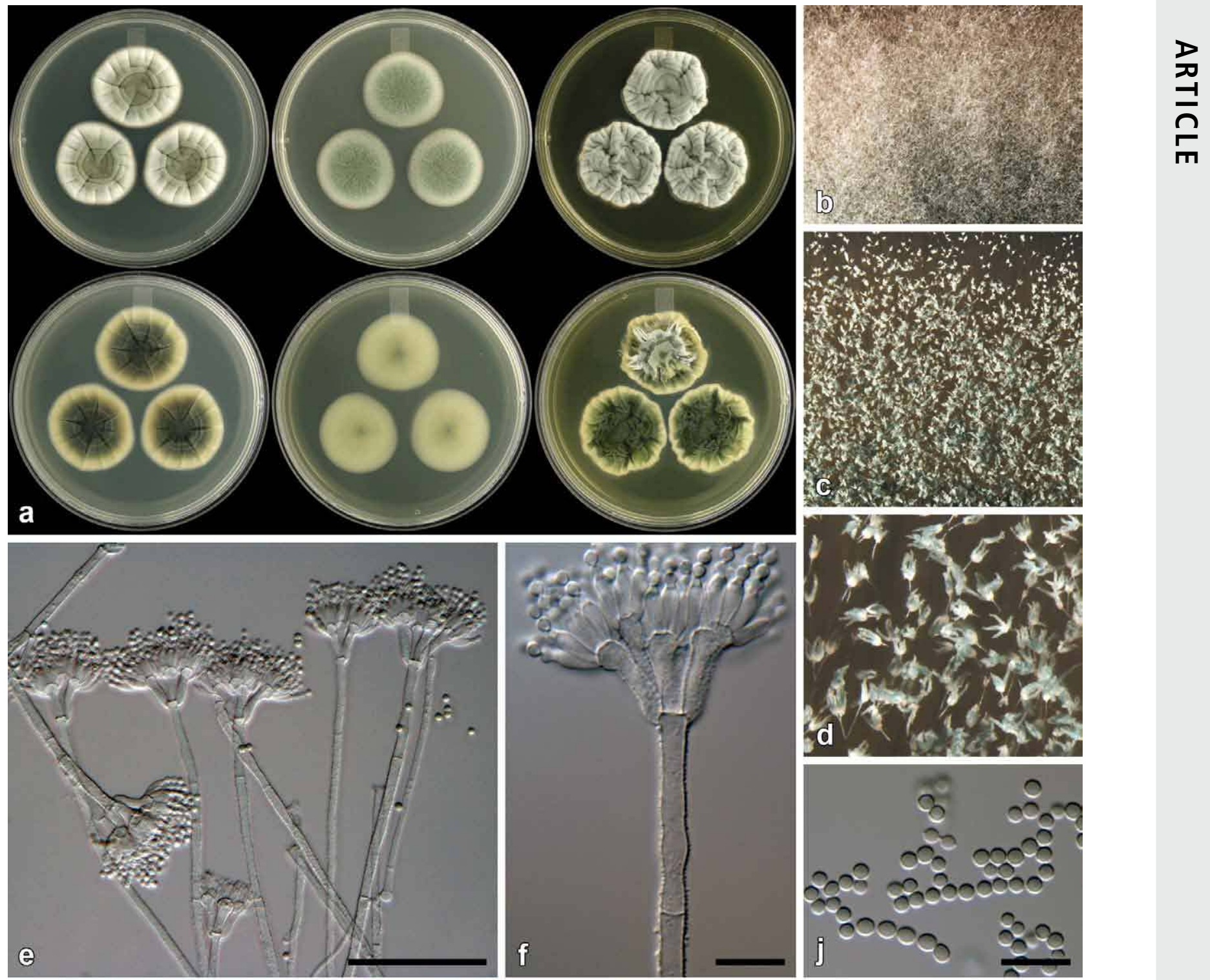

(b)
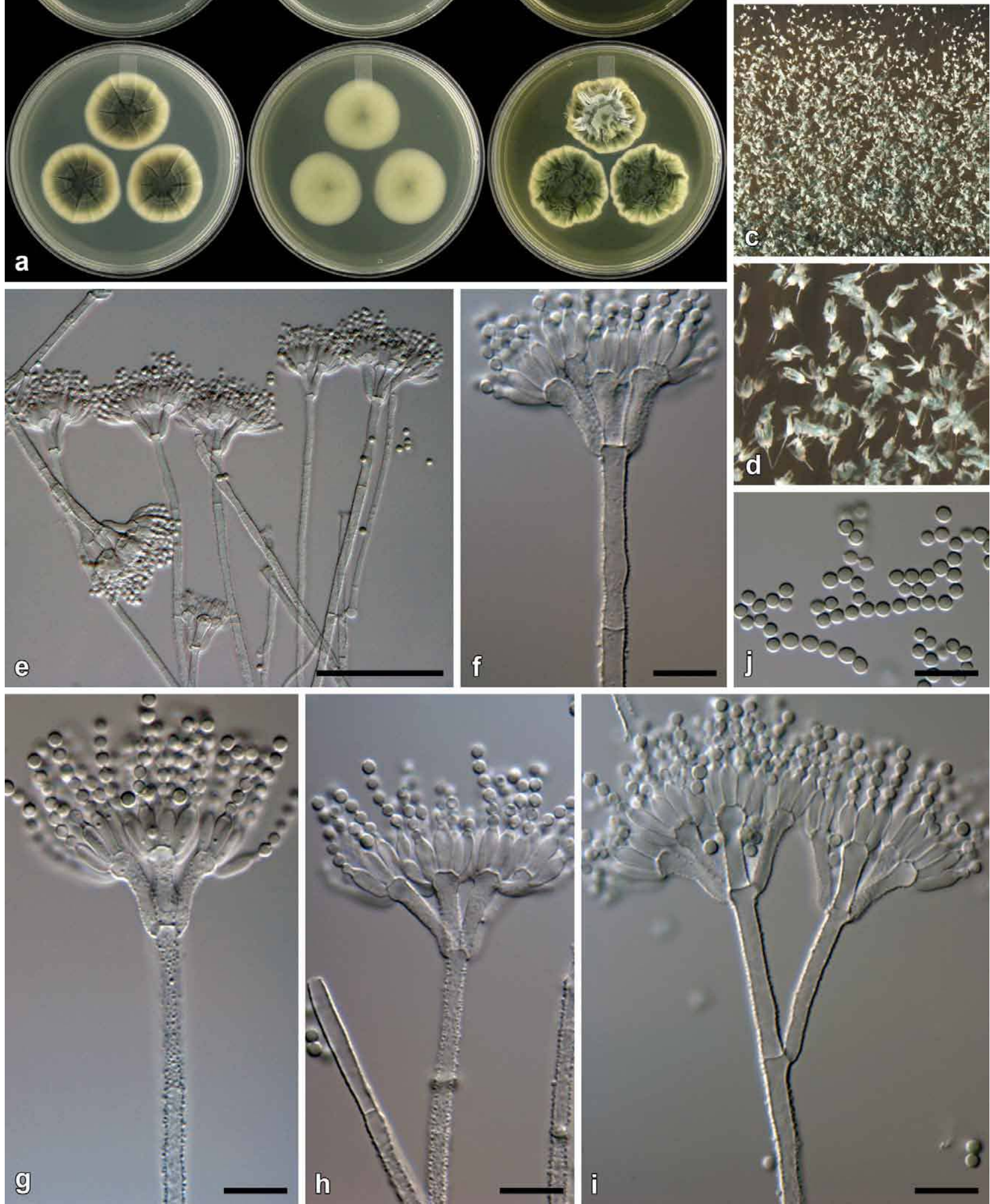

Fig. 13. Penicillium momoii (CBS 139157). a. Colonies (top row, left to right: CYA, MEA, YES; bottom row, left to right: CYA reverse, MEA reverse, YES reverse). b. Texture on CYA. c-d. Texture on MEA. e-i. Conidiophores. j. Conidia. Bars e $=50 \mu \mathrm{m}, \mathrm{f}-\mathrm{j}=10 \mu \mathrm{m}$. 
of a unique species among thousands of morphologically similar cultures.

Diagnosis: Differs from close relatives by being able to grow on CYA at $37^{\circ} \mathrm{C}(5-8 \mathrm{~mm})$, grows more restricted on MEA and $G 25 N$ than $P$. subturcoseum and $P$. repensicolum colonies sporulate much denser.

Type: South Africa: Malmesbury, isol. ex mite from Protea repens infructescence, Jun. 2009, isol. C. M. Visagie (CBS $\mathrm{H}-22046$ dried culture] - holotype; CBS 139157 = DAOMC 241077 = DTO 182-G4 = CV 1015 - ex-type cultures).

ITS barcode: JX140895. Alternative identification markers:

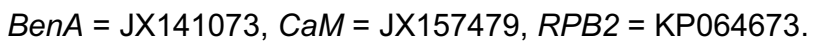

Colony diam, 7 d (in mm): CYA 30-32; CYA $30{ }^{\circ} \mathrm{C} 27-28$; CYA $37{ }^{\circ} \mathrm{C}$ 5-8; MEA 30-31; YES 28-31; G25N 15-16; CREA 15-17.

Colony characters: CYA $, 25^{\circ} \mathrm{C}, 7 \mathrm{~d}$ : Colonies low to moderately deep, radially and concentrically sulcate; margins low, narrow to wide, somewhat irregular; mycelia white; texture floccose; sporulation moderately dense at centre, conidia en masse greyish green (27E5-7); exudate absent; soluble pigment absent; reverse greyish to dark green (25E7-F7) at centre, greenish white (26A2-27A2) at margin. MEA, $25{ }^{\circ} \mathrm{C}, 7 \mathrm{~d}$ : Colonies low, plane; margins low, narrow, entire; mycelia white; texture velutinous, with floccose areas present; sporulation dense, conidia en masse greyish green (27E57); exudate absent; soluble pigment absent; reverse greyish to dull yellow (2B3-3B3) at centre, dull green (28D4-30D4) elsewhere. YES, $25^{\circ} \mathrm{C}, 7 \mathrm{~d}$ : Colonies low to moderately deep, radially and concentrically sulcate, random furrows also present; margins low, narrow, entire; mycelia white; texture velutinous, some floccose areas; sporulation ranging from moderately sparse to moderately dense at centre, conidia en masse greyish green (27E5-7); exudate absent; soluble pigment absent; reverse pigmentation greyish green to dark green (26E7-28F7-8) at centre, pale yellow (2A3) at margin. CREA, $25^{\circ} \mathrm{C}, 7 \mathrm{~d}$ : Acid not produced.

Micromorphology: Conidiophores biverticillate, a minor proportion monoverticillate, terverticillate not common; Stipes rough walled to warted, $100-400 \times 2.5-3.5 \mu \mathrm{m}$; Branches 2 when present, $20-30 \times 2.5-3.5 \mu \mathrm{m}$; Metulae 2-5 per stipe, divergent, sometimes slightly appressed, $41-102^{\circ}(64 \pm 14), 12-19 \times 2.5-3.5$ $\mu \mathrm{m}(14.8 \pm 1.6 \times 3.1 \pm 0.2)$, vesicle $4.5-6 \mu \mathrm{m}(5.1 \pm 0.4)$; Phialides ampulliform, 10-12 per metula, 7.5-9.5 × 2.5-3 $\mu \mathrm{m}(8.3 \pm 0.5$ $\times 2.8 \pm 0.2)$; Conidia rough, globose, $2-3 \times 2-3 \mu \mathrm{m}(2.5 \pm 0.1 \times$ $2.5 \pm 0.1$ ), average width/length $=0.98, n=31$.

Notes: See under P. consobrinum.

Penicillium pagulum Visagie \& K. Jacobs, sp. nov. MycoBank MB811005

(Fig. 14)

Etymology: Latin, pagulum, small village; in reference to the small colonies produced on CYA at $25^{\circ} \mathrm{C}$.
Diagnosis: Differs from close relatives by its restricted growth on CYA at $25^{\circ} \mathrm{C}(15-20 \mathrm{~mm})$.

Type: South Africa: Struisbaai, isol. ex bract from Protea repens infructescence, Aug. 2009, isol. C. M. Visagie (CBS $\mathrm{H}-22049$ [dried culture] - holotype; CBS 139166 = DAOMC 241069 = DTO 183-H2 = CV 2224 - ex-type cultures).

ITS barcode: JX140898. Alternative identification markers: BenA $=\mathrm{JX} 141070, \mathrm{CaM}=\mathrm{JX} 157519, \mathrm{RPB2}=\mathrm{KP} 064655$.

Colony diam, 7 d (in mm): CYA 15-20; CYA $30{ }^{\circ} \mathrm{C} \mathrm{12-15;}$ CYA $37{ }^{\circ} \mathrm{C}$ 1-5; MEA 25-32; YES 26-34; G25N 5-10; CREA 19-24.

Colony characters: CYA, $25{ }^{\circ} \mathrm{C}, 7 \mathrm{~d}$ : Colonies low, radially sulcate; margins low, narrow, entire; mycelia white; texture velutinous, some floccose areas; sporulation sparse, conidia en masse greyish turquoise (24B5-E5); exudate absent; soluble pigment absent; reverse olive (2F5-3F5). MEA, $25{ }^{\circ} \mathrm{C}, 7 \mathrm{~d}$ : Colonies low, plane; margins subsurface, wide, entire; mycelia white; texture velutinous; sporulation dense, conidia en masse greyish to dark green (25E5-F5); exudate absent; soluble pigment absent; reverse greyish yellow (3B7) at centre, greyish green $(30 \mathrm{C} 5)$ at margin. YES, $25^{\circ} \mathrm{C}, 7 \mathrm{~d}$ : Colonies low, radially and concentrically sulcate; margins low, narrow, entire; mycelia white; texture velutinous, some floccose areas present; sporulation moderately dense, conidia en masse greyish turquoise (24B3) in some regions, greyish turquoise (24E5) in others; exudate absent; soluble pigment absent; reverse dark green (25F8), orange (6B7) at margin. CREA, $25^{\circ} \mathrm{C}, 7 \mathrm{~d}$ : Acid not produced.

Micromorphology: Conidiophores biverticillate, a minor proportion terverticillate; Stipes finely rough, 100-250 × 2.5$4 \mu \mathrm{m}$; Branches 2 when present, $21-32 \times 2.5-4 \mu \mathrm{m}$; Metulae 2-5 per stipe, divergent, $28-62^{\circ}(41 \pm 8.4), 13-24 \times 2.5-4 \mu \mathrm{m}$ $(16.8 \pm 2.5 \times 3.1 \pm 0.4)$, vesicle $3.5-5 \mu \mathrm{m}(4.3 \pm 0.5)$; Phialides ampulliform, 9-14 per metula, 8-10.5 × 2.5-3.5 $\mu \mathrm{m}(9.3 \pm 0.6$ $\times 3 \pm 0.2$ ); Conidia finely rough, globose to subglobose, $2-3 \times$ $2-3 \mu \mathrm{m}(2.6 \pm 0.2 \times 2.5 \pm 0.2)$, average width/length $=0.97, n$ $=35$.

Notes: Penicillium pagulum displays restricted growth on CYA, which distinguishes it from all phylogenetically closely related species (Figs 3-4). See also under P. consobrinum.

Penicillium repensicola Visagie \& K. Jacobs, sp. nov. MycoBank MB811006

(Fig. 15)

Etymology: Latin, repensicola, dwelling in "repens"; in reference to the type strain, which was isolated from Protea repens.

Diagnosis: Differs from close relatives by being able to grow on CYA at $37^{\circ} \mathrm{C}(7-15 \mathrm{~mm})$, faster than its closest relatives $P$. momoii and $P$. subturcoseum. Colonies also sporulate much denser than the latter two. 

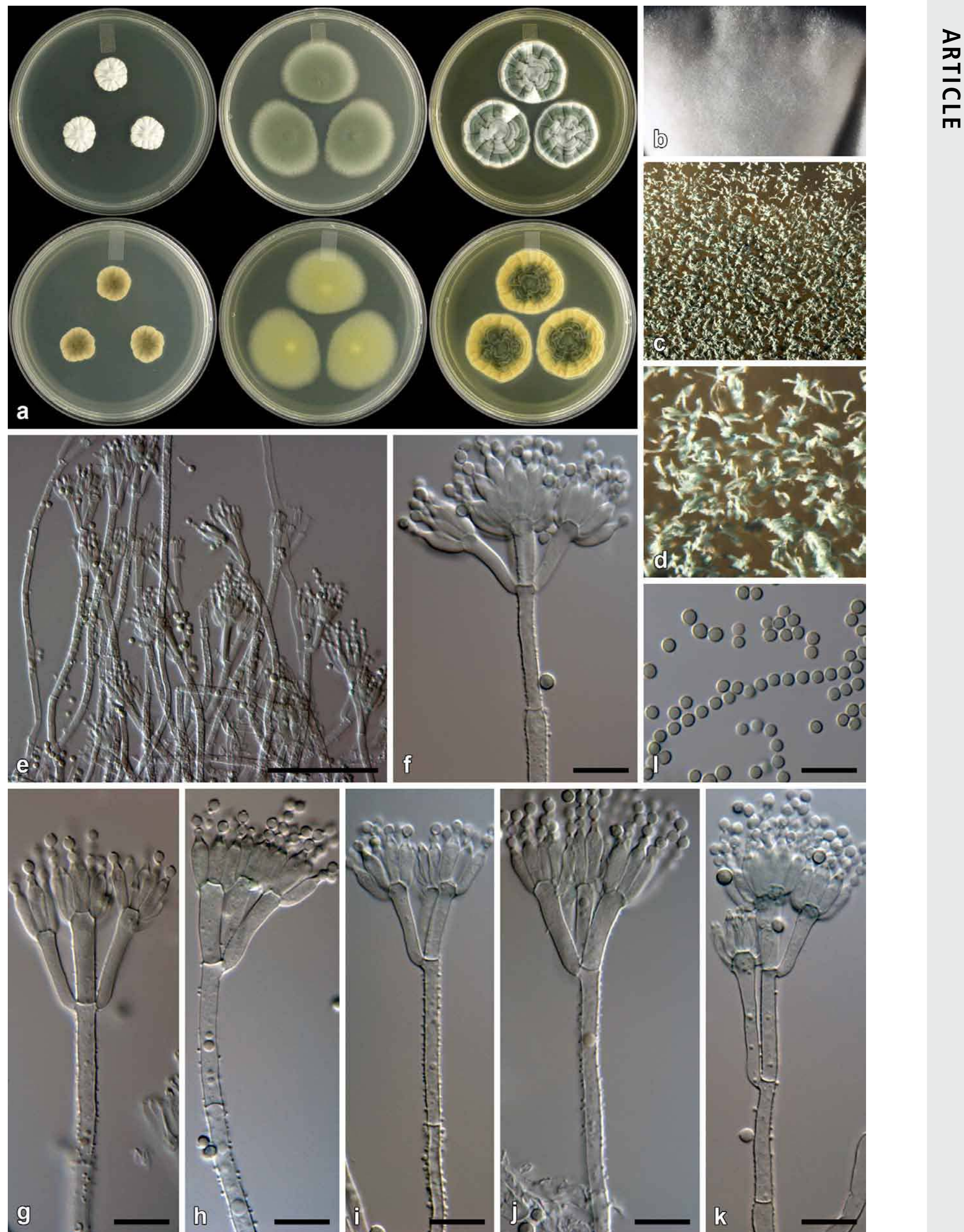

(6)

م
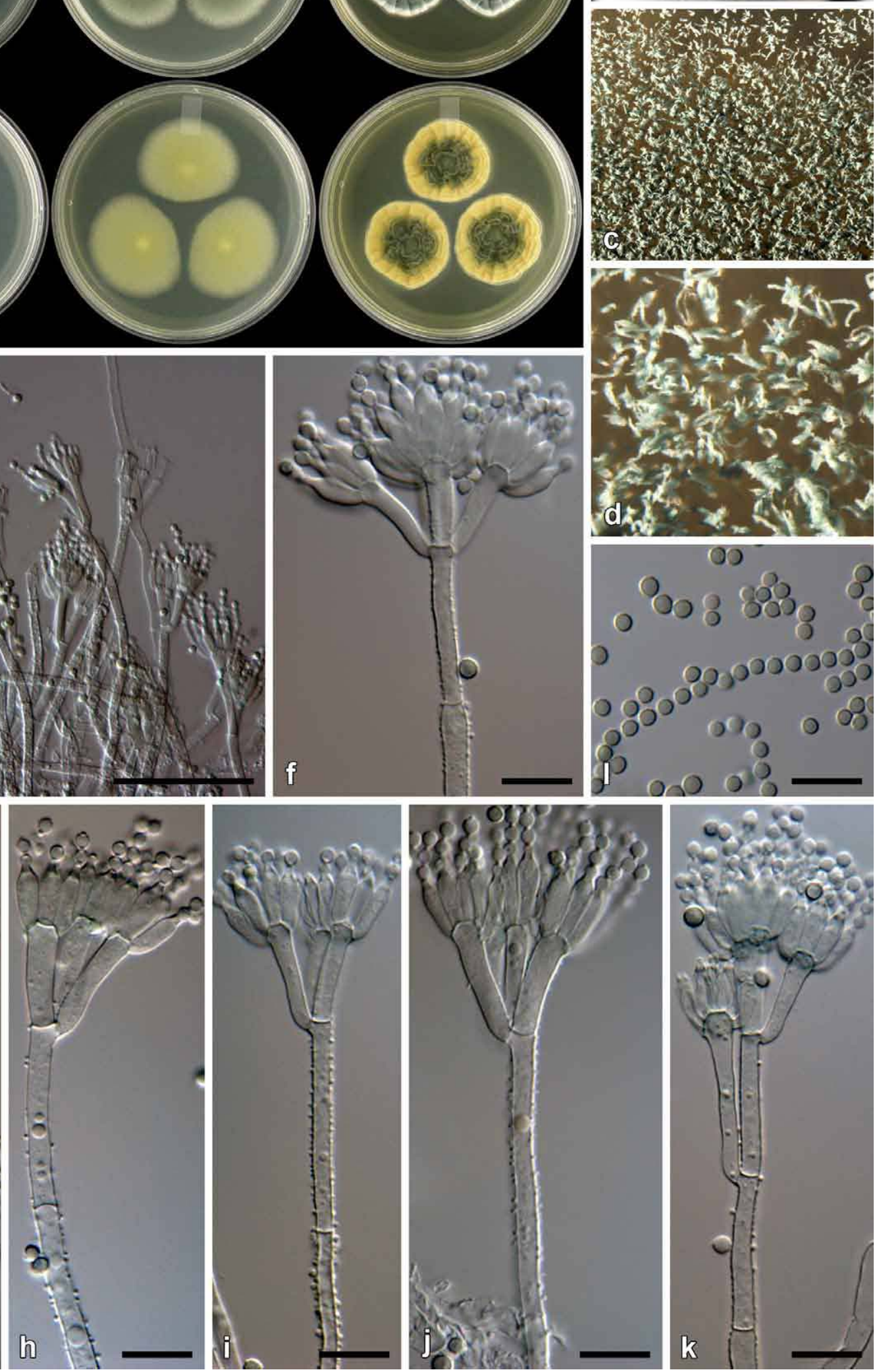

Fig. 14. Penicillium pagulum (CBS 139166). a. Colonies (top row, left to right: CYA, MEA, YES; bottom row, left to right: CYA reverse, MEA reverse, YES reverse). b. Texture on CYA. c-d. Texture on MEA. e-k. Conidiophores. I. Conidia. Bars e $=50 \mu \mathrm{m}, \mathrm{f}-\mathrm{I}=10 \mu \mathrm{m}$. 

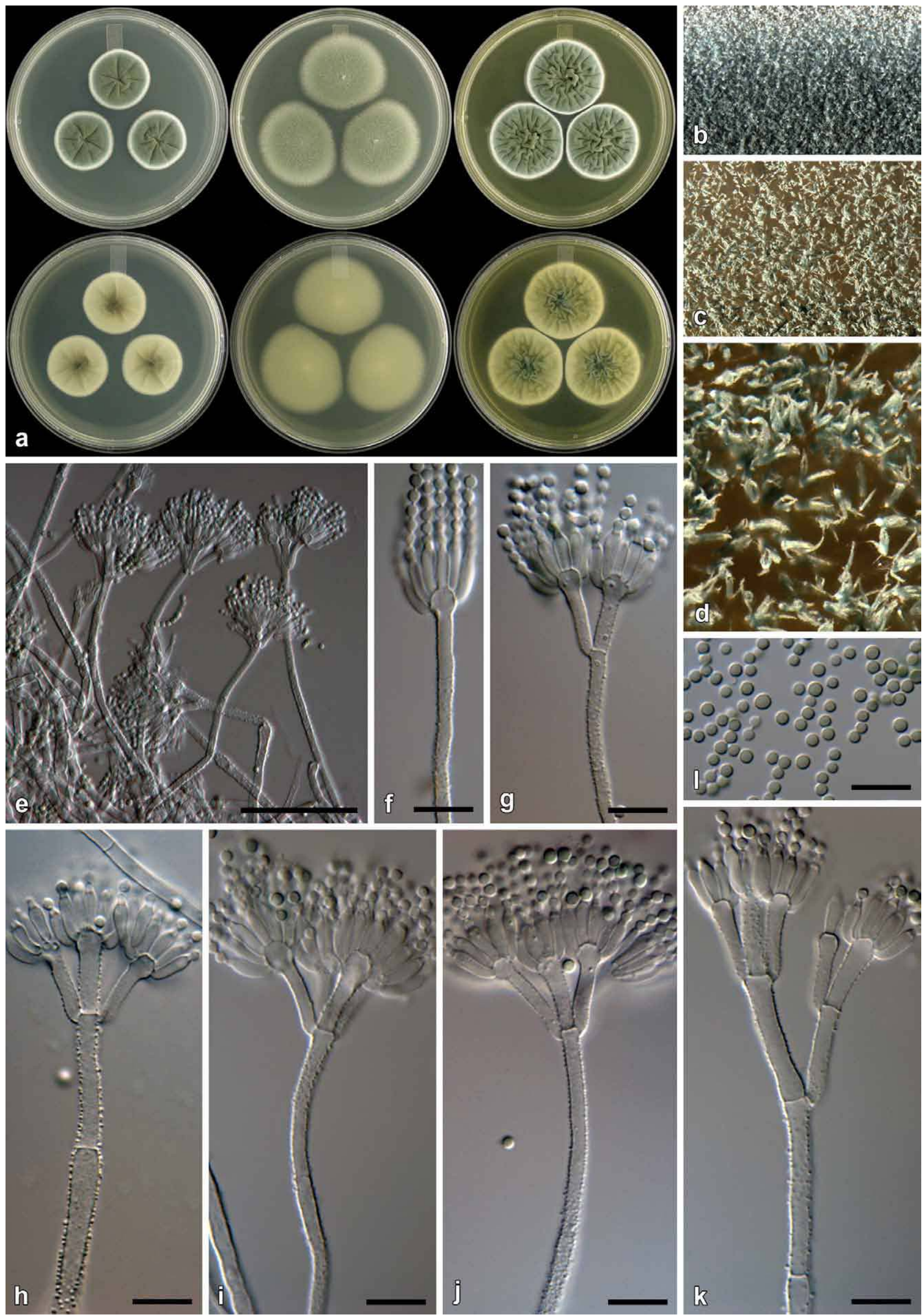

Fig. 15. Penicillium repensicola (CBS 139160). a. Colonies (top row, left to right: CYA, MEA, YES; bottom row, left to right: CYA reverse, MEA reverse, YES reverse). b. Texture on CYA. c-d. Texture on MEA. e-k. Conidiophores. I. Conidia. Bars e $=50 \mu \mathrm{m}, \mathrm{f}-\mathrm{I}=10 \mu \mathrm{m}$. 
Type: South Africa: Malmesbury, isol. ex bract from Protea repens infructescence, Jun. 2009, isol. C. M. Visagie (CBS $\mathrm{H}-22047$ [dried culture] - holotype; CBS 139160 = DAOMC 241080 = DTO 183-B8 = CV 1495 - ex-type cultures).

ITS barcode: JX140893. Alternative identification markers:

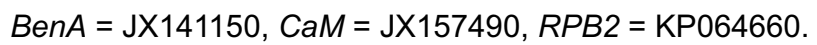

Colony diam, 7 d (in mm): CYA 23-32; CYA $30{ }^{\circ} \mathrm{C}$ 20-28; CYA $37{ }^{\circ} \mathrm{C}$ 7-15; MEA 30-43; YES 28-38; G25N 10-22; CREA 15-17.

Colony characters: CYA $, 25^{\circ} \mathrm{C}, 7 \mathrm{~d}$ : Colonies low to moderately deep, radially and concentrically sulcate; margins low, narrow to wide, entire; mycelia white; texture velutinous; sporulation moderately dense, conidia en masse dark green (25F726F7); exudate absent; soluble pigment absent; reverse dark green to olive green (30F8-3F8) at centre, greenish white (26A2-27A2) at margin. MEA, $25^{\circ} \mathrm{C}, 7 \mathrm{~d}$ : Colonies low, plane; margins low, narrow, entire; mycelia white; texture velutinous, floccose areas present; sporulation dense, conidia en masse greyish green (27E5-7); exudate absent; soluble pigment absent; reverse sometimes olive brown (4F8) and sometimes greyish to dull yellow (2B3-3B3) at centre, dull green (28D430D4) elsewhere. YES, $25^{\circ} \mathrm{C}, 7 \mathrm{~d}$ : Colonies low to moderately deep, radially and concentrically sulcate, random furrows also present; margins low, narrow, entire; mycelia white; texture velutinous and floccose; sporulation moderately dense, conidia en masse dark green (25F7-26F7); exudate absent; soluble pigment absent; reverse pigmentation greyish to dark green (26E7-28F7-8) at centre, pale yellow (2A3) at margin. CREA, $25^{\circ} \mathrm{C}, 7 \mathrm{~d}$ : Acid not produced.

Micromorphology: Conidiophores biverticillate, a minor proportion monoverticillate, terverticillate not common; Stipes rough walled to warted, $80-260 \times 2.5-3.5 \mu \mathrm{m}$; Branches 2 when present, $17-26 \times 2.5-3.5 \mu \mathrm{m}$; Metulae $2-5$ per stipe, divergent, sometimes slightly appressed, 50-92 $(65 \pm 10)$, $13-22 \times 2.5-3.5 \mu \mathrm{m}(16 \pm 1.9 \times 3 \pm 0.3)$, vesicle 4-6 $\mu \mathrm{m}(5 \pm 0.6)$; Phialides ampulliform, 10-12 per metula, 7-10 × 2.5-3.5 $\mu \mathrm{m}$ $(8.5 \pm 0.6 \times 2.8 \pm 0.3)$; Conidia rough, globose, $2-3 \times 2-3 \mu \mathrm{m}$ $(2.4 \pm 0.2 \times 2.4 \pm 0.2)$, average width/length $=0.97, n=37$.

Notes: See under $P$. consobrinum.

\section{Penicillium subturcoseum Visagie \& K. Jacobs, sp. nov. \\ MycoBank MB811008}

(Fig. 16)

Etymology: Latin, subturcoseum, turquoise beneath; in reference to the colony reverse colour on CYA.

Diagnosis: Differs from close relatives by being able to grow on CYA at $37{ }^{\circ} \mathrm{C}(1-2 \mathrm{~mm})$, even though growth is more restricted at this temperature than $P$. momoii and $P$. repensicola. Also, $P$. repensicola colonies sporulate much denser than the other two, while $P$. subturcoseum colonies show more restricted growth on MEA and G25N than $P$. momoii.
Type: South Africa: Malmesbury, isol. ex soil sample, Feb. 2007, isol. C. M. Visagie (CBS H-22041 [dried culture] holotype; culture ex-type CBS $139132=$ DAOMC $241096=$ DTO 180-C9 = CV 2835 ex-type cultures).

ITS barcode: FJ231006. Alternative identification markers: $B e n A=\mathrm{JX} 141161, \mathrm{CaM}=\mathrm{JX} 157532, \mathrm{RPB2}=\mathrm{KP} 064674$.

Colony diam, 7 d (in mm): CYA 29-39; CYA $30{ }^{\circ} \mathrm{C}$ 20-28; CYA $37{ }^{\circ} \mathrm{C}$ 1-2; MEA 34-43; YES 28-38; G25N 18-22; CREA 15-17.

Colony characters: CYA, $25^{\circ} \mathrm{C}, 7 \mathrm{~d}$ : Colonies low, radially and concentrically sulcate; margins low, narrow, somewhat irregular; mycelia white; texture velutinous, floccose areas present; sporulation moderately dense, conidia en masse greyish green (25E6); exudate absent; soluble pigment absent; reverse dark turquoise (24F7) to dark green (25F7). MEA, $25^{\circ} \mathrm{C}, 7 \mathrm{~d}$ : Colonies low, plane; margins low, narrow, entire; mycelia white; texture velutinous; sporulation moderately dense to dense, conidia en masse greyish to dark green (25E5-F8); exudate absent; soluble pigment absent; reverse greenish grey (29B2), sometimes dark green (25F8). YES, $25^{\circ} \mathrm{C}, 7 \mathrm{~d}$ : Colonies low to moderately deep, radially and concentrically sulcate, random furrows present; margins low, narrow, entire; mycelia white; texture velutinous and floccose; sporulation ranging from moderately sparse to moderately dense, conidia en masse similarly as CYA; exudate absent; soluble pigment absent; reverse greyish green to dark green (26E7-28F7-8) at centre, pale yellow (2A3) at margin. CREA, $25^{\circ} \mathrm{C}, 7 \mathrm{~d}$ : Acid not produced.

Micromorphology: Conidiophores biverticillate, a minor proportion monoverticillate, terverticillate not common; Stipes rough walled to warted, $45-350 \times 2.5-3.5 \mu \mathrm{m}$; Branches 2 when present, $20-30 \times 2.5-3.5 \mu \mathrm{m}$; Metulae 2-5 per stipe, divergent, sometimes slightly appressed, $27-100^{\circ}(58.7 \pm 15)$, $12-21 \times 2.5-4 \mu \mathrm{m}(16 \pm 1.8 \times 3 \pm 0.39)$, vesicle $4-6.5 \mu \mathrm{m}$ (5.1 \pm 0.7$)$; Phialides ampulliform, 10-12 per metula, 7-9.5 $\times$ 2.5-3.5 $\mu \mathrm{m}(8.3 \pm 0.7 \times 2.9 \pm 0.3)$; Conidia rough, globose, $2-3$ $\times 2-3 \mu \mathrm{m}(2.4 \pm 0.2 \times 2.4 \pm 0.2)$, average width/length $=0.97$, $n=69$.

Notes: See under $P$. consobrinum.

Penicillium xanthomelinii Visagie \& K. Jacobs, sp. nov.

MycoBank MB811009

(Fig. 17)

Etymology: Latin, xanthomelinii, xantho- yellow; in reference to the yellow colony reverse and the phylogenetic placement as a close relative of $P$. melinii.

Diagnosis: Differs from close relative, P. melinii, by faster colony growth on most media, production of yellow soluble pigments on CYA and lacking brown colours in colonies common in P. melinii. 

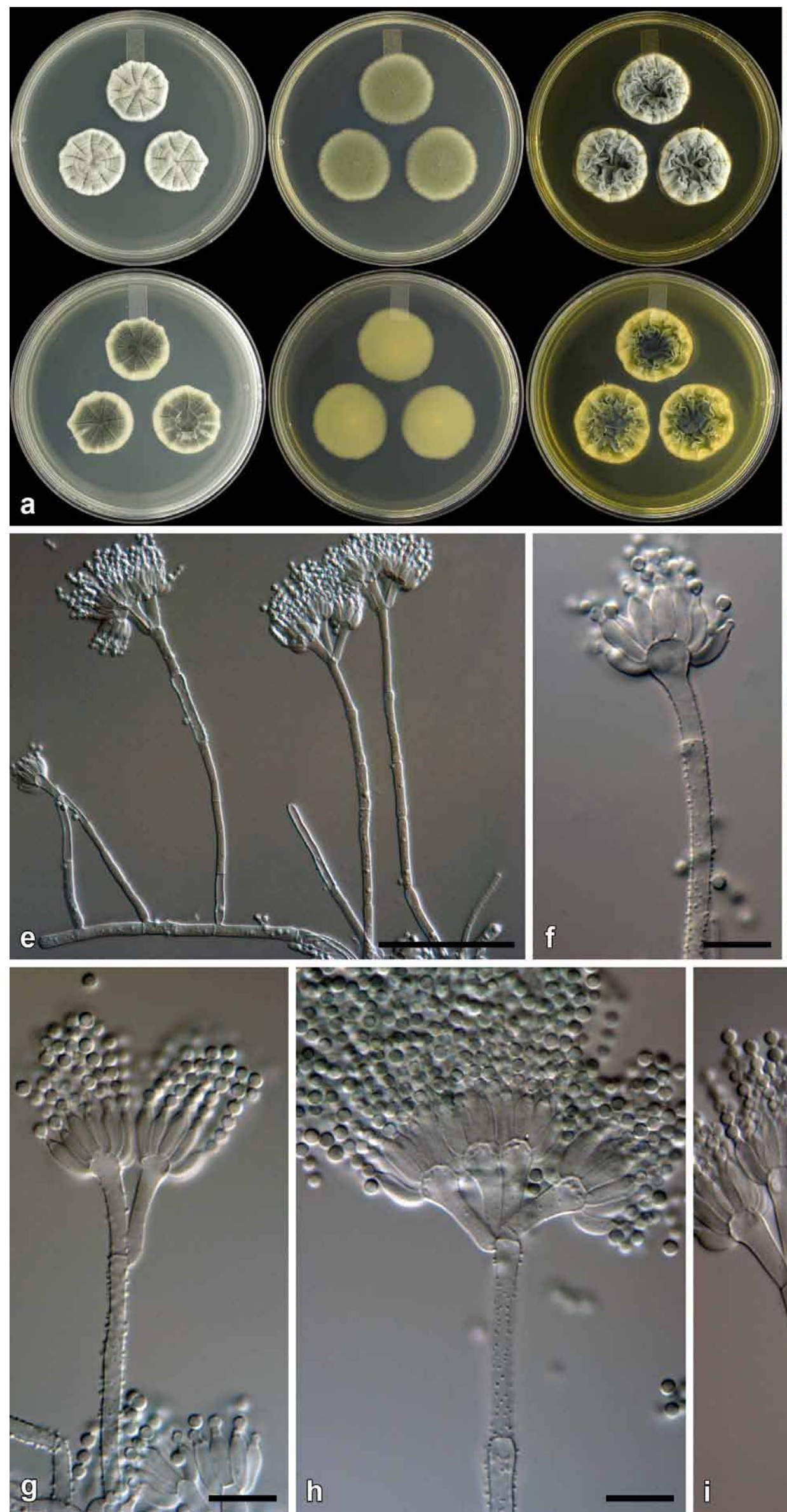
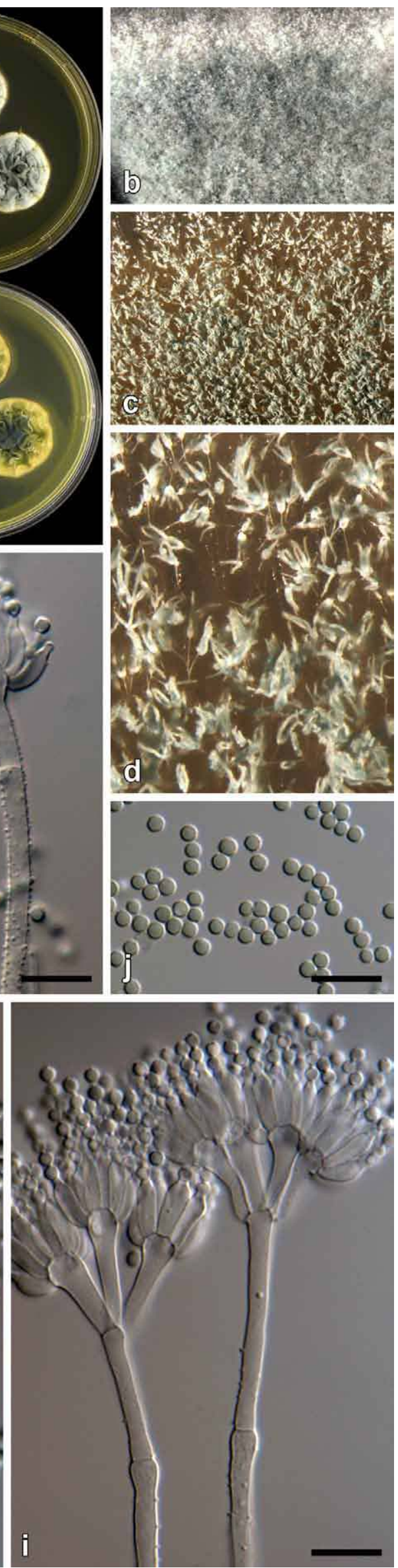

Fig. 16. Penicillium subturcoseum (CBS 139132). a. Colonies (top row, left to right: CYA, MEA, YES; bottom row, left to right: CYA reverse, MEA reverse, YES reverse). b. Texture on CYA. c-d. Texture on MEA. e-i. Conidiophores. j. Conidia. Bars e $=50 \mu \mathrm{m}, \mathrm{f}-\mathrm{j}=10 \mu \mathrm{m}$. 

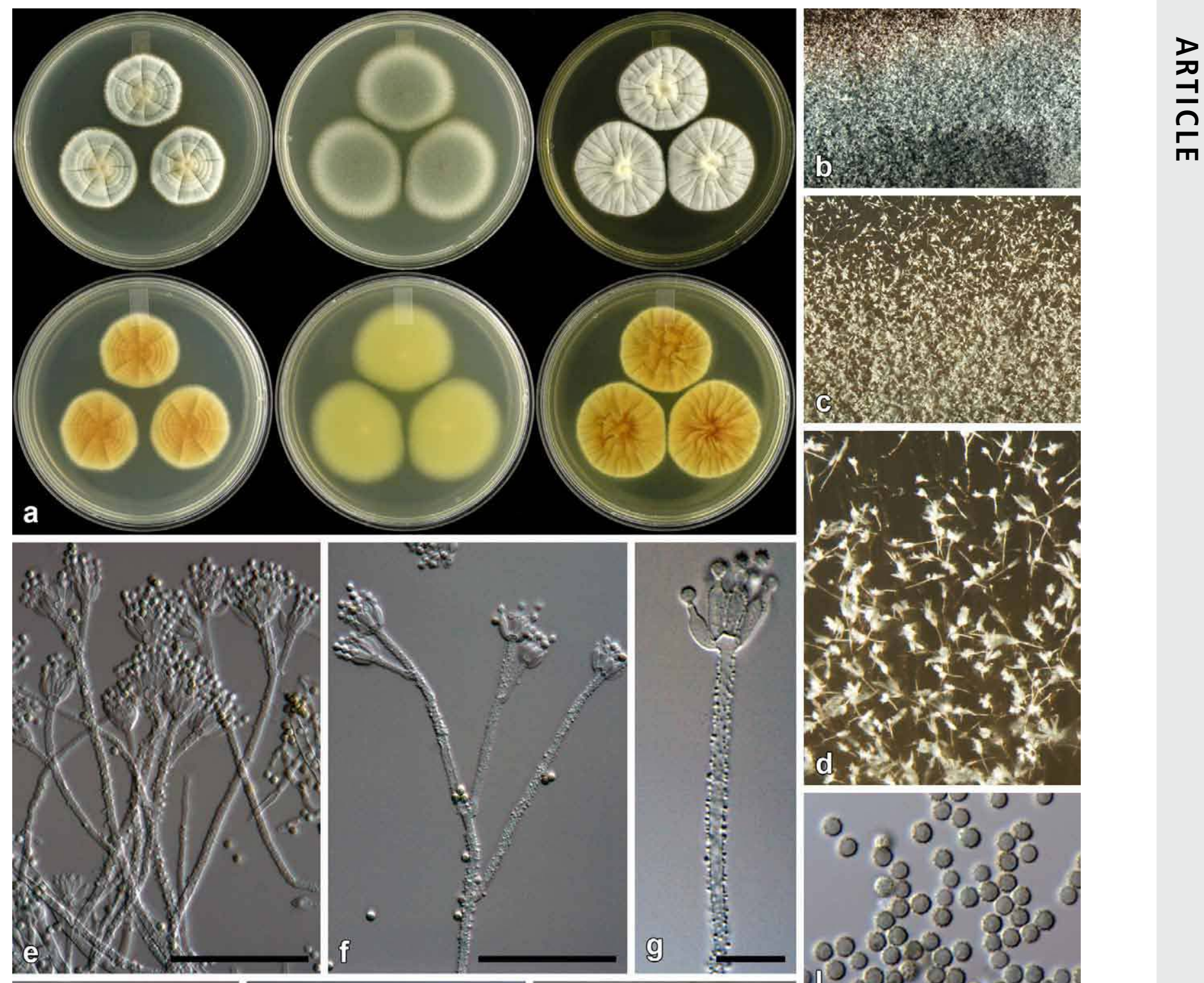

סִ

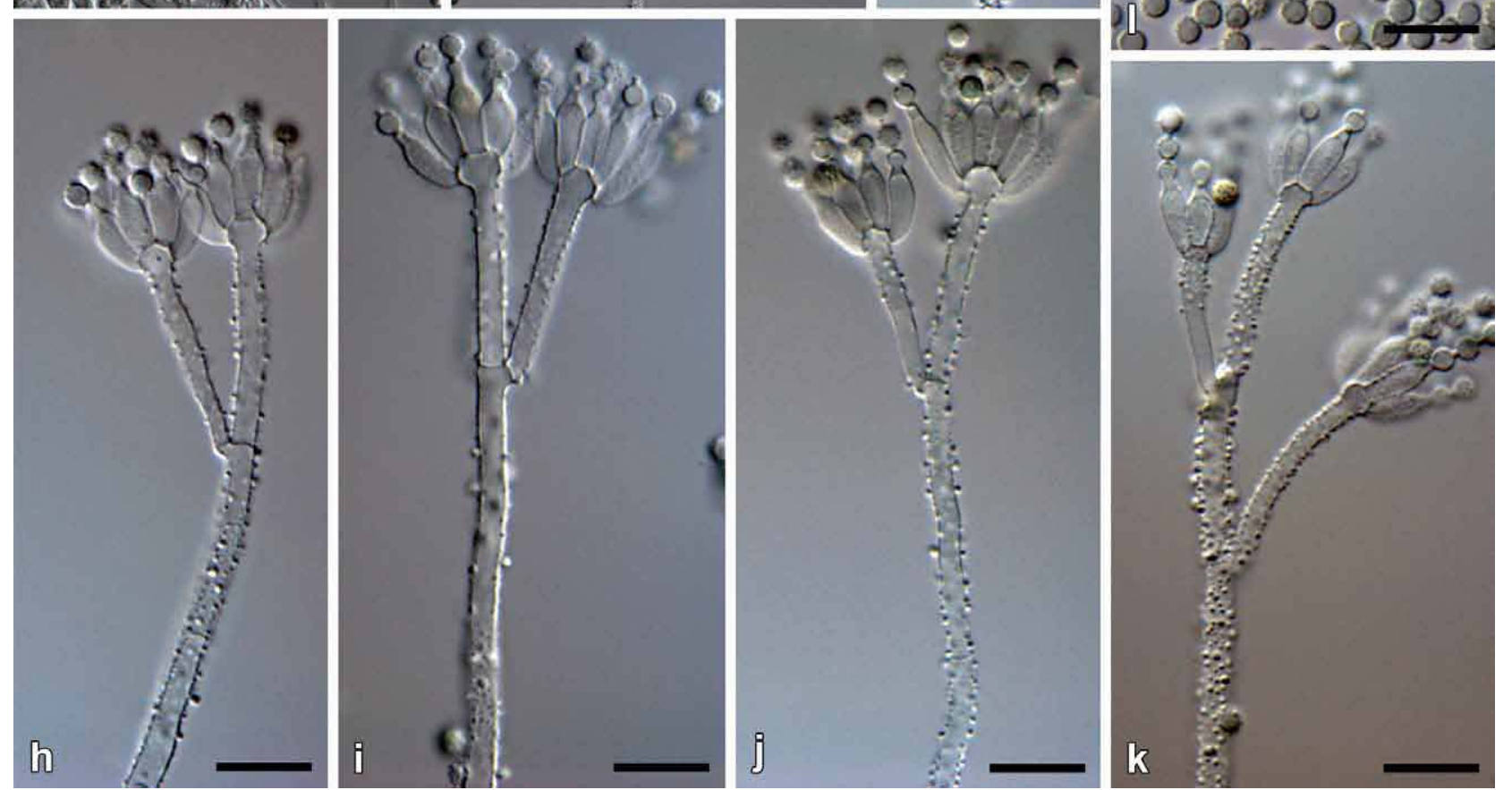

Fig. 17. Penicillium xanthomelinii (CBS 139163). a. Colonies (top row, left to right: CYA, MEA, YES; bottom row, left to right: CYA reverse, MEA reverse, YES reverse). b. Texture on CYA. c- d. Texture on MEA. e-k. Conidiophores. I. Conidia. Bars e-f $=50 \mu \mathrm{m}, g-I=10 \mu \mathrm{m}$. 
Type: South Africa: Struisbaai, isol. ex soil sample, Aug. 2009, isol. C. M. Visagie (CBS H-22048 [dried culture] holotype; CBS 139163 = DAOMC 241104 = DTO 183-C7 = CV 1677 - ex-type cultures).

ITS barcode: JX140921. Alternative identification markers: BenA $=\mathrm{JX} 141120, \mathrm{CaM}=\mathrm{JX} 157495, \mathrm{RPB2}=\mathrm{KP} 064683$

Colony diam, 7 d (in mm): CYA 30-33; CYA $30{ }^{\circ} \mathrm{C} 17-21$; CYA $37{ }^{\circ} \mathrm{C}$ no growth; MEA 39-45; YES 31-38; G25N 7-10; CREA 18-20.

Colony characters: CYA, $25{ }^{\circ} \mathrm{C}, 7 \mathrm{~d}$ : Colonies low, radially and concentrically sulcate; margins low, narrow, entire; mycelia white; texture velutinous at margin, floccose areas at centre; sporulation moderately dense, conidia en masse greyish dark green (24E5-F5), and various shades of greyish green (24D5-25B3-C3); exudate deep yellow to orange-brown, less pronounced in some isolates; soluble pigment deep yellow to orange-brown, inconspicuous; reverse brown (7E7-8) at colony centre, greyish orange to orange (5C5-8) elsewhere, greyish yellow (2B3-C3) near the yellowish white (2A2) margin. MEA, $25{ }^{\circ} \mathrm{C}, 7 \mathrm{~d}$ : Colonies low, plane; margins low, moderately wide, entire; mycelia white; texture velutinous, floccose areas present; sporulation moderately dense to dense, conidia en masse greyish green (25E5-F4-5); exudate absent; soluble pigment absent; reverse light yellow (3A5) at centre, greyish green (1C6-D6-7) elsewhere, margin greyish yellow (1B3). YES, $25^{\circ} \mathrm{C}, 7 \mathrm{~d}$ : Colonies low moderately deep near centre, radially and concentrically sulcate, random furrows also present; margins low, narrow, entire; mycelia white; texture velutinous, floccose areas present at centre; sporulation moderately dense, conidia en masse dull green (27E3-4), in less dense sporulating regions greyish green (27C2-3); exudate absent in most isolates, some with minute yellow to orange-brown droplets; soluble pigment absent; reverse golden yellow to orange (5B7-8) at centre, orange-yellow (4B8) elsewhere, becoming yellow (3B6) at margin. CREA, $25^{\circ} \mathrm{C}, 7 \mathrm{~d}$ : Acid not produced.

Micromorphology: Conidiophores biverticillate, subterminal monoverticillate branches common; Stipes rough walled to warted, $80-350 \times 2-3 \mu \mathrm{m}$; Branches 2 when present, divergent, 25-85 × 2-3 $\mu \mathrm{m}$; Metulae 2-4 per stipe, divergent, 20-67 (36.5 \pm 8.9$), 14-31 \times 2-3 \mu \mathrm{m}(20.9 \pm 3.7 \times 2.5 \pm 0.3$, vesicle $3-5 \mu \mathrm{m}(4 \pm 0.5)$; Phialides ampulliform, sometimes roughened, 8-14 per metula, often rough walled, 6.5-10 $\times$ 2.5-3.5 $\mu \mathrm{m}(8.3 \pm 0.7 \times 3 \pm 0.3)$; Conidia spinose, globose, $2.5-3 \times 2.5-3 \mu \mathrm{m}(2.8 \pm 0.1 \times 2.8 \pm 0.1$, average width/length $=0.98, n=61$.

Notes: Penicillium xanthomelinii characteristically produces rough walled to warted, divergent and irregular conidiophores, and commonly also has rough walled phialides and spinose conidia. Penicillium melinii, also isolated in this study, is the closest relative. However, $P$. melinii grows more restrictedly on most media. In addition, $P$. melinii produces brown colours in colonies, which are absent in $P$. xanthomelinii, while the latter produces yellow soluble pigments on CYA. Phylogenetically, P. xanthomelinii strains form a coherent group separate from $P$. melinii and its previously assigned synonyms (Fig. 2).

\section{Penicillium sect. Exilicaulis: accepted species and synonyms}

The following list includes accepted Penicillium sect. Exilicaulis species and their synonyms based on our findings in this study. For each entry we include (in order): (1) reference to original description; (2) MycoBank number associated with the name; (3) type specimen information; (4) living ex-type cultures available in various collections; and (5) GenBank accession numbers to reference sequences obtained from ex-type cultures. Accepted species are indicated by bold text. Synonyms are included in regular text below appropriate accepted names. The list also includes Eupenicillium (teleomorphic/sexual state) names that in all cases were introduced based on the same type strain as that of the Penicillium (anamorphic/asexual state) name, often both introduced in the same paper. Because these names refer to different morphs of the same specimen, they refer in principle to different parts of that specimen. We indicate these cases with "p.p." added after relevant herbarium accession numbers. Here we also correct typifications for $P$. cinereoatrum and $P$. guttulosum.

Citromyces griseus: See under Penicillium restrictum. Eupenicillium alutaceum: See under Penicillium alutaceum. Eupenicillium catenatum: See under Penicillium catenatum. Eupenicillium erubescens: See under Penicillium erubescens. Eupenicillium katangense: See under Penicillium katangense. Eupenicillium lapidosum: See under Penicillium lapidosum. Eupenicillium Iuzoniacum: See under Penicillium terrenum. Eupenicillium meridianum: See under Penicillium meridianum. Eupenicillium nepalense: See under Penicillium nepalense. Eupenicillium parvum: See under Penicillium parvum.

Eupenicillium papuanum: See under Penicillium parvum. Eupenicillium philippinense: See under Penicillium philippinense. Eupenicillium rubidurum: See under Penicillium rubidurum. Eupenicillium terrenum: See under Penicillium terrenum. Geosmithia namyslowskii: See under Penicillium namyslowskii. Penicillium aeneum: See under Penicillium citreonigrum.

Penicillium albocinerascens: See under Penicillium chalabudae.

Penicillium alutaceum D.B. Scott, Mycopathol. Mycol. Appl. 36: 17 (1968). [MB335708]. — Herb.: CBS 317.67 p.p. (holotype). Ex-type cultures: CBS 317.67 = ATCC 18542 = FRR 1158 = IFO $31728=$ IMI 136243. ITS barcode: AF033454. (Alternative markers: BenA = KJ834430; RPB2 = JN121489; CaM = KP016768).

Synonym: Eupenicillium alutaceum D.B. Scott, Mycopathol. Mycol. Appl. 36: 17 (1968). [MB330719]. - Herb.: CBS 317.67 p.p. (holotype). Ex-type cultures: CBS $317.67=$ ATCC $18542=$ FRR $1158=$ IFO $31728=$ IMI 136243. ITS barcode: AF033454. (Alternative markers: BenA = KJ834430; RPB2 = JN121489; CaM = KP016768).

Penicillium arabicum Baghd., Nov. Sist. Nizsh. Rast. 5: 105 (1968). [MB335711]. - Herb.: designated as T16 in Universitate Mosquensi (holotype); CBS H-7471 (isotype). Ex-type cultures: CBS $414.69=$ ATCC 22347 = DSM $2205=$ FRR $507=$ IMI $140335=$ VKM F-1077. 
ITS barcode: KC411758. (Alternative markersBenA = $\mathrm{KP} 016750 ; R P B 2=\mathrm{KP064574} ; \mathrm{CaM}=\mathrm{KP} 016770)$.

Penicillium atrolazulinum Visagie \& K. Jacobs. IMA Fungus 7: 91 (2016) [MB811001]. — Herb.: CBS H-22043 (holotype). Ex-type cultures: CBS 139136 = DAOMC 241083 = DTO 180-H4 = CV 55. ITS barcode: JX140913. (Alternative markers: BenA $=\mathrm{JX} 141077 ;$ RPB2 $=$ KP064575; CaM = JX157416).

Penicillium atrosanguineum B.X. Dong, Česká Mycol. 27: 174 (1973). [MB319260]. — Herb.: PRC 1397 (holotype), CBS H-15524 (isotype). Ex-type cultures: CBS 380.75 $=$ FRR 1726 = IMI 197488. ITS barcode: JN617706. (Alternative markers: BenA $=\mathrm{KJ} 834435 ; \quad \mathrm{RPB2}=$ JN406557; CaM = KP016771).

Penicillium bertai: See under Penicillium citreonigrum.

Penicillium burgense Quintan. ex Visagie. IMA Fungus 7: 94 (2016). [MB816641]. — Herb.: CBS H-22567 (dried specimen, holotype). Ex-type culture: CBS 325.89. ITS barcode: KC411736. (Alternative markers: BenA = $\mathrm{KJ} 834437 ;$ RPB2 = JN406572; CaM = KP016772).

Synonym: Penicillium burgense Quintan. Av. Aliment Majora Anim., 30: 176 (1990); nom. inval. (Art. 40.7) [MB130241]. - Herb.: Sáez 1538. Ex-type culture: CBS 325.89. ITS barcode: KC411736. (Alternative markers: BenA = $\mathrm{KJ} 834437 ;$ RPB2 = JN406572; CaM = KP016772).

Penicillium caerulescens: See under Penicillium raciborskii. Penicillium candidofulvum: See under Penicillium corylophilum.

Penicillium canis S.W. Peterson, J. Clin. Microbiol. 52: 2450 (2014). [MB807056]. - Herb.: BPI 892763 (holotype). Ex-type culture: NRRL 62798. ITS barcode: KJ511291. (Alternative markers: BenA $=\mathrm{KF900167;} \mathrm{RPB2=}$ KF900196; CaM = KF900177).

Penicillium catenatum D.B. Scott, Mycopathol. Mycol. Appl. 36: 24 (1968). [MB335719]. — Herb.: CBS 352.67 p.p. (holotype). Ex-type cultures: CBS $352.67=$ ATCC 18543 = CSIR 1097 = IFO $31774=$ IMI 136241. ITS barcode: KC411754. (Alternative markers: BenA = KJ834438; $R P B 2=\mathrm{JN} 121504 ;$ CaM = KP016774).

Synonym: Eupenicillium catenatum D.B. Scott, Mycopathol. Mycol. Appl. 36: 24 (1968). [MB330723]. - Herb.: CBS 352.67 p.p. (holotype). Ex-type cultures: CBS $352.67=$ ATCC 18543 = CSIR $1097=$ IFO $31774=$ IMI 136241. ITS barcode: KC411754. (Alternative markers: BenA = KJ834438; RPB2 = JN121504; CaM = KP016774).

Penicillium chalybeum: See under Penicillium terrenum.

Penicillium chloroleucon: See under Penicillium corylophilum

Penicillium chalabudae Visagie. IMA Fungus 7: 94 (2016). [MB816642]. - Herb.: CBS H-15439 (holotype). Ex-type cultures: CBS $219.66=$ ATCC $18322=$ ATCC $18329=$ FRR 3393 = VKM F-1037. ITS barcode: KP016811. (Alternative markers: BenA $=\mathrm{KP016748;}$ RPB2 = KP064572; CaM = KP016767).

Synonym: Penicillium albocinerascens Chalab., Not. Syst. Crypt. Inst. bot. Acad. Sci. USSR 6: 166 (1950); non (Maublanc) Biourge 1923; nom. illegit. (Art. 53.1). [MB302374]. - Herb.: CBS H-15439 (isotype). Ex-type cultures: CBS $219.66=$ ATCC $18322=$ ATCC $18329=$ FRR 3393 = VKM F-1037. ITS barcode: KP016811. (Alternative markers: BenA $=\mathrm{KP} 016748 ; \quad R P B 2=$ KP064572; CaM = KP016767).
Penicillium cinerascens Biourge, Cellule 33: 308 (1923). [MB260785]. - Herb.: K(M) IMI 92234 (neotype, Pitt et al. 2000). Ex-type cultures: NRRL $748=$ ATCC 48693 $=$ BIOURGE $90=$ FRR $748=$ IMI $92234=$ QM $7555=$ Thom 4733.34. ITS barcode: AF033455. (Alternative markers: $B e n A=\mathrm{JX141041;}$ RPB2 = KP064614; $\mathrm{CaM}=$ JX157405).

Penicillium cinereoatrum Chalab., Not. Syst. Crypt. Inst. bot. Acad. Sci. USSR. 6: 167 (1950). [MB302385]. - Herb.: CBS H-7469 (epitype designated here, MBT204705); loc. cit.: Fig. 7 -- lectotype designated here, MBT204706).. Ex-type cultures: CBS $222.66=$ NRRL3390 = ATCC $22350=$ FRR $3390=$ IJFM 5024 = IMI 113676 = VKM F-856. ITS barcode: KC411700. (Alternative markers: BenA $=\mathrm{KJ} 834442 ; \quad R P B 2=$ JN406608; CaM = KP125335).

Penicillium citreonigrum Dierckx, Ann. Soc. Sci. Bruxelles 25: 86 (1901). [MB165197]. — Herb.: K(M) IMI 92209i (neotype, Pitt 1980). Ex-type cultures: CBS $258.29=$ ATCC $48736=$ FRR $761=$ IMI $092209=$ LSHBP $20=$ LSHBP $98=$ MUCL $28648=$ MUCL $29062=$ MUCL $29116=$ NRRL 761. ITS barcode: AF033456. (Alternative markers: $B e n A=E F 198621 ; R P B 2=J N 121474 ; C a M=$ EF198628).

Synonyms: Penicillium subcinereum Westling, Ark. Bot. 11 (1): 137 (1911). [MB215824]. — Herb.: unknown. Extype cultures: CBS $258.29=$ ATCC $48736=$ FRR $761=$ IMI $092209=$ LSHBP $20=$ LSHBP $98=$ MUCL $28648=$ MUCL 29062 = MUCL $29116=$ NRRL 761. ITS barcode: AF033456. (Alternative markers: BenA = EF198621; $R P B 2=J N 121474 ; C a M=E F 198628)$. Note: Same type as Penicillium citreonigrum.

Penicillium citreoviride Biourge, Cellule 33: 297 (1923). [MB357253]. - Herb.: unknown. Ex-type cultures: CBS $308.48=$ ATCC $10425=$ FRR $2046=$ IMI $040575=$ NRRL 2046 (authentic according to Raper \& Thom 1949). ITS barcode: EF198647. (Alternative markers: BenA = EF198623; RPB2 = KP064616; CaM = KP016778).

Penicillium bertai Talice \& Mackinnon, Ann/s Parasit. Hum. Comp. 7: 97 (1929) (fide Pitt 1980). [MB258372]. Herb.: unknown. Ex-type culture: No culture available.

Penicillium citreoviride var. aeneum S. Abe, J. Gen. Appl. Microbiol. 2: 58 (1956); nom. inval. (Art. 39.1,). [MB123735]. - Herb.: unknown. Ex-type cultures: CBS $321.59=$ ATCC $18308=$ FAT123 = IFO $6224=$ IMI $068225=$ NRRL $3404=$ QM 7290. ITS barcode: KP016812. (Alternative markers: BenA = KP016749; RPB2 = KP064573; CaM = KP016769).

Penicillium aeneum G. Sm., Trans. Brit. Mycol. Soc. 46: 334 (1963). [MB335706]. - Herb.: IMI 68225 (holotype). Ex-type cultures: CBS $321.59=$ ATCC $18308=$ FAT123 = IFO $6224=$ IMI $068225=$ NRRL $3404=$ QM 7290. ITS barcode: KP016812. (Alternative markers: BenA = $\mathrm{KP} 016749 ;$ RPB2 = KP064573; CaM = KP016769).

Penicillium citreosulfuratum Biourge, Cellule 33: 285 (1923). [MB260947]. — Herb.: icon in Biourge (1923) Cellule 33: fig. 86 no 21 (lectotype); K(M) IMI 92228 (epitype). Ex-type cultures: IMI 92228 = DTO 290-14. ITS barcode: KP016814. (Alternative markers: BenA = $\mathrm{KP} 016753 ;$ RPB2 = KP064615; $C a M=\mathrm{KP} 016777)$. 
Synonym: Penicillium toxicarium I. Miyake, Rep. Res. Inst. Rice Improvement 1: 1 (1940); nom. inval. (Art. 39.1). [MB335772]. - Herb.: unknown. Ex-type culture: No culture available. ITS barcode: n.a. (Alternative markers: BenA = n.a.; $R P B 2=$ n.a.; $C a M=$ n.a.). Note: Non Penicillium toxicarium I. Miyake ex C. Ramírez, Man. Atlas Penicillia: 125 (1982) = Penicillium trzebinskii K.M. Zalessky, Bull. Int. Acad. Polon. Sci., sér. B., Sci. Nat. 1927: 498 (1927) (fide Houbraken et al. 2014). [MB280795]. - Herb.: unknown. Ex-type culture: CBS 351.51. Section Aspergilloides. ITS barcode: KM189449. (Alternative markers: BenA $=\mathrm{KM088673;} R P B 2=$ KM089445; CaM = KM089058).

Penicillium citreovirens: See under Penicillium corylophilum. Penicillium citreoviride: See under Penicillium citreonigrum.

Penicillium citreoviride var. aeneum: See under Penicillium citreonigrum.

Penicillium coeruleoviride: See under Penicillium corylophilum.

Penicillium consobrinum Visagie \& $\mathrm{K}$. Jacobs, IMA Fungus 7: 96 (2016). [MB811002]. - Herb.: CBS $\mathrm{H}-22045$ (holotype). Ex-type cultures: CBS $139144=$ DAOMC 241072 = DTO 181-H9 = CV547. ITS barcode: JX140888. (Alternative markers: BenA = JX141135; $R P B 2=\mathrm{KP064619;}$ CaM = JX157453).

Penicillium corylophilum Dierckx, Ann. Soc. Sci. Bruxelles 25: 86 (1901). [MB178294]. — Herb.: K(M) IMI 39754 (neotype, Pitt 1980). Ex-type cultures: CBS $312.48=$ TCC9784 = ATHUM2890 = CECT $2270=$ FRR 802 $=\mathrm{IMI} 039754=\mathrm{MUCL} 28671=\mathrm{MUCL} 29073=\mathrm{MUCL}$ $29131=$ NRRL $802=$ QM 7510. ITS barcode: AF033450. (Alternative markers: BenA = JX141042; RPB2 = KP064631; CaM = KP016780).

Synonyms: Penicillium candidofulvum Dierckx, Annls Soc. Scient. Brux. 25: 86, 1901. [MB175508]. - Herb.: unknown. Ex-type cultures: CBS $254.37=$ IMI $092201=$ LSHBP 12 = MUCL $28669=$ NRRL 769. ITS barcode: KC411712. (Alternative markers: BenA = KP016751; $R P B 2=$ KP064606; CaM = KP016773).

Penicillium chloroleucon Biourge, Cellule 33: 270 (1923). [MB260541]. - Herb.: unknown. Ex-type cultures: CBS $127808=$ IMI 92202. ITS barcode: KP016813. (Alternative markers: BenA = KP016752; RPB2 = KP064613; CaM = KP016776).

Penicillium coeruleoviride G. Sm., Trans. Brit. Mycol. Soc. 48: 275 (1965). [MB335720]. — Herb.: K(M) IMI 103148 (holotype). Ex-type cultures: CBS $259.67=$ ATCC 22351 $=$ FRR $3403=$ IFO $9146=$ IMI 103148. ITS barcode: KC411717. (Alternative markers: BenA = KP016754; RPB2 = KP064617; CaM = KP016779).

Penicillium humuli J.F.H. Beyma, Zentralbl. Bakteriol. Parasitenk., Abt.2 99: 393 (1939). [MB267441]. — Herb.: unknown. Ex-type cultures: CBS 231.38 = ATCC 10452 $=$ IFO $7726=$ IMI $039817=$ NRRL 872. ITS barcode: JN617696. (Alternative markers: BenA = KP016756; RPB2 = KP064645; CaM = KP016787).

Penicillium obscurum Biourge, Cellule 33: 267 (1923). [MB272613]. - Herb.: unknown. Ex-type cultures: CBS $127807=$ IMI $92263=$ NRRL $793=$ FRR 793. ITS barcode: KP016815. (Alternative markers: BenA = KP016761;
$R P B 2=\mathrm{KP} 064654 ; \mathrm{CaM}=\mathrm{KP}$ 016797).

Penicillium citreovirens S. Abe, J. Gen. Appl. Microbiol. 2: 87 (1956); nom. inval. (Art. 39.1). [MB302387]. — Herb.: unknown. Ex-type cultures: CBS $312.48=$ TCC9784 = ATHUM $2890=$ CECT $2270=$ FRR $802=$ IMI 039754 = MUCL 28671 = MUCL 29073 = MUCL 29131 = NRRL 802 = QM 7510. ITS barcode: GU944557. (Alternative markers: BenA = GU944519; RPB2 = JN406569; CaM = GU944607). Note: Same type as Penicillium corylophilum.

Penicillium corynephorum: See under Penicillium smithii.

Penicillium cravenianum Visagie \& K. Jacobs, IMA Fungus 7: 96 (2016). [MB811003]. - Herb.: CBS H-22044 (holotype). Ex-type cultures: CBS $139138=$ DAOMC 241082 = DTO 180-I5 = CV 92. ITS barcode: JX140900. (Alternative markers: BenA = JX141076; RPB2 = KP064636; CaM = JX157418).

Penicillium decumbens Thom, USDA Bur. Animal Industr. Bull. 118: 71 (1910). [MB156582]. - Herb.: K(M) IMI 190875 (neotype, Pitt 1980). Ex-type cultures: CBS $230.81=$ FRR $741=$ IMI $190875=$ MUCL $29107=$ NRRL 741. ITS barcode: AY157490. (Alternative markers: BenA = KJ834446; RPB2 = JN406601; CaM = KP016782).

Penicillium dimorphosporum H.J. Swart, Trans. Brit. Mycol. Soc. 55: 310 (1970). [MB120334]. - Herb.: CBS 456.70 (holotype). Ex-type cultures: CBS $456.70=$ NRRL $5207=$ ATCC $22783=$ ATCC $52501=$ FRR $1120=$ IMI 149680. ITS barcode: AF081804. (Alternative markers: BenA = KJ834448; RPB2 = JN121517; CaM = KP016783).

Penicillium dravuni Janso, Mycologia 97: 445 (2005). [MB501442]. - Herb.: BPI 844248 (holotype). Ex-type culture: F01V25. ITS barcode: AY494856. (Alternative markers: $B e n A=$ n.a.; RPB2 = n.a.; $C a M=$ n.a. $)$.

Penicillium erubescens D.B. Scott, Mycopathol. Mycol. Appl. 36: 14 (1968). [MB335726]. - Herb.: CBS 318.67 p.p. (holotype). Ex-type cultures: CBS $318.67=$ ATCC $18544=$ CSIR $1040=$ FRR $814=$ IFO $31734=I M I 136204$ = NRRL 6223. ITS barcode: AF033464. (Alternative markers: BenA = HQ646566; RPB2 = JN121490; CaM $=$ EU427281).

Synonym: Eupenicillium erubescens D.B. Scott, Mycopathol. Mycol. Appl. 36: 14 (1968). [MB330727]. - Herb.: CBS 318.67 p.p. (holotype). Ex-type cultures: CBS 318.67 $=$ ATCC $18544=$ CSIR $1040=$ FRR $814=$ IFO 31734 = IMI 136204 = NRRL 6223. ITS barcode: AF033464. (Alternative markers: BenA $=\mathrm{HQ646566;}$ RPB2 = JN121490; CaM = EU427281).

Penicillium fagi C. Ramírez \& A.T. Martínez, Mycopathologia 63: 57 (1978). [MB283595]. — Herb.: IJFM 3049 (holotype). Ex-type cultures: CBS 689.77 = CCMF-696 $=$ IJFM $3049=$ IMI 253806 = VKMF-2178. ITS barcode: AF481124. (Alternative markers: BenA = KJ834449; $R P B 2=\mathrm{JN} 406540 ; \mathrm{CaM}=\mathrm{KP} 016784)$.

Penicillium flavidostipitatum: See under Penicillium namyslowskii.

Penicillium gilmanii: See under Penicillium restrictum.

Penicillium griseolum G. Sm., Trans. Br. Mycol. Soc. 40: 485 (1957). [MB302401]. - Herb.: LSHTM BB 323 (holotype), K(M) IMI 071626 (isotype). Ex-type cultures: CBS $277.58=$ ATCC $18239=$ FRR $2671=$ IFO 8175 
= IMI $071626=$ LSHBB323 = NRRL $2671=$ QM 7523. ITS barcode: EF422848. (Alternative markers: BenA = EF506213; RPB2 = KP064638; CaM = EF506232).

Penicillium griseum: See under Penicillium restrictum.

Penicillium guttulosum J.C. Gilman \& E.V. Abbott, lowa St. Coll. J. Sci. 1: 298 (1927). [MB266689]. - Herb.: CBS $\mathrm{H}-22566$ (epitype designated here), MBT204703; loc. cit.: Fig. 33 - lectotype designated here, MBT204704). Ex-type cultures: CBS $141171=$ NRRL $907=$ ATCC $48734=$ FRR $907=$ Thom 4894.16. ITS barcode: HQ646592. (Alternative markers: BenA = HQ646576; $R P B 2=\mathrm{KP064639;} C a M=\mathrm{HQ646587})$.

Penicillium hemitrachum Visagie \& K. Jacobs, IMA Fungus 7: 99 (2016). [MB811004]. - Herb.: CBS H-22042 (holotype). Ex-type cultures: CBS $139134=$ DAOMC $241098=$ DTO 180-D8 = CV 2845. ITS barcode: FJ231003. (Alternative markers: BenA = JX141048; $R P B 2=\mathrm{KP064642} ; \mathrm{CaM}=\mathrm{JX} 157526)$.

Penicillium heteromorphum H.Z. Kong \& Z.T. Qi, Mycosystema 1: 107 (1988). [MB135444]. — Herb.: CBS 226.89 (neotype, Frisvad et al. 1990). Ex-type culture: CBS 226.89. ITS barcode: KC411702. (Alternative markers: BenA = KJ834455; RPB2 = JN406605; CaM = KP016786).

Penicillium humuli: See under Penicillium corylophilum.

Penicillium katangense Stolk, Antonie van Leeuwenhoek 34: 42 (1968). [MB120725]. - Herb.: CBS 247.67 p.p. (holotype). Ex-type cultures: CBS 247.67 = ATCC 18388 $=$ IMI $136206=$ NRRL 5182. ITS barcode: AF033458. (Alternative markers: BenA $=\mathrm{KP} 016757 ; \mathrm{RPB2}=$ KP064646; CaM = KP016788).

Synonym: Eupenicillium katangense Stolk, Antonie van Leeuwenhoek 34: 42 (1968). [MB330732]. — Herb.: CBS 247.67 p.p. (holotype). Ex-type cultures: CBS $247.67=$ ATCC $18388=$ IMI $136206=$ NRRL 5182. ITS barcode: AF033458. (Alternative markers: BenA = KP016757; $R P B 2=\mathrm{KP064646} ; \mathrm{CaM}=\mathrm{KP} 016788)$.

Penicillium kurssanovii Chalab., Bot. Mater. Otd. Sporov. Rast. 6: 168 (1950). [MB274327]. — Herb.: unknown. Extype cultures: CBS $625.67=$ ATCC $18387=$ FRR 3381 = IJFM 5045 = IMI 129965 = NRRL 3381 = VKMF-1244. ITS barcode: EF422849. (Alternative markers: BenA = $\mathrm{KP} 016758 ; R P B 2=\mathrm{KP} 064647 ; \mathrm{CaM}=\mathrm{KP} 016789)$.

Penicillium laeve (K. Ando \& Manoch) Houbraken \& Samson, Stud. Mycol. 70: 47 (2011). [MB561960]. Herb.: TNS-F-238517 (holotype). Ex-type cultures: CBS $136665=\mathrm{KY} 12727=$ NBRC 109724. ITS barcode: KF667369. (Alternative markers: BenA = KF667365; $R P B 2=\mathrm{KF667371} ; \mathrm{CaM}=\mathrm{KF667367})$.

Synonym: Torulomyces laevis K. Ando \& Manoch, Mycoscience 39: 317 (1998). [MB447110].

Penicillium lapidosum Raper \& Fennell, Mycologia 40: 524 (1948). [MB289094]. - Herb.: K(M) IMI 39743 p.p. (holotype). Ex-type cultures: CBS 343.48 = ATCC 10462 = CCT4477 = IFO $6100=$ IMI $039743=$ NRRL $718=$ QM 1928. ITS barcode: AF033409. (Alternative markers: BenA = KJ834465; RPB2 = JN121500; CaM = FJ530984).

Synonym: Eupenicillium lapidosum D.B. Scott \& Stolk, Antonie van Leeuwenhoek 33: 298 (1967). [MB330733]. - Herb.: K(M) IMI 39743 p.p. (holotype). Ex-type cultures: CBS $343.48=$ ATCC $10462=$ CCT4477 = IFO $6100=$ IMI $039743=$ NRRL $718=$ QM 1928. ITS barcode: AF033409. (Alternative markers: BenA = KJ834465; RPB2 = JN121500; CaM = FJ530984).

Penicillium luzoniacum: See under Penicillium terrenum.

Penicillium maclennaniae H.Y. Yip, Trans. Brit. Mycol. Soc. 77: 202 (1981). [MB112523]. — Herb.: DAR 35238 (holotype). Ex-type cultures: CBS 198.81 = DAR 35238. ITS barcode: KC411689. (Alternative markers: BenA = KJ834468; RPB2 = KP064648; CaM = KP016791).

Penicillium melinii Thom, Penicillia: 273(1930). [MB270876]. - Herb.: K(M) IMI 40216 (neotype, Pitt 1980). Ex-type cultures: CBS $218.30=$ ATCC $10469=$ FRR $2041=$ IFO 7675 = IMI 040216 = MUCL 29235 = NRRL $2041=$ QM 7599. ITS barcode: AF033449. (Alternative markers: BenA = KJ834471; RPB2 = JN406613; CaM = KP016792).

Synonym: Penicillium radulatum G. Sm., Trans. Brit. Mycol. Soc. 40: 484 (1957). [MB302420]. - Herb.: LSHTM BB 333 (holotype). Ex-type cultures: CBS 280.58 = ATCC $18383=$ IMI $071624=$ LSHBB333 = NRRL $2672=\mathrm{QM}$ 7526. ITS barcode: KC411727. (Alternative markers: BenA = KP016763; RPB2 = KP064658; CaM = KP016801).

Penicillium menonorum S.W. Peterson, IMA Fungus 2: 122 (2011). [MB519297]. - Herb.: BPI 881018 (holotype). Ex-type culture: NRRL 50410. ITS barcode: HQ646591. (Alternative markers: BenA $=\mathrm{HQ646573;}$ RPB2 = KF900194; CaM = HQ646584).

Penicillium meridianum D.B. Scott, Mycopathol. Mycol. Appl. 36: 12 (1968). [MB335750]. — Herb.: CBS 314.67 p.p. (holotype). Ex-type cultures: CBS $314.67=$ ATCC $18545=$ CSIR $1052=$ IMI 136209. ITS barcode: AF033451. (Alternative markers: BenA = KJ834472; RPB2 = JN406576; CaM = KP016794).

Synonym: Eupenicillium meridianum D.B. Scott, Mycopathol. Mycol. Appl. 36: 12 (1968). [MB330736]. — Herb.: CBS 314.67 p.p. (holotype). Ex-type cultures: CBS $314.67=$ ATCC $18545=$ CSIR $1052=$ IMI 136209. ITS barcode: AF033451. (Alternative markers: BenA = KJ834472; $R P B 2=\mathrm{JN} 406576 ;$ CaM $=\mathrm{KP016794})$.

Penicillium momoii Visagie \& K. Jacobs, IMA Fungus 7: 99 (2016). [MB811007]. — Herb.: CBS H-22046 (holotype). Ex-type cultures: CBS $139157=$ DAOMC $241077=$ DTO 182-G4 = CV 1015. ITS barcode: JX140895. (Alternative markers: BenA = JX141073; RPB2 = KP064673; CaM = JX157479).

Penicillium namyslowskii K.M. Zalessky, Bull. Int. Acad. Polonc. Sci., Cl. Sci. Math., sér. B, Sci. Nat. 1927: 479 (1927). [MB272006]. — Herb.: CBS 353.48 (neotype, Pitt 1979). Ex-type cultures: CBS $353.48=$ ATCC $11127=$ IMI 040033 = MUCL 29226 = NRRL 1070. ITS barcode: AF033463. (Alternative markers: BenA = JX141067; RPB2 = JF417430; CaM = KP016795).

Synonyms: Geosmithia namyslowskii (K.M. Zalessky) Pitt, Can. J. Bot. 57: 2024 (1979). [MB314414].

Penicillium flavidostipitatum C. Ramírez \& C.C. González, Mycopathologia 88: 3 (1984). [MB106338]. — Herb.: CBS 202.87 (neotype, Frisvad et al. 1990). Ex-type cultures: CBS 202.87 = IJFM 7824. ITS barcode: KC411691. (Alternative markers: BenA $=\mathrm{KJ} 834451 ; \mathrm{RPB2}=$ KP064637; CaM = KP016785). 
Penicillium turris-painense C. Ramírez, Mycopathologia 91: 93 (1985). [MB274385]. - Herb.: unknown. Ex-type culture: CBS 204.87. ITS barcode: KC411693. (Alternative markers: BenA = KP016766; RPB2 = KP064681; CaM = KP016809).

Penicillium nepalense Takada \& Udagawa, Trans. Mycol. Soc. Japan 24: 146 (1983). [MB108327]. - Herb.: NHL 6482 p.p. (holotype). Ex-type cultures: CBS 203.84 $=\mathrm{NHL}$ 6482. ITS barcode: KC411692. (Alternative markers: $B$ enA $=\mathrm{KJ} 834474 ; R P B 2=\mathrm{JN} 121453 ;$ CaM $=$ KP016796).

Synonym: Eupenicillium nepalense Takada \& Udagawa, Trans. Mycol. Soc. Japan 24: 146 (1983). [MB107965]. Herb.: NHL 6482 p.p. (holotype). Ex-type cultures: CBS 203.84 = NHL 6482. ITS barcode: KC411692. (Alternative markers: BenA = KJ834474; RPB2 = JN121453; CaM = KP016796).

Penicillium obscurum: See under Penicillium corylophilum.

Penicillium ovatum (K. Ando \& Nawawi) Houbraken \& Samson, Stud. Mycol. 70: 48 (2011). [MB561961]. Herb.: TNS-F-238518 (holotype). Ex-type cultures: CBS $136664=\mathrm{KY} 12726$. ITS barcode: KF667370. (Alternative markers: BenA = KF667366; RPB2 = KF667372; CaM = KF667368).

Synonym: Torulomyces ovatus K. Ando \& Nawawi, Mycoscience 39: 317 (1998). [MB447111]. - Herb.: TNS-F-238518 (holotype). Ex-type cultures: CBS 136664 $=\mathrm{KY}$ 12726. ITS barcode: KF667370. (Alternative markers: BenA = KF667366; RPB2 = KF667372; CaM = KF667368).

Penicillium pagulum Visagie \& K. Jacobs, IMA Fungus 7: 102 (2016). [MB811005]. — Herb.: CBS H-22049 (holotype). Ex-type cultures: CBS $139166=$ DAOMC $241069=$ DTO 183-H2 = CV 2224. ITS barcode: JX140898. (Alternative markers: BenA = JX141070; RPB2 = KP064655; CaM = JX157519).

Penicillium papuanum: See under Penicillium parvum.

Penicillium parvum Raper \& Fennell, Mycologia 40: 508 (1948). [MB289101]. — Herb.: CBS 359.48 p.p. (holotype). Ex-type cultures: CBS $359.48=$ ATCC 10479 = IFO 7732 = IMI $040587=$ NRRL $2095=$ QM 1878. ITS barcode: AF033460. (Alternative markers: BenA = HQ646568; RPB2 = JN406559; CaM = KF900173).

Synonyms: Eupenicillium parvum (Raper \& Fennell) Stolk \& D.B. Scott, Persoonia 4: 402 (1967). [MB330739].

Eupenicillium papuanum Udagawa \& Y. Horie, Trans. Mycol. Soc. Japan 14: 378 (1973). [MB313983]. - Herb.: NHL 6463 p.p. (holotype). Ex-type cultures: CBS $570.73=$ ATCC $28050=$ ATCC $48363=$ FRR $1559=$ NHL 6463. ITS barcode: KC411767. (Alternative markers: BenA = KP016762; RPB2 = KP064657; CaM = KP016798).

Penicillium papuanum Udagawa \& Y. Horie, Trans. Mycol. Soc. Japan 14: 378 (1973). [MB319290]. — Herb.: NHL 6463 p.p. (holotype). Ex-type cultures: CBS $570.73=$ ATCC $28050=$ ATCC $48363=$ FRR $1559=$ NHL 6463. ITS barcode: KC411767. (Alternative markers: BenA = KP016762; RPB2 = KP064657; CaM = KP016798).

Penicillium philippinense Udagawa \& Y. Horie, J. Jap. Bot. 47: 341 (1972). [MB319291]. - Herb.: NHL 6130 p.p. (holotype). Ex-type cultures: CBS $623.72=\mathrm{FRR}$
$1532=\mathrm{NHL}$ 6130. ITS barcode: KC411770. (Alternative markers: BenA = KJ834482; RPB2 = JN406543; CaM = KP016799).

Synonym: Eupenicillium philippinense Udagawa \& Y. Horie, J. Jap. Bot. 47: 341 (1972). [MB313984]. — Herb.: NHL 6130 p.p. (holotype). Ex-type cultures: CBS $623.72=$ FRR $1532=$ NHL 6130. ITS barcode: KC411770. (Alternative markers: $B$ enA = KJ834482; RPB2 = JN406543; CaM = KP016799).

Penicillium pimiteouiense S.W. Peterson, Mycologia 91: 271 (1999). [MB460126]. - Herb.: BPI 806262 (holotype). Ex-type cultures: CBS $102479=$ NRRL 25542. ITS barcode: AF037431. (Alternative markers: BenA = $\mathrm{HQ646569;}$ RPB2 = JN406650; CaM = HQ646580).

Penicillium raciborskii K.M. Zalessky, Bull. Int. Acad. Polon. Sci., sér. B, Sci. Nat. 1927: 454 (1927). [MB276002]. Herb.: K(M) IMI 40568 (neotype, Pitt et al. 2000). Ex-type cultures: CBS 224.28 = ATCC $10488=$ DSM2422 = FRR $2150=$ IFO $7676=$ IMI $040568=$ LSHBP $92=\mathrm{MUCL}$ $29246=$ NRRL 2150. ITS barcode: AF033447. (Alternative markers: $B e n A=\mathrm{JX} 141069 ; \mathrm{RPB2}=\mathrm{JN} 406607 ; \mathrm{CaM}=$ KP016800).

Synonym: Penicillium caerulescens Quintan., Mycopathologia 82: 101 (1983) (fide Frisvad et al. 1990). [MB108323]. - Herb.: unknown. Ex-type culture: Q1147 (personal collection of Quintanilla). ITS barcode: n.a. (Alternative markers: $B e n A=$ n.a.; RPB2 = n.a.; $C a M=$ n.a.).

Penicillium radulatum: See under Penicillium melinii.

Penicillium repensicola Visagie \& $\mathrm{K}$. Jacobs, IMA Fungus 7: 102 (2016). [MB811006]. — Herb.: CBS H-22047 (holotype). Ex-type cultures: CBS $139160=$ DAOMC $241080=$ DTO 183-B8 = CV 1495. ITS barcode: JX140893. (Alternative markers: BenA = JX141150; $R P B 2=\mathrm{KP064660} ;$ CaM $=\mathrm{JX157490)}$.

Penicillium restrictum J.C. Gilman \& E.V. Abbott, lowa St. Coll. J. Sci. 1: 297 (1927). [MB276289]. — Herb.: K(M) IMI 40228 (neotype, Pitt 1980). Ex-type cultures: CBS $367.48=$ ATCC $11257=$ FRR $1748=$ IMI $040228=$ NRRL $1748=$ QM 1962. ITS barcode: AF033457. (Alternative markers: $B e n A=\mathrm{KJ} 834486 ; R P B 2=\mathrm{JN} 121506 ; \mathrm{CaM}=$ KP016803).

Synonyms: Penicillium gilmanii Thom, Penicillia: 345 (1930) (fide Pitt 1980). [MB265991]. - Herb.: unknown. Ex-type culture: No culture available.

Penicillium griseum (Sopp) Biourge, Cellule 33: 103 (1923) (fide Pitt 1980). [MB152791]. — Herb.: unknown. Ex-type culture: No culture available.

Citromyces griseus Sopp, Skr. VidenskSelsk. Christiana 11: 119 (1912). [MB491119].

Penicillium rubefaciens Quintan., Mycopathologia 80: 73 (1982). [MB109998]. - Herb.: CBS 145.83 (neotype, Frisvad et al. 1990). Ex-type cultures: CBS 145.83 = CECT 2752. ITS barcode: KC411677. (Alternative markers: BenA $=\mathrm{KJ} 834487 ;$ RPB2 $=\mathrm{JN} 406627 ;$ CaM $=$ KP016804).

Penicillium rubidurum Udagawa \& Y. Horie, Trans. Mycol. Soc. Japan 14: 381 (1973). [MB319295]. — Herb.: NHL 6460 p.p. (holotype). Ex-type cultures: CBS $609.73=$ NRRL $6033=$ ATCC $28051=$ ATCC $48238=$ FRR 1558 $=$ IMI $228551=$ NHL 6460. ITS barcode: AF033462. 
(Alternative markers: BenA $=\mathrm{HQ646574;}$ RPB2 = JN406545; CaM = HQ646585).

Synonym: Eupenicillium rubidurum Udagawa \& Y. Horie, Trans. Mycol. Soc. Japan 14: 381 (1973). [MB313985]. - Herb.: NHL 6460 p.p. (holotype). Ex-type cultures: CBS $609.73=$ NRRL $6033=$ ATCC $28051=$ ATCC 48238 $=$ FRR $1558=$ IMI $228551=$ NHL 6460. ITS barcode: AF033462. (Alternative markers: BenA = HQ646574; $R P B 2=\mathrm{JN} 406545 ;$ CaM $=\mathrm{HQ646585})$.

Penicillium sabulosum: See under Penicillium smithii.

Penicillium smithii Quintan., Av. Aliment. Majora Anim. 23: 340 (1982). [MB114173]. - Herb.: CBS 276.83 (neotype, Pitt et al. 2000). Ex-type cultures : CBS 276.83 = CECT 2744 = IMI 259693. ITS barcode: KC411723. (Alternative markers: BenA = KJ834492; RPB2 = JN406589; CaM = KP016806).

Synonyms: Penicillium corynephorum Pitt \& A. D. Hocking, Mycotaxon 22: 202 (1985). [MB105609]. - Herb.: FRR 2663 (holotype). Ex-type cultures: CBS 256.87 = FRR $2663=$ IMI 288724. ITS barcode: KC411716. (Alternative markers: BenA $=\mathrm{KP} 016755 ;$ RPB2 $=\mathrm{KP} 064635 ; \mathrm{CaM}=$ KP016781).

Penicillium sabulosum Pitt \& A. D. Hocking, Mycologia 77: 818 (1985). [MB104604]. — Herb.: FRR 2743 (holotype). Ex-type cultures: CBS 261.87 = FRR 2743. ITS barcode: KC411719. (Alternative markers: BenA = KP016765; $R P B 2=\mathrm{KP} 064672 ; C a M=\mathrm{KP} 016805)$.

Talaromyces sabulosus (Pitt \& A.D. Hocking) Samson, Yilmaz \& Frisvad, Stud. Mycol. 70: 177 (2011). [MB560673].

Penicillium striatisporum Stolk, Antonie van Leeuwenhoek 35: 268 (1969). [MB335769]. - Herb.: CBS 705.68 (holotype). Ex-type cultures: CBS 705.68 = ATCC 22052 = CCRC $31679=$ FRR $827=$ IMI 151749 = MUCL 31202. ITS barcode: AF038938. (Alternative markers: BenA = JX141156; RPB2 = JN406538; CaM = KP016807).

Penicillium subcinereum: See under Penicillium citreonigrum.

Penicillium subturcoseum Visagie \& $\mathrm{K}$. Jacobs, IMA Fungus 7: 105 (2016). [MB811008]. - Herb.: CBS $\mathrm{H}-22041$ (holotype). Ex-type cultures: CBS $139132=$ DAOMC 241096 = DTO 180-C9 = CV 2835. ITS barcode: FJ231006. (Alternative markers: BenA = JX141161; $R P B 2=\mathrm{KP} 064674 ; C a M=\mathrm{JX} 157532)$.

Penicillium terrenum D.B. Scott, Mycopathol. Mycol. Appl. 36: 1 (1968). [MB335771]. — Herb.: CBS 313.67 p.p. (holotype). Ex-type cultures: CBS $313.67=$ ATCC 18547 = CSIR 1022 = IMI 136208. ITS barcode: AM992111. (Alternative markers: BenA $=\mathrm{KJ} 834496 ; \quad R P B 2=$ JN406577; CaM = KP016808).

Synonyms: Eupenicillium terrenum D.B. Scott, Mycopathol. Mycol. Appl. 36: 1 (1968). [MB330745]. — Herb.: CBS 313.67 p.p. (holotype). Ex-type cultures: CBS $313.67=$ ATCC $18547=$ CSIR $1022=$ IMI 136208. ITS barcode: AM992111. (Alternative markers: BenA = KJ834496; $R P B 2=\mathrm{JN} 406577 ;$ CaM $=\mathrm{KP} 016808)$.

Penicillium luzoniacum Udagawa \& Y. Horie, J. Jap. Bot. 47: 338 (1972). [MB319284]. — Herb.: CBS H-7120 p.p. (isotype). Ex-type cultures: CBS $622.72=\mathrm{DSM} 2418$ $=$ NHL $6128=$ NRRL 5824. ITS barcode: AF033446. (Alternative markers: BenA $=\mathrm{KP016759;}$ RPB2 = JN406544; CaM = KP016790).
Eupenicillium luzoniacum Udagawa \& Y. Horie, J. Jap. Bot. 47: 338 (1972). [MB313979]. — Herb.: CBS H-7120 p.p. (isotype). Ex-type cultures: CBS $622.72=$ DSM2418 $=\mathrm{NHL} 6128=\mathrm{NRRL}$ 5824. ITS barcode: AF033446. (Alternative markers: BenA = KP016759; RPB2 = JN406544; CaM = KP016790).

Penicillium chalybeum Pitt \& A.D. Hocking, Mycotaxon 22: 204 (1985). [MB105608]. - Herb.: FRR 2660 (holotype). Ex-type cultures: CBS $254.87=$ FRR 2660. ITS barcode: KC411713. (Alternative markers: BenA = KJ834440; RPB2 = JN406596; CaM = KP016775).

Penicillium toxicarium: See under Penicillium citreosulfuratum.

Penicillium turris-painense: See under Penicillium namyslowskii.

Penicillium velutinum J.F.H. Beyma, Zentralbl. Bakteriol. Parasitenk., Abt. 2 91: 353 (1935). [MB283175]. - Herb.: K(M) IMI 40571 (neotype, Pitt 1980). Ex-type cultures: CBS $250.32=$ ATCC $10510=$ CECT $2318=$ IJFM 5108 = IMI 040571 = NRRL $2069=$ QM 7686 = VKMF-379. ITS barcode: AF033448. (Alternative markers: BenA = JX141170; RPB2 = KP064682; CaM = KP016810).

Penicillium vinaceum J.C. Gilman \& E.V. Abbott, lowa St. Coll. J. Sci. 1: 299 (1927). [MB281754]. — Herb.: K(M) IMI 29189 (neotype, Pitt 1980). Ex-type cultures: CBS $389.48=$ ATCC $10514=$ FRR $739=$ IMI $029189=$ NRRL 739 = QM 6746. ITS barcode: AF033461. (Alternative markers: BenA $=\mathrm{HQ646575} ; \mathrm{RPB2}=\mathrm{JN} 406555 ; \mathrm{CaM}$ = HQ646586).

Penicillium xanthomelinii Visagie \& $\mathrm{K}$. Jacobs, IMA Fungus 7: 105 (2016). [MB811009]. - Herb.: CBS $\mathrm{H}-22048$ (holotype). Ex-type cultures: CBS $139163=$ DAOMC 241104 = DTO 183-C7 = CV 1677. ITS barcode: JX140921. (Alternative markers: BenA = JX141120; RPB2 = KP064683; CaM = JX157495).

Talaromyces sabulosus: See under Penicillium smithii.

Torulomyces laevis: See under Penicillium leave.

Torulomyces ovatus: See under Penicillium ovatum.

\section{DISCUSSION}

Penicillium sect. Exilicaulis is a taxonomically diverse group currently containing 83 Penicillium species names, including 11 Eupenicillium basionyms and one variety. Using four genes and applying GCPSR, we accept 43 of these species and introduce nine new species isolated from the fynbos biome in South Africa. The difficulty of distinguishing some species in section Exilicaulis using morphology alone is clear in the $P$. corylophilum-clade, where some complexes differ only inconspicuously whereas some neighbouring phylogenetically coherent species display considerable phenotypic variation. For example, we introduce six new species closely related to $P$. rubefaciens, but there are only minor morphological differences between some of these, although they are all phylogenetically distinct; i.e. they are "cryptic species". The contrary example is $P$. atrolazulinum, which from a morphological perspective might represent multiple species (Fig. 7), but phylogenetically is only one (Figs 3-4). This again emphasizes the importance of sequence-based identifications and species circumscriptions in Penicillium, a 
genus now containing 406 accepted species (Visagie et al. $2014 b$ ), making it one of the larger fungal genera. This number is ever increasing with 41 species introduced since the end of 2014 (Perrone et al. 2015, Peterson et al. 2015, Rong et al. 2015, Visagie et al. 2015, 2016a, b, You et al. 2014). At least five new fynbos species remain undescribed adding to the already 27 described during our fynbos diversity project. From experience we know that the fynbos biome still has a huge reservoir of undescribed species, especially when we start exploring other regions and more microhabitats similar to that of Protea repens. It would not be surprising if the number of new species described from this unique South African floral region reaches a century. The fynbos, however, represents a small proportion of the world inventory and it begs the question as to how many species remain undescribed from the world's other 24 biodiversity hotspots (Myers et al. 2000). Species estimates is a controversial subject but the general consensus for fungi is a conservative 1.5 million (Hawksworth 1991, 2001). To make such an estimate for Penicillium is of course very much speculative, as we simply do not have the type of dataset that Hawksworth $(1991,2001)$ had (i.e. plant:fungi ratio). With that being said, the total number of Penicillium may well reach close to 1000 when considering the possibility of cryptic species, the number of new species that we know will be described in the next few years and the many unexplored or under-sampled habitats in the world. We therefore again emphasize how important it is that new descriptions be accompanied by sequence data of preferably the ITS and BenA regions as a minimum requirement.

The ITS is the accepted DNA barcode for fungi (Schoch et al. 2014), but contains insufficient variation to distinguish several species of sect. Exilicaulis. This is also true for other groups of Penicillium and resulted in Visagie et al. (2014b) proposing BenA as a secondary identification marker for the genus. This gene was selected as it is easy to sequence, reference data is openly available and mostly complete, and most importantly works well for species identifications across the genus. In section Exilicaulis, ITS distinguishes species in the $P$. citreonigrum-, $P$. decumbens-, and $P$. parvumclades, but performs poorly elsewhere. In comparison, BenA distinguishes all species in the section and is sufficient for identification purposes. CaM would work equally as well, but for RPB2 we experienced difficulties amplifying and sequencing, and internal primers were often needed to obtain clean sequences. These genes are, however, useful for applying GCPSR to delineate species, and thus can be recommended for widespread use in taxonomic studies of Penicillium.

Correct identification of Penicillium sect. Exilicaulis species is important because they have important roles in a wide range of habitats. Penicillium corylophilum was recently reported as a common indoor contaminant (McMullin et al. 2014, Visagie et al. 2014a), where it can produce several extrolites that may cause allergies in humans (Kremer et al. 1989, McMullin et al. 2014, Ohnishi et al. 2002, Unoura et al. 2011). Penicillium pimiteouiense and $P$. canis were respectively isolated from polycystic kidney cell structures (Peterson et al. 1999) and a dog suffering with osteomyelitis (Langlois et al. 2014), and could be significant to animal health. Another medical example is $P$. decumbens, commonly reported from indoor air (Samson et al. 2010), which was isolated from a para-vertebral infection (Lyratzopoulos et al. 2002) and was reported as the causative agent of a fungus ball (Yoshida et al. 1992).

Penicillium citreosulfuratum (as "P. toxicarium") was closely associated with cork bark (Quercus suber) in Portugal (Serra et al. 2008), and although not considered a health concern, may contribute to mouldy flavours in wine. Penicillium citreosulfuratum ("P. toxicarium" in Serra et al.) and $P$. citreonigrum (syn. $P$. citreoviride) are good producers of citreoviridin (Peterson et al. 2015), a mycotoxin linked to yellow rice disease or acute cardiac beri-beri in Japan (Miyake 1940, Sakabe et al. 1977, Udagawa \& Tatsuno 2004, Uraguchi 1969) and more recently in Brazil (Almeida et al. 2012, Rosa et al. 2010). Citreoviridin production is associated with numerous species from various substrates (Cole et al. 1981, Wicklow \& Cole 1984, Wicklow et al. 1988, Wigmann et al. 2015); Peterson et al. (2015) updated identifications from previous studies, confirming that many Penicillium species do produce citreoviridin.

We consider $P$. citreosulfuratum the correct name for $P$. toxicarium. It is unknown whether the ex-type culture of $P$. citreosulfuratum (IMI 92228) produces citreoviridin. Penicillium cinerascens has $P$. citreonigrum and $P$. citreosulfuratum as its closest relatives and does not produce citreoviridin. The description of $P$. toxicarium by Miyake (1940) was invalid because he did not include a Latin diagnosis. Ramírez (1982) later validated the name based on an ex-type strain CBS 351.51 , noting that the culture had changed noticeably from the original description; that strain was evidently a contaminant and did not represent the species observed by Miyake. Houbraken et al. (2014) showed that sequences obtained from CBS 351.51 are identical to $P$. trzebinskii in section Aspergilloides. Serra et al. (2008) identified a number of isolates from cork bark $(Q$. suber) collected in Portugal as $P$. toxicarium and obtained sequences showing a close relationship to $P$. citreonigrum. Since then, numerous sequences have been deposited in GenBank as $P$. toxicarium based on that application of the name. Unfortunately, Serra et al. (2008) did not epitypify the name or indicate which strain they considered to be the ex-type. Although we agree that this clade possibly represents Miyake's (1940) original species, our data show that $P$. citreosulfuratum is the correct and older name for the clade, and we reduce $P$. toxicarium to a synonym. Adopting this name also resolves the confusion surrounding the application of $P$. toxicarium.

Several species accepted here were previously considered synonyms of other species, including $P$. arabicum, $P$. albocinerascens and $P$. kurssanovii, all part of the $P$. restrictum-clade. Although the section represents a species complex, these three species have unique sequences and are thus considered distinct. Unfortunately, no ex-type cultures are available for $P$. bertai $(=P$. citreonigrum), $P$. gilmanii $(=P$. restrictum) and $P$. griseum $(=P$. restrictum), and we thus accept the synonymies proposed by Pitt (1980). Penicillium glaucolanosum was considered synonymous with $P$. decumbens (Pitt 1980), but does not belong in section Exilicaulis and is probably a synonym of $P$. janthinellum (Houbraken \& Samson 2011). 
The ecology of Penicillium species inside Protea repens infructescences is intriguing. After a flowering season, usually between fall and winter, Protea repens inflorescences close and become woody (serotinous) and then only mites and insects can enter or exit. Penicillium atrolazulinum and $P$. citreosulfuratum had a close association with Protea repens infructescences and their mite communities. Reasons for this dominance are unclear but seem unlikely to be a coincidence because they dominated isolations from three very distinct collection sites, during three different flowering seasons. Perhaps there is an unknown selective pressure favouring their growth inside these infructescences, or mites could perhaps carry them into flowers where they start to outcompete other species for available nutrients when the flower closes. A mutualistic association between mites and a fungus in Protea infructescences was previously reported for Ophiostoma, but no association was found with any Penicillium species (Roets et al. 2007). During isolations from living mites, we observed that they commonly fed on colonies from these two Penicillium species. In Petri dishes, they would almost exclusively lay their eggs in $P$. citreosulfuratum colonies. The relationships among the flowers, mites, and the two Penicillium species should be investigated further to address this apparent feeding preference. Similar behaviour has been noted for other penicillia: Kobayashi et al. (2008) and Li et al. (2012) found $P$. herquei to almost exclusively colonize leaf rolls of Euops chinensis, with Li et al. (2012) suggesting a mutualistic relationship based on the fungus significantly improving development of larvae. Similarly, $P$. coffeae was described as an associate of the coffee-berry borer (Hypothenemus hampei) with speculation that the fungus might provide the beetle with the exogenous sterols necessary for development (Peterson et al. 2003). That mites preferred to feed and lay eggs in $P$. citreosulfutatum colonies suggests a similar mutualism. One hypothesis is that the fungus provides necessary nutrients to mites, while the mites act as vectors of dispersal to and from enclosed Protea repens infructescences. With regard to species distribution, it would be interesting to investigate whether $P$. citreosulfuratum: (1) is present in other Protea species; (2) is present in Protea farmed for export; and (3) includes strains capable of producing citreoviridin.

\section{ACKNOWLEDGEMENTS}

We acknowledge the South African Biosystematics Initiative (SABI) of the National Research Foundation (NRF) for financial support during this project. This project was also partly funded by the Alfred P. Sloan Foundation Program on the Microbiology of the Built Environment, which supported C.M.V. during his time at CBS-KNAW where the work for this manuscript was finalised. The Western Cape Nature Conservation Board issued permits for collecting soil and Protea repens infructescences in the fynbos. Hugh Glen is acknowledged for providing assistance on the new scientific names used in the manuscript. We are also grateful to Scott A. Redhead for providing assistance on typification and nomenclatural issues addressed in this paper.

\section{REFERENCES}

Almeida MI, Almeida NG, Carvalho KL, Goncalves GA, Silva CN, Santos EA, Garcia JC, Vargas EA (2012) Co-occurrence of aflatoxins $B(1), B(2), G(1)$ and $G(2)$, ochratoxin $A$, zearalenone, deoxynivalenol, and citreoviridin in rice in Brazil. Food Additives \& Contaminants, A 29: 694-703.

Chalabuda TV (1950) Species novae e genere Penicillium Link. Notulae systematicae e Sectione Cryptogamica Instituti Botanici nomine VL Komarovii Academiae Scientiarum URSS Botanicheskie materialy 6: 161-169

Cole RJ, Dorner JW, Cox RH, Hill RA, Cluter HG, Wells JM (1981) Isolation of citreoviridin from Penicillium charlesii cultures and molded pecan fragments. Applied and Environmental Microbiology 42: 677-681.

Frisvad JC (1981) Physiological criteria and mycotoxin production as aids in identification of common asymmetric penicillia. Applied and Environmental Microbiology 41: 568-579.

Frisvad JC, Samson RA, Stolk AC (1990) Disposition of recently described species of Penicillium. Persoonia 14: 209-232.

Gouy M, Guindon S, Gascuel O (2010) SeaView Version 4: A multiplatform graphical user interface for sequence alignment and phylogenetic tree building. Molecular Biology and Evolution 27: 221-224.

Hawksworth DL (1991) The fungal dimension of biodiversity: magnitude, significance, and conservation. Mycological Research 95: 641-655.

Hawksworth DL (2001) The magnitude of fungal diversity: the 1.5 million species estimate revisited. Mycological Research 105: 1422-1432.

Houbraken J, Frisvad JC, Samson RA (2011) Taxonomy of Penicillium section Citrina. Studies in Mycology 70: 53-138.

Houbraken J, Samson RA (2011) Phylogeny of Penicillium and the segregation of Trichocomaceae into three families. Studies in Mycology 70: 1-51.

Houbraken J, Visagie CM, Meijer M, Frisvad JC, Busby PE, et al. (2014) A taxonomic and phylogenetic revision of Penicillium section Aspergilloides. Studies in Mycology 78: 373-451.

Katoh K, Standley DM (2013) MAFFT multiple sequence alignment software version 7: improvements in performance and usability. Molecular Biology and Evolution 30: 772-780.

Kobayashi C, Fukasawa Y, Hirose D, Kato M (2008) Contribution of symbiotic mycangial fungi to larval nutrition of a leaf-rolling weevil. Evolutionary Ecology 22: 711-722.

Kornerup A, Wanscher JH (1967) Methuen Handbook of Colour. $2^{\text {nd }}$ edn. London: Methuen.

Knapp A (2014) Find Momo. $1^{\text {st }}$ edn. Philadelphia: Quirk Books.

Kremer AM, Pal TM, de Monchy JGR, Kauffman HF, de Vries K (1989) Precipitating antibodies and positive skin tests in workers exposed to airborne antigens from a contaminated humidification system. International Archives of Occupational and Environmental Health 61: 547-553.

Langlois DK, Sutton DA, Swenson CL, Bailey CJ, Wiederhold NP, et al. (2014) Clinical, morphological, and molecular characterization of Penicillium canis sp. nov., isolated from a dog with osteomyelitis. Journal of Clinical Microbiology 52: 2447-2453.

Li X, Guo W, Ding J (2012) Mycangial fungus benefits the development of a leaf-rolling weevil, Euops chinesis. Journal of Insect Physiology 58: 867-873. 
Lyratzopoulos G, Ellis M, Nerringer R, Denning DW (2002) Invasive Infection due to Penicillium species other than $P$. marneffei. Journal of Infection 45: 184-195.

Martinez A, Ramírez C (1978) Penicillium fagi sp. nov., isolated from beech leaves. Mycopathologia 63: 57-59.

McMullin DR, Nsiama TK, Miller JD (2014) Secondary metabolites from Penicillium corylophilum isolated from damp buildings. Mycologia 106: 621-628.

Miyake I (1940) Studies on toxin production by a saprophyte growing on stored rice. Report of the Rice Utilization Laboratories, Hôkoku, Japan 1: 1-30. [In Japanese.]

Myers N, Mittermeier RA, Mittermeier CG, Fonseca GAB da, Kent $\mathrm{J}(2000)$ Biodiversity hotspots for conservation priorities. Nature 403: 853-858.

Nylander AJJ, Ronquist F, Huelsenbeck JP, Nieves-Aldrey JL (2004) Bayesian phylogenetic analysis of combined data. Systematic Biology 53: 47-67.

Ohnishi T, Yamada G, Tanaka H, Nakajima K, Tanaka S, et al. (2002) A case of chronic hypersensitivity pneumonia with elevation of serum SP-D and KL-6. Nihon Kokyuki Gakkai Zasshi 40: 66-70.

Perrone G, Samson RA, Frisvad JC, et al. (2015) Penicillium salamii, a new species occurring during seasoning of dry-cured meat. International Journal of Food Microbiology 193: 91-98

Peterson SW, Corneli S, Hjelle J, Miller-Hjelle M, Nowak D, Bonneau $P$ (1999) Penicillium pimiteouiense: A new species isolated from polycystic kidney cell cultures. Mycologia 91: 269-277.

Peterson SW, Jurjević Ž, Frisvad JC (2015) Expanding the species and chemical diversity of Penicillium section Cinnamopurpurea. PloS One 10: e0121987.

Peterson SW, Orchard SS, Menon S (2011) Penicillium menonorum, a new species related to $P$. pimiteouiense. IMA Fungus 2: 121125.

Peterson SW, Pérez J, Vega F, Infante F (2003) Penicillium brocae, a new species associated with the coffee berry borer in Chiapas, Mexico. Mycologia 95: 141-147.

Peterson SW, Jurjević Ž, Frisvad JC (2015) Expanding the species and chemical diversity of Penicillium section Cinnamopurpurea. PloS One 10: e0121987.

Pitt JI (1979) Geosmithia gen. nov. for Penicillium lavendulum and related species. Canadian Journal of Botany 57: 2021-2030.

Pitt JI (1980) ["1979"] The Genus Penicillium and its Teleomorphic States Eupenicillium and Talaromyces. London: Acdemic Press.

Pitt JI, Samson RA, Frisvad JC (2000) List of accepted species and their synonyms in the family Trichocomaceae. In: Integration of Modern Taxonomic Methods for Penicillium and Aspergillus. (Samson RA, Pitt JI, eds): 9-79. Amsterdam: Harwood Academic Press.

Quintanilla JA (1982) Three new species of Penicillium isolated from soil. Mycopathologia 80: 73-82.

Quintanilla JA (1990) Penicillium cluniae nov. sp. y P. burgense nov. sp., dos nuevas especies aisladas de suelo no cultivado. Avances en Alimentation y Mechora Animal 30: 174-180

Ramírez C (1982) Manual and Atlas of the Penicillia. Amsterdam: Elsevier Biomedical Press.

Rivera KG, Seifert KA (2011) A taxonomic and phylogenetic revision of the Penicillium sclerotiorum complex. Studies in Mycology $\mathbf{7 0}$ : 139-158.

Roets F, Wingfield MJ, Crous PW, Dreyer LL (2007) Discovery of fungus-mite mutualism in a unique niche. Environmental Entomology 36: 1226-1237.
Ronquist F, Huelsenbeck JP (2003) MrBayes 3: Bayesian phylogenetic inference under mixed models. Bioinformatics 19: 1572-1574.

Rosa CAR, Keller KM, Oliveira AA, Almeida TX, Keller LAM, et al. (2010) Production of citreoviridin by Penicillium citreonigrum strains associated with rice consumption and beriberi cases in the Maranhão State, Brazil. Food Additives and Contaminants 27: 241-248.

Sakabe N, Goto T, Hirata Y (1977) Structure of citreoviridin, a mycotoxin produced by Penicillium citreo-viride molded on rice. Tetrahedron 33: 3077-3081.

Samson RA, Houbraken J, Thrane U, Frisvad JC, Andersen B (2010) Food and Indoor Fungi. [CBS Laboratory Manual no. 2.] Utrecht: CBS-KNAW Fungal Biodiversity Centre.

Schoch CL, Robbertse B, Robert V, et al. (2014) Finding needles in haystacks: linking scientific names, reference specimens and molecular data for Fungi. Database (Oxford) 2014: DOI:10.1093/ database/bau061.

Serra R, Peterson S, Venancio A (2008) Multilocus sequence identification of Penicillium species in cork bark during plank preparation for the manufacture of stoppers. Research in Microbiology 159: 178-186.

Tamura K, Stecher G, Peterson D, Filipski A, Kumar S (2013) MEGA6: Molecular Evolutionary Genetics Analysis version 6.0. Molecular Biology and Evolution 30: 2725-2729.

Taylor JW, Jacobson DJ, Kroken S, Kasuga T, Geiser DM, Hibbett DS, Fisher MC (2000) Phylogenetic species recognition and species concepts in fungi. Fungal Genetics and Biology 31: 21-32.

Udagawa S, Tatsuno T (2004) Safety of rice grains and mycotoxins - a historical review of yellow rice mycotoxicoses. Yakushigaku Zasshi 39: 321-42.

Unoura K, Miyazaki Y, Sumi Y, Tamaoka M, Sugita T, Inase N (2011) Identification of fungal DNA in BALF from patients with homerelated hypersensitivity pneumonitis. Respiratory Medicine 105: $1696-1703$.

Uraguchi K (1969) Mycotoxin origin of cardiac Beriberi. Journal of Stored Products Research 5: 227-236.

Visagie CM, Hirooka Y, Tanney JB, Whitfield E, Mwange K, et al. (2014a) Aspergillus, Penicillium and Talaromyces isolated from house dust samples collected around the world. Studies in Mycology 78: 63-139.

Visagie CM, Houbraken J, Dijksterhuis J, Seifert KA, Jacobs K, Samson RA (2016a) A taxonomic review of Penicillium species producing conidiophores with solitary phialides, classified in section Torulomyces. Persoonia 36: 134-155.

Visagie CM, Houbraken J, Frisvad JC, Hong S-B, Klaassen CHW, et al. (2014b) Identification and nomenclature of the genus Penicillium. Studies in Mycology 78: 343-371.

Visagie CM, Houbraken J, Rodriques C, Pereira CS, Dijksterhuis J, et al. (2013) Five new Penicillium species in section Sclerotiora: a tribute to the Dutch Royal family. Persoonia 31: 42-62.

Visagie CM, Houbraken J, Seifert KA, Samson RA, Jacobs K (2015) Four new Penicillium species isolated from the fynbos biome in South Africa, including a multigene phylogeny of section LanataDivaricata. Mycological Progress 14: 486-502.

Visagie CM, Renaud JB, Burgess KMN, et al. (2016b) Fifteen new species of Penicillium. Persoonia 36: 247-280.

Visagie CM, Seifert KA, Houbraken J, Samson RA, Jacobs K (2014c) Diversity of Penicillium section Citrina within the fynbos biome of South Africa, including a new species from a Protea repens 
infructescence. Mycologia 106: 537-552.

Wicklow DT, Cole RJ (1984) Citreoviridin in standing corn infested by Eupenicillium ochrasalmoneum. Mycologia 76: 959-961.

Wicklow DT, Stubblefield RD, Horn BW, Shotwell OL (1988) Citreoviridin levels in Eupenicillium ochrosalmoneuminfested maize kernels at harvest. Applied and Environmental Microbiology 54: 1096-1098.

Wigmann ÉF, Saccomori F, Bernardi AO, Frisvad JC, Copetti MV (2015) Toxigenic penicillia spoiling frozen chicken nuggets. Food Research International 67: 219-222.

Rong C, Ma Y, Wang S, et al. (2016) Penicillium chroogomphum, a new species in Penicillium section Ramosa isolated from fruiting bodies of Chroogomphus rutilus in China. Mycoscience 57: 79-84.
Yip HY (1981) Penicillium maclennaniae sp. nov. Transactions of the British Mycological Society 77: 202-203.

Yoshida K, Hiraoka T, Ando M, Uchida K, Mohsenin V (1992) Penicillium decumbens: a new cause of fungus ball. Chest 101: 1152-1153.

You Y-H, Cho H-S, Song J, et al. (2014) Penicillium koreense sp. nov., isolated from various soils in Korea. Journal of Microbiology and Biotechnology 24: 1606-1608. 